\title{
The Interplanetary and Magnetospheric causes of Geomagnetically Induced Currents (GICs) > 10 A in the Mäntsälä Finland Pipeline: 1999 through 2019
}

\author{
Bruce T. Tsurutani ${ }^{1, *}$ and Rajkumar Hajra ${ }^{2}$ \\ ${ }^{1}$ Jet Propulsion Laboratory, California Institute of Technology, Pasadena, California 91109, USA \\ 2 Indian Institute of Technology Indore, Simrol, Indore 453552, India
}

Received 9 October 2020 / Accepted 7 January 2021

\begin{abstract}
The interplanetary and magnetospheric phenomena time-coincident with intense geomagnetically induced current (GIC) > 10 A and > 30 A events during 21 years (1999 through 2019) at the Mäntsälä, Finland (57.9 $9^{\circ}$ magnetic latitude) gas pipeline have been studied. Although forward shocks and substorms are predominant causes of intense GICs, some newly discovered geoeffective interplanetary features are: solar wind plasma parcel (PP) impingements, possible interplanetary magnetic field (IMF) northward (Bn) and southward (Bs) turnings, and reverse shocks. The PPs are possibly the loop and filament portions of coronal mass ejections (CMEs).

From a study of $>30$ A GIC events, it is found that supersubstorm (SSS: SML $<-2500 \mathrm{nT}$ ) and intense substorm $(-2500 \mathrm{nT}<S M L<-2000 \mathrm{nT})$ auroral electrojet intensifications are the most frequent $(76 \%)$ cause of all of these GIC events. These events occur most often $(76 \%)$ in superstorm $(S Y M-H \leq-250 \mathrm{nT})$ main phases, but they can occur in other storm phases and lesser intensity storms as well. After substorms, PPs were the most frequent causes of Mäntsälä GIC > 30 A events. Forward shocks were the third most frequent cause of the $>30$ A events. Shock-related GICs were observed to occur at all local times.

The two "Halloween" superstorms of 29-30 and 30-31 October 2003 produced by far the greatest number of GICs in the interval of study ( $9>30$ A GICs and $168>10$ A GICs). In the first Halloween superstorm, a shock-triggered SSS (SML <-3548 nT) caused 33, 57, 51 and 52 A GICs. The 57 A GIC was the most intense event of the superstorm and of this study. It is possible that this SSS is a new form of substorm. Equally intense magnetic storms were also studied but their related GICs were far less numerous and less intense.
\end{abstract}

Keywords: GIC / Supersubstorm / Plasma Parcel / Shock / ICME / Superstorm

\section{Introduction}

Geomagnetically induced currents (GICs) are currents induced in the solid Earth or in conductors on the Earth's surface by sudden, intense currents flowing in space plasmas. Such a phenomenon was noted a century and a half ago when the deflection of telegraph magnetic needles of the Midland Railroad (England) was observed to be coincident with auroral sightings (Barlow, 1849). Currents were noted to flow even when the batteries were not connected. More recently Campbell (1980) observed electric currents flowing in the Alaska oil pipeline and deduced that the source was the auroral electrojet current (a midnight sector current flowing in the ionosphere at $\sim 100 \mathrm{~km}$ altitude with intensities of up to and possibly greater than $\left.10^{6} \mathrm{~A}\right)$.

This paper was motivated by the extensive GIC data set existing for the natural gas pipeline near Mäntsälä, Finland $\left(57.9^{\circ} \mathrm{N}\right.$ geomagnetic latitude; $60.6^{\circ} \mathrm{N}$ geographic latitude, $25.2^{\circ} \mathrm{E}$ geographic longitude: Pirjola \& Lehtinen, 1985; Pulkkinen et al., 2001; Viljanen et al., 2006). Viljanen et al. (2010) performed a nice 11-year study on this data set for GIC > 5 A events during magnetic storm intervals. The purpose of this present paper is to identify for the first time the interplanetary, magnetospheric and ionospheric phenomena time-coincident with intense

*Corresponding author: bruce.tsurutani@gmail.com

\section{JSWSC}

10 years anniversary issue
Special Issue - 10 years of JSWSC 
> 10 A and > 30 A Mäntsälä GIC events over a 21-year interval, from 1 January 1999 to 31 December 2019.

This paper is intended to be a top-level survey, to identify the possible relationship between Mäntsälä intense GIC events and interplanetary time-coincident features. We will also identify the Mäntsälä GIC relationships with magnetic storms and substorms (which are causes by interplanetary magnetic field (IMF) southward component Bs) to obtain information about the relationship between the intensity of the GICs and the intensities of the magnetospheric and ionospheric activity.

We caution the reader that Mäntsälä is located at subauroral (57.9 $9^{\circ}$ magnetic) latitudes, so the interplanetary and magnetospheric causes of GICs at auroral latitudes, mid-latitudes, and equatorial latitudes might be different. Similar studies on other data sets will be needed to determine the causes of GICs at these other regions of the Earth.

Why are we performing this study? All GIC events on Earth are believed to be ultimately related to interplanetary drivers. The $\mathrm{d} B / \mathrm{d} t$ events that cause Mäntsälä GICs could also cause GICs in other subauroral systems. Of course, the GIC intensities for the other subauroral systems will be different (owing to different physical layouts, ground conductances, etc.), but statistically the physical interplanetary causes may be the same. A survey of this type has never been performed before. It will be shown that new, important potential causes of GICs at Mäntsälä will be discovered.

\section{Databases, methods of analyses, and related background space plasma observations}

\subsection{GIC data}

This study was undertaken using the current intensity measurements taken from the natural gas pipeline near Mäntsälä, Finland $\left(57.9^{\circ} \mathrm{N}\right.$ geomagnetic). See Pulkkinen et al. (2001) and Viljanen et al. (2010) for more details. Viljanen et al. (2010) have indicated that the Mäntsälä GIC measurement noise level is about $1 \mathrm{~A}$. The measurements were taken at $10 \mathrm{~s}$ intervals and for this study, we examine only the largest events with intensities of $10 \mathrm{~A}$ (and also $30 \mathrm{~A}$ ) current or greater. The study covers an interval of time from 1 January 1999 to 30 December 2019, almost two full solar cycles. Event intervals with GICs larger than $10 \mathrm{~A}$ (and $30 \mathrm{~A}$ ) are recorded and are discussed in the Appendix. All of the GIC event data have interplanetary and geomagnetic activity data superposed, and are shown as figures in the Appendix. The GIC data are available from the Space and Earth Observation Centre of the Finnish Meteorological Institute (https://space.fmi.fi/gic/index.php). The solar wind and geomagnetic activity data are discussed separately, below.

Figure 1 shows the Mäntsälä GIC data availability over the study interval as a function of solar activity cycle phase. The top panel is the solar F10.7 radiation intensity in solar flux units (sfu) as a function of year (bottom panel, horizontal axis), indicating the phase of the solar cycle. 1999 is in the ascending phase of solar cycle 23 (SC23) and 2019 is in the minimum phase between SC24 and SC25. The bottom panel shows the Mäntsälä data availability with the universal time (UT) given as the vertical axis. Smoothing was performed to give a general indication for

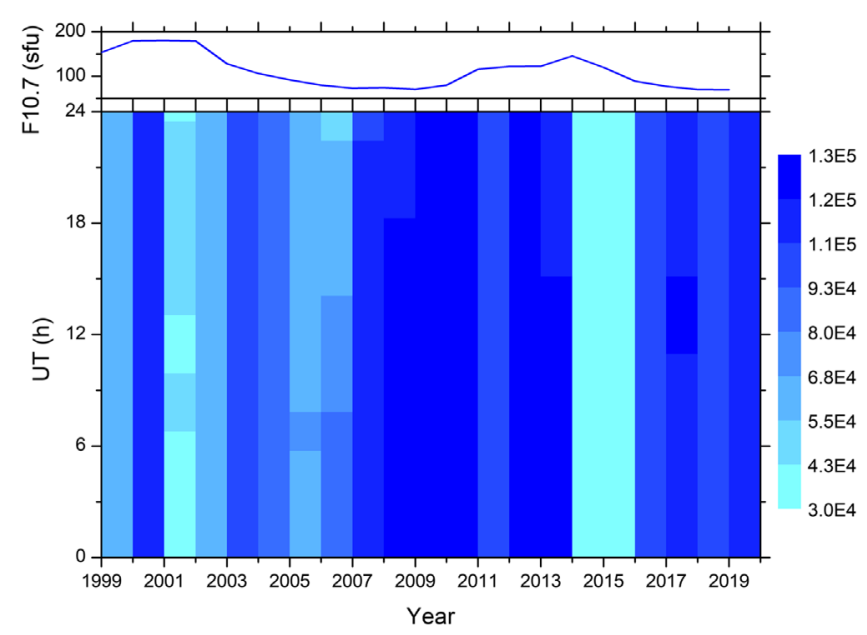

Fig. 1. The GIC data coverage for this study. The top panel gives the F10.7 solar flux in solar flux unit (sfu), where a sfu is $10^{-22} \mathrm{~W} \mathrm{~m}^{-2} \mathrm{~Hz}^{-1}$. The bottom panel gives the universal time (UT) of Mäntsälä in the vertical axis and year in the horizontal axis. The legend for the lower panel color figure on the right shows the numbers of $10 \mathrm{~s}$ GIC observations during each UT-year sector.

the reader. The legend is given on the right. It can be noticed that during the year 2014-2015 where the coverage was "low", there were still over 30,000 $10 \mathrm{~s}$ GIC data intervals available. The top coverage occurred between 2007-2013 and 2016-2019 with over 100,000 $10 \mathrm{~s}$ intervals at all UTs. The difference between year 2014 and 2015 and the maximum coverage is only a factor of 3 times. We conclude that the GIC data coverage at Mäntsälä over the interval of study was quite good.

For our study, Mäntsälä GIC data was available for $128,506 \mathrm{~h}$ out of $184,080 \mathrm{~h}$ possible (21 years), or $69.8 \%$ of the time. We have used cutoff intensities of $>10 \mathrm{~A}$ and $>30 \mathrm{~A}$ for this present study. There were $605>10 \mathrm{~A}$ and $21>30$ A GIC events found in this study.

The Mäntsälä GICs were noted to develop with time. GIC events could be as short as $20 \mathrm{~s}$ and as long as $13 \mathrm{~min}$. The average duration was $\sim 2 \mathrm{~min}$. In this paper we plot the event at the time of the peak intensity, rounded to the nearest minute.

\subsection{Solar wind data}

The solar wind and IMF data (1 min time resolution) were obtained from the OMNI website (https://omniweb.gsfc.nasa. gov/). The solar wind data for the 29-30 October and 30-31 October 2003 "Halloween storms" were obtained from R. Skoug and were used in the Mannucci et al. (2005) paper. The magnetic field components are given in geocentric solar magnetospheric (GSM) coordinates.

\subsection{Geomagnetic activity data: magnetic storms}

The geomagnetic $S Y M-H$ and Dst indices were obtained from the World Data Center for Geomagnetism, Kyoto, Japan (http://wdc.kugi.kyoto-u.ac.jp/). The $1 \mathrm{~min}$ average $S Y M-H$ indices and the $1 \mathrm{~h} D s t$ indices will be used to identify magnetic storm occurrences and intensities.

Magnetic storms are identified by the criteria $S Y M-H \leq$ -50 nT (Gonzalez et al., 1994). For each magnetic storm that occurred related to Mäntsälä GIC events > $10 \mathrm{~A}$, the peak storm 
intensity (to the nearest nT) will be given. In addition, we will identify superstorms with $S Y M-H \leq-250$ nT (Tsurutani et al., 1992; Gonzalez et al., 2007; Echer et al., 2008a; Meng et al., 2019) to indicate a higher-level cutoff of storm intensity. Superstorms will be shown to play a major role with the production of GICs.

The causes of magnetic storms have been well established to be due to the process of magnetic reconnection between southward IMFs (Bs) and the northward magnetic fields of the Earth's dayside magnetopause (Dungey, 1961) and the formation of a magnetospheric ring current (Williams, 1985; Daglis et al., 1999). This relationship between the IMF Bs and magnetic storms has been well documented in the literature (e.g., Gonzalez \& Tsurutani, 1987; Tsurutani et al., 1988; Gonzalez et al., 1989, 2007; Zhang et al., 2007; Echer et al., 2008b; Meng et al., 2019) and will be noted in the examination of magnetic storm events.

IMF northward (Bn) turnings cause cessation of ongoing geomagnetic activity and lead to geomagnetic quiet conditions (Tsurutani \& Gonzalez, 1995; Du et al., 2008). Several of the latter cases will be noted in the results of this paper.

\subsection{Geomagnetic activity data: substorms}

The geomagnetic $A L$ and $A U$ indices were obtained from the World Data Center for Geomagnetism, Kyoto, Japan (http:// wdc.kugi.kyoto-u.ac.jp/). The $1 \mathrm{~min}$ average, $\sim 12$ station $A L$ and $A U$ indices are located near the auroral oval and are best used to identify substorms during relatively low to moderate level geomagnetic activity.

The 1 min average $S M L$ and $S M U$ indices were taken from the SuperMAG network (http://supermag.jhuapl.edu/). These indices are based on $~ 300$ ground-based magnetometers and give much finer spatial scale resolution plus subauroral latitudinal coverage. The $S M L$ and $S M U$ indices are particularly useful during magnetic storms when auroras occur at locations below the auroral oval (at midlatitudes).

In this paper the peak $S M L$ intensity of pertinent substorms will be noted. In addition, substorms will be sorted into regular "substorms" ( $-50 \mathrm{nT}>S M L>-2000 \mathrm{nT})$, "large substorms" $(-2000 \mathrm{nT}>S M L>-2500 \mathrm{nT})$ and "supersubstorms" (SSSs: $S M L<-2500 \mathrm{nT}$, Tsurutani et al., 2015) to give a general sorting of substorm intensities.

Isolated substorms, those that occur outside of magnetic storms have been shown to be caused by IMF Bs intervals (Tsurutani \& Meng, 1972; Meng et al., 1973). The IMF Bs turnings led to substorm onsets and subsequent IMF Bn turnings led to substorm terminations. What is the difference between IMF Bs for substorms and the much larger magnetic storms? The IMF Bs for isolated substorms were of a shorter-duration ( $\sim 30$ min to $\sim 1 \mathrm{~h}$ compared to $\sim 1 \mathrm{~h}$ to $\sim 3 \mathrm{~h}$ ) and of lower intensities ( $\sim 5$ to $\sim 10 \mathrm{nT}$ in comparison to $\sim 20$ to $\sim 100 \mathrm{nT}$ ).

\subsection{Interplanetary shocks}

Interplanetary fast forward shocks ("shocks") will be identified by their abrupt increases in solar wind velocity (Vsw), density (Nsw), temperature (Tsw), and IMF magnitude (Bo). Interplanetary fast reverse shocks are noted by their abrupt increases in Vsw and simultaneous decreases in Nsw, Tsw and Bo (Kennel et al., 1985; Stone \& Tsurutani, 1985; Tsurutani \& Stone, 1985; Tsurutani et al., 2011). To identify the properties of the shocks (quasi-parallel: Kennel et al., 1984a, b or quasiperpendicular, Mach number, ram pressure), detailed high resolution upstream and downstream plasma and magnetic field properties must be used to identify the shock normal direction relative to the upstream magnetic field and the RankineHugoniot relations to obtain the shock Mach number. These detailed calculations will be foregone for the present. In this study we will tentatively identify shocks by their jump conditions alone. A more detailed study of shocks and their GIC effects will be postponed for a second, follow-up study. Why is it necessary to eventually identify shocks using the RankineHugoniot conservation equations? One objective is to separate shocks from other features such as waves and tangential discontinuities (TDs). Possible density changes across TDs or compressive waves can also produce magnetospheric compressions or rarefactions.

Compression of the magnetosphere/magnetotail by interplanetary shocks can cause the triggering of substorms in the nightside magnetosphere. These triggered substorms can be particularly intense (Hajra \& Tsurutani, 2018). For this reason, in the text that follows and in the Appendix, we have given the UT of the shocks. The local time (LT) of Finland is UT $+3 \mathrm{~h}$. Thus, one can determine the LT of Mäntsälä when the shock impingement occurred on the magnetosphere. At this time we do not know whether the shock created the GIC or whether the sharp onset of the substorm triggered by the shock caused the GIC. This topic is delayed for a future study.

\subsection{Interplanetary PPs (High-Plasma Densities: ICME loops? and filaments?)}

Interplanetary high plasma density features of the plasma parcels (PPs) impacting the magnetosphere/magnetotail will have the same effect as interplanetary shocks. They will compress the magnetosphere and magnetotail.

\subsection{Interplanetary sheaths}

The sheath upstream of an interplanetary coronal mass ejection (ICME) is composed of slow solar wind plasma and magnetic fields that have been compressed, heated and swept up by the shock (Tsurutani et al., 1988). This sheath plasma and magnetic fields are therefore totally different than the ICME plasma and magnetic fields. The sheath will be referred to separately in this paper. We have also identified geoeffective solar wind events that do not have shocks. We have called these upstream regions "pileup" events without examining them in further detail here.

\subsection{CMEs and their parts (loops, MCs and filaments)}

A CME at the Sun has three parts: a bright outer loop, a dark region and a filament (Illing \& Hundhausen, 1986; Chen, 2011). We distinguish interplanetary CMEs (ICMEs) from solar CMEs because not all three parts of a solar CME reach the Earth. In this study we have identified primarily ICME magnetic clouds (MCs; Burlaga et al., 1981) as being geoeffective and causing magnetic storms. Farrugia et al. (1997) and Tsurutani \& Gonzalez (1997) have argued that the MC portion of an ICME is the dark region of a CME detected near the Sun. In this study we use the criterion of low plasma $\beta$ (the ratio of the plasma thermal pressure to the magnetic pressure) to identify MCs. 
Another feature of ICMEs at $1 \mathrm{AU}$ are high density loops and filaments. The bright outer loop plasma and magnetic field data were identified at 1 AU by Tsurutani \& Gonzalez (1995) and Tsurutani et al. (1998a). ICME filament high plasma densities were first identified in interplanetary data at $1 \mathrm{AU}$ by Burlaga et al. (1998). It is possible that the high-density PPs identified in this study causing GICs are loops and filaments.

\section{Results}

Figure 2 shows the solar wind parameters, IMF and geomagnetic activity indices in the top 11 panels. The Mäntsälä GIC data is shown in the bottom panel. The parameters given in the various panels are identified in the figure caption. There is a shock at $\sim 0612$ UT on day 302 indicated by the vertical black dashed line.

The $S Y M-H$ index shows that this was a double dip main phase superstorm with a peak intensity of $-390 \mathrm{nT}$ at $\sim 0148$ UT on day 303. The partial cause of the first dip in the magnetic storm was the negative $\mathrm{Bz}$ (IMF Bs) just prior to and just after the shock (see blue trace). That and the energetic plasma injection due to the SSS (note the intense negative $S M L$ and $A L$ at the shock; to be discussed below) triggered by the shock caused the first dip storm. This magnetic storm (and the SSS) occurred during the interplanetary sheath region of the event, from the shock at $\sim 0612$ UT until $~ 0938$ UT. After the termination of the sheath, a MC follows. The MC ends at 0244 UT on day 303. The MC Bz component is first positive (IMF Bn) and then negative (IMF Bs), consistent with a giant magnetic flux rope (Burlaga et al., 1981). The IMF Bs of the MC starts at $\sim 1436$ UT on day 302 and ends at 0210 UT on day 303. The IMF Bs causes the second and major dip of the magnetic storm.

The shock occurred at the same time as a GIC peak of $25 \mathrm{~A}$. Mäntsälä was at 0912 LT (morning sector) at the time of the event. The shock also triggered a sharp onset of a two-peak SSS that lasted from $\sim 0613$ UT to $\sim 0752$ UT. The two peaks had intensities of $S M L=-3177 \mathrm{nT}$ at $0624 \mathrm{UT}$ and $-3548 \mathrm{nT}$ at $0648 \mathrm{UT}$, respectively. Associated with the first SSS were 4 GICs of intensities $>30 \mathrm{~A}$. The $30 \mathrm{~A}$ level is indicated by a horizontal red line. The GIC intensities and times were: $33 \mathrm{~A}$ at $0646 \mathrm{UT}, 57 \mathrm{~A}$ at $0657 \mathrm{UT}, 51 \mathrm{~A}$ at $0703 \mathrm{UT}$ and $52 \mathrm{~A}$ at 0727 UT. The SSS peaks occurred when SYM-H was $+13 \mathrm{nT}$ (the $S Y M-H$ index was not pressure corrected).

Note that enhanced GIC activity $(<10$ A) is present throughout the entire $\sim 21 \mathrm{~h}$ of the superstorm. Strong GIC activity extends from the beginning of the storm main phase to the start of the storm recovery phase. There are 168 GIC events with intensities $>10 \mathrm{~A}$ in the two main phases of this double dip storm. However, the most intense GIC events occurred in the first SSS immediately after the shock.

We point out several more GICs with intensities $>30 \mathrm{~A}$. There is a GIC of $30 \mathrm{~A}$ at 2230 UT (0130 LT) near the maximum of the second dip of the superstorm. This GIC is associated with an intense substorm of $S M L=-2340 \mathrm{nT}$. There is a $36 \mathrm{~A}$ GIC at 0110 UT (0410 LT) on day 303 at the start of the second dip storm recovery phase. There is no obvious substorm relationship for that event.

The 29-30 October 2003 Halloween superstorm had the greatest number of GICs $>10 \mathrm{~A}(168)$ in the 21-year data

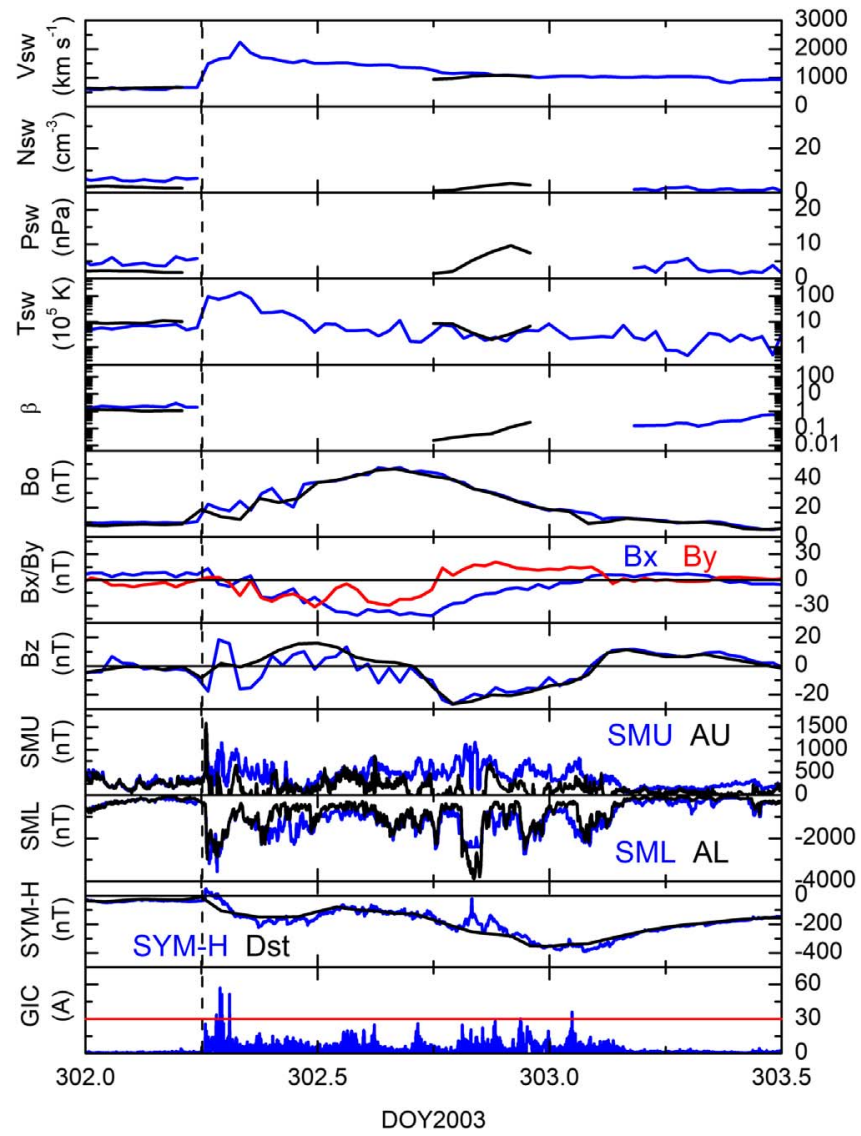

Fig. 2. The first "Halloween storm", days 302-303 (29-30 October), 2003. From the top panel downward are the solar wind velocity (Vsw), density (Nsw), ram pressure (Psw), temperature (Tsw), plasma $\beta$, magnetic field magnitude (Bo), the $B x$ and $B y$ components (in the same panel) and $B z$ in GSM coordinates. The bottom four panels are the $S M U$ and $A U$ indices (in the same panel), the $S M L$ and $A L$ indices (in the same panel), the $S Y M-H$ and Dst indices (in the same panel) and the GIC intensities. In the solar wind/interplanetary and geomagnetic indices panels, blue and black curves show $1 \mathrm{~min}$ and $1 \mathrm{~h}$ resolution data, respectively. The $x$-axis shows UT in the unit of day of year (DOY). The horizontal red line in the GIC panel indicates the GIC $=30 \mathrm{~A}$ intensity level. A vertical black dashed line indicates the time of an interplanetary shock.

study. These two days of a superstorm also had the greatest number of GICs $>30$ A (6) in the study. The 57 A GIC at 0657 UT on day 302 was the most intense GIC detected in this study.

Figure 3 shows the second Halloween magnetic storm on days 303-304 of year 2003. A sheath Bs upstream of an ICME causes a superstorm of intensity $S Y M-H=-432 \mathrm{nT}$. A solar wind density spike (PP) at $\sim 1949 \mathrm{UT}$ (2249 LT) caused a $\mathrm{SI}^{+}$of $61 \mathrm{nT}$ and triggered a short duration $S S S$ of $S M L=-3872 \mathrm{nT}$. A GIC of $49 \mathrm{~A}$ occurred at the time of the shock/SSS onset.

A second short duration $S M L=-2724 \mathrm{nT}$ SSS occurred in the storm main phase. It is associated with a double GIC event with peaks of $33 \mathrm{~A}$ and $27 \mathrm{~A}$. There are two clusters of GICs with $>10 \mathrm{~A}$ intensities in the storm recovery phase. They are associated with substorm intervals of peak SML intensities of $-1821 \mathrm{nT}$ and $-797 \mathrm{nT}$, respectively. In the first cluster there is a GIC of $30 \mathrm{~A}$ at 0213 UT on day 304. In the second cluster 


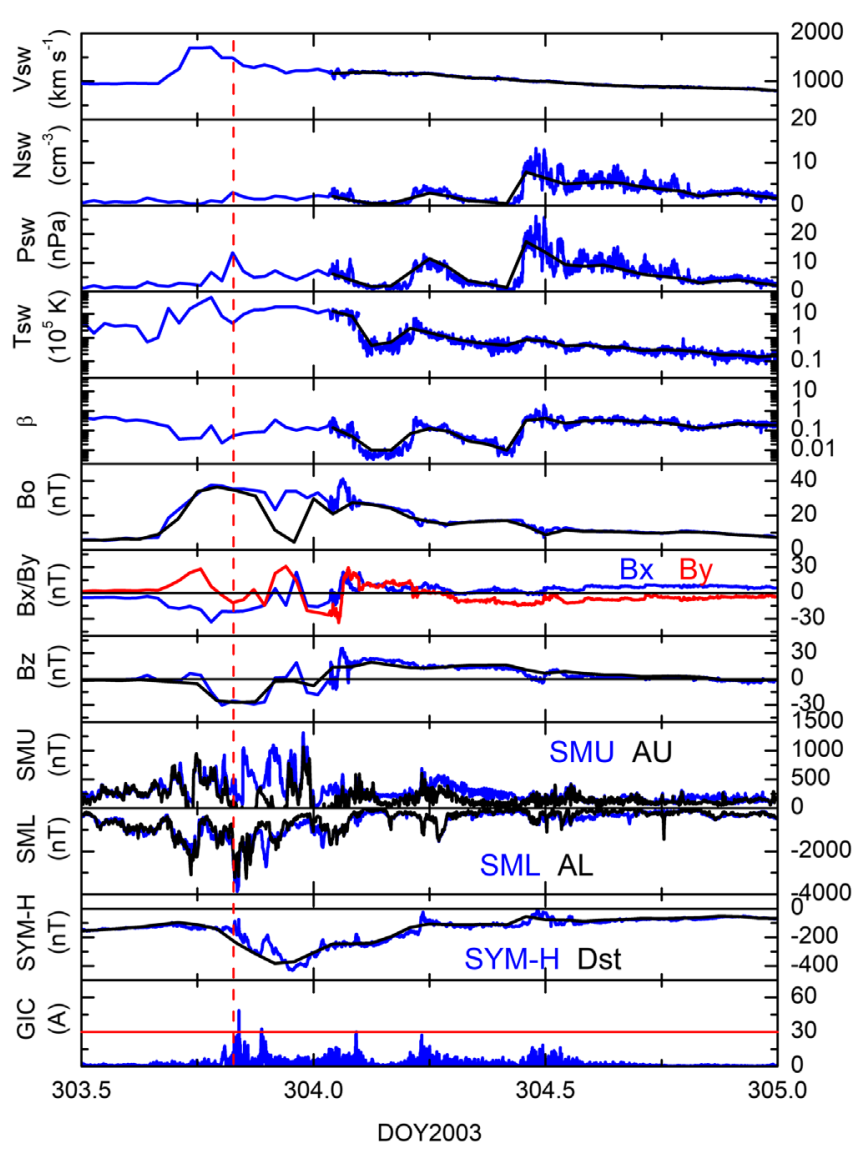

Fig. 3. Same format as in Figure 2, but for the magnetic storm on days 303-304 (30-31 October), 2003. This is the second Halloween magnetic storm. The vertical red dashed line indicates a density spike (PP) incidence.

there is a $27 \mathrm{~A} \mathrm{GIC}$ at $0536 \mathrm{UT}$ on day 304. There is another GIC cluster, well after storm recovery, with 16 A at 1119 $\mathrm{UT}, 19 \mathrm{~A}$ at $1152 \mathrm{UT}, 14 \mathrm{~A}$ at $1227 \mathrm{UT}$ and $16 \mathrm{~A}$ at 1246 UT on day 304.

There were 90 GICs with > 10 A intensities on day 304 . There were 3 GICs with $>30$ A intensities. This second Halloween storm had the second-most GICs in the 21-year study. It should be noted that this second Halloween superstorm had a higher $S Y M-H$ peak intensity than the first superstorm, but had fewer GICs in both intensity categories.

Figure 4 shows a magnetic storm with peak intensity $S Y M-H=-319 \mathrm{nT}$ associated with a complex corotating interaction region (CIR; Smith \& Wolfe, 1976; Tsurutani et al., 1997, 2006). The peak in the storm intensity occurred at $\sim 0011$ UT on day 98. An IMF Bs of intensity $\sim 27$ to $\sim 32 \mathrm{nT}$ was present from the shock (denoted by the vertical black dashed line) to the CIR interface at $\sim 2316$ UT day 97. This Bs caused the main phase of the storm. The strong shock at 1643 UT (1943 LT) created only a sublevel GIC event. Mäntsälä was in the dusk-midnight sector at the time of shock impingement.

The shock also triggered a long-duration intense substorm with peak SML intensity of -2314 nT. A 20 A GIC occurred at the substorm peak.

A short-duration substorm of $S M L=-1844 \mathrm{nT}$ at $2328 \mathrm{UT}$ (day 97) is associated with a GIC intensity of 23 A.

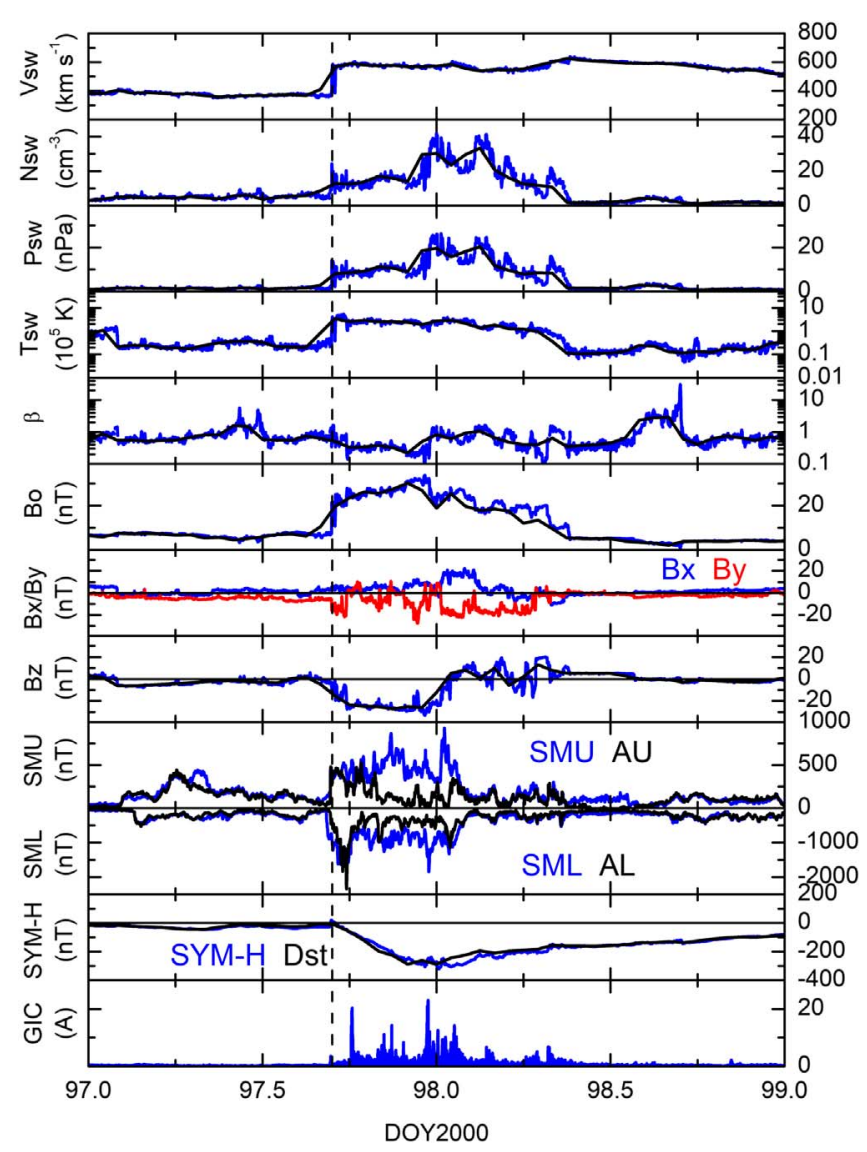

Fig. 4. The format is the same as for Figure 2, but for days 97-98 (6-7 April), 2000.

The substorm spike is not present in the $A L$ index, indicating that either the substorm was located at latitudes below Mäntsälä or that the substorm occurred at a longitude between the widely separated $A L$ index stations. The substorm is coincident in time with an increase in solar wind ram pressure from $\sim 9.5$ to $\sim 25.6 \mathrm{nPa}$. There were $16 \mathrm{GICs}>10$ A intensities that occurred during these two days.

Figure 5 shows an interplanetary sheath Bs causing a SYM$H=-176 \mathrm{nT}$ magnetic storm. The storm peak occurred at $\sim 2104$ UT day 311 . The shock is indicated by a vertical black dashed line at 0958 UT (1248 LT). The shock did not cause a GIC above the study threshold. Mäntsälä was in the noon sector at the time of shock arrival.

A 19 A GIC occurred at 1807 UT on day 311 time-coincident with a solar wind PP with density Nsw $=33 \mathrm{~cm}^{-3}$ (shown by a vertical red dashed line). The upstream solar wind density prior to the PP was $\sim 5 \mathrm{~cm}^{-3}$, so the ram pressure increase across the PP was a factor of $\sim 6.6$ times. This PP may be a coronal loop carried out to $1 \mathrm{AU}$. Such a high level of ram pressure increase is greater than most shock compressions. This GIC event is the largest for this interplanetary interval. Mäntsälä was in the dusk-midnight sector at the time of the GIC. There were only 3 GICs with $>10 \mathrm{~A}$ intensities on these two days.

In Figure 6, the sheath Bs ahead of an ICME caused a magnetic storm of intensity $S Y M-H=-101 \mathrm{nT}$. The MC within the ICME had $\mathrm{Bz} \sim 0 \mathrm{nT}$, so it made the storm only slightly more 


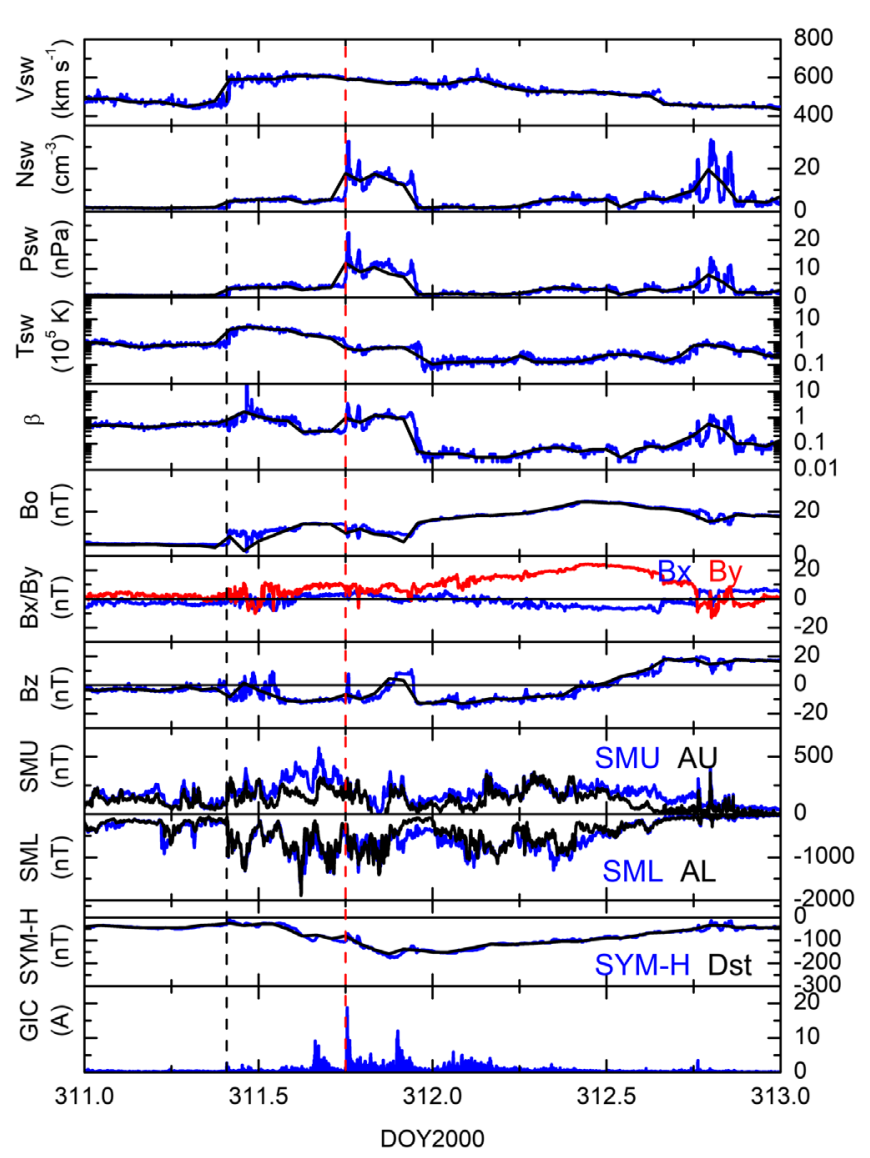

Fig. 5. The same format as in Figure 2 except for days 311-312 (6-7 November), 2000. The vertical red dashed line indicates a PP occurrence.

intense. The storm peak occurred at $\sim 0612$ UT on day 22 . There were 12 GICs with $>10 \mathrm{~A}$ intensities in these two days.

A strong shock at 1702 UT (2012 LT) on 21 January is denoted by a vertical black dashed line. The shock triggered a sharp onset of a SSS with peak SML value of $-4418 \mathrm{nT}$. The shock or the SSS onset caused a GIC of 13 A. Mäntsälä was in the dusk-midnight sector at the time. During the decay phase of the SSS there was a large cluster of GICs reaching a peak intensity of $27 \mathrm{~A}$ at $~ 1910 \mathrm{UT}$. The peak GIC occurred at the same time as a sharp interplanetary density spike (PP) with a peak density of $55 \mathrm{~cm}^{-3}$. The upstream density was $\sim 11 \mathrm{~cm}^{-3}$, thus the ram pressure increases was $\sim 5$ times. This is another example of an abrupt solar wind density increase causing an intense GIC.

There is another GIC of intensity 22 A at 2015 UT on day 21 . There is no obvious interplanetary phenomenon that appears to be the cause. There is no substorm feature at this time.

Following the 22 A GIC is a PP at 2052 UT. This is associated with a heliospheric current sheet (HCS) crossing (Smith et al., 1978). The HCS is identified by sharp reversals in the sign of both IMF $B x$ and $B y$ components. This causes a GIC but below the 10 A threshold of this study.

Figure 7 shows a different type of interplanetary event than the previous examples. This is a "slow" ICME where the peak solar wind speed Vsw is only $\sim 520 \mathrm{~km} \mathrm{~s}^{-1}$ (such slow ICMEs have been noted before: Tsurutani et al., 2004). There is no shock and sheath ahead of the ICME, but there is a "pileup"

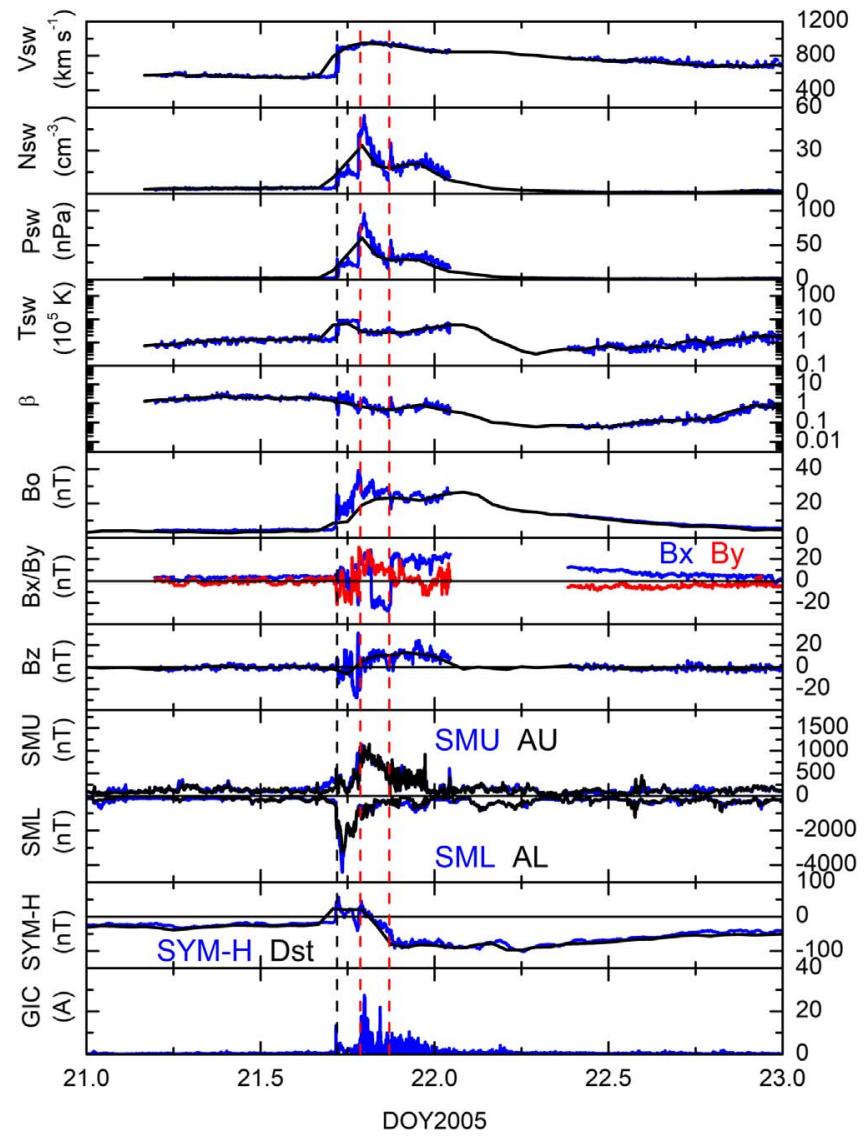

Fig. 6. The format is the same as in Figure 2 but for days 21-22 (2122 January), 2005. The vertical red dashed lines indicate PP events.

region there. The $\mathrm{Bs}$ in the pileup region causes a moderate magnetic storm of $S Y M-H$ peak $=-61 \mathrm{nT}$. The $\mathrm{MC}$ $B z=0 \mathrm{nT}$ causes the lengthening of the storm main and recovery phases. During the storm main phase there are substorms with a peak $S M L$ value of $-848 \mathrm{nT}$. There are no GICs above study threshold in this time interval.

An intense GIC of 20 A occurs at 1924 UT on day 58. The cause of this is a sharp change of the IMF orientation from a $B s$ direction to a $B n$ direction (a $B n$ turning). This occurs at the boundary between the pileup region and the $\mathrm{MC}$ ( $B n$ fields). There is also a solar wind ram pressure decrease at that time associated with the low plasma $\beta$ of the MC. The IMF Bn turning terminates the substorm activity, so there is no substorm associated with this event.

Figure 8 shows another different type of solar wind event, that of an interval of low solar wind speed Vsw with a peak value of only $\sim 420 \mathrm{~km} \mathrm{~s}^{-1}$. There is no shock/sheath, ICME (MC or filament), CIR or HSS involved in this event. Embedded within the low-speed solar wind is a long period $B z$ wave where the IMF Bs reaches $\sim 23 \mathrm{nT}$. This southward IMF leads to a SMY-H $=-153 \mathrm{nT}$ magnetic storm. The storm peak occurs at $\sim 1253$ UT on day 274 . There were 13 GICs with $>10$ A intensities in these two days.

Throughout the relatively long main phase of the magnetic storm and in the beginning of the recovery phase, there is high and continuous substorm activity, noted by the SML indices. A double GIC event of $28 \mathrm{~A}$ and 19 A intensities occurs at 


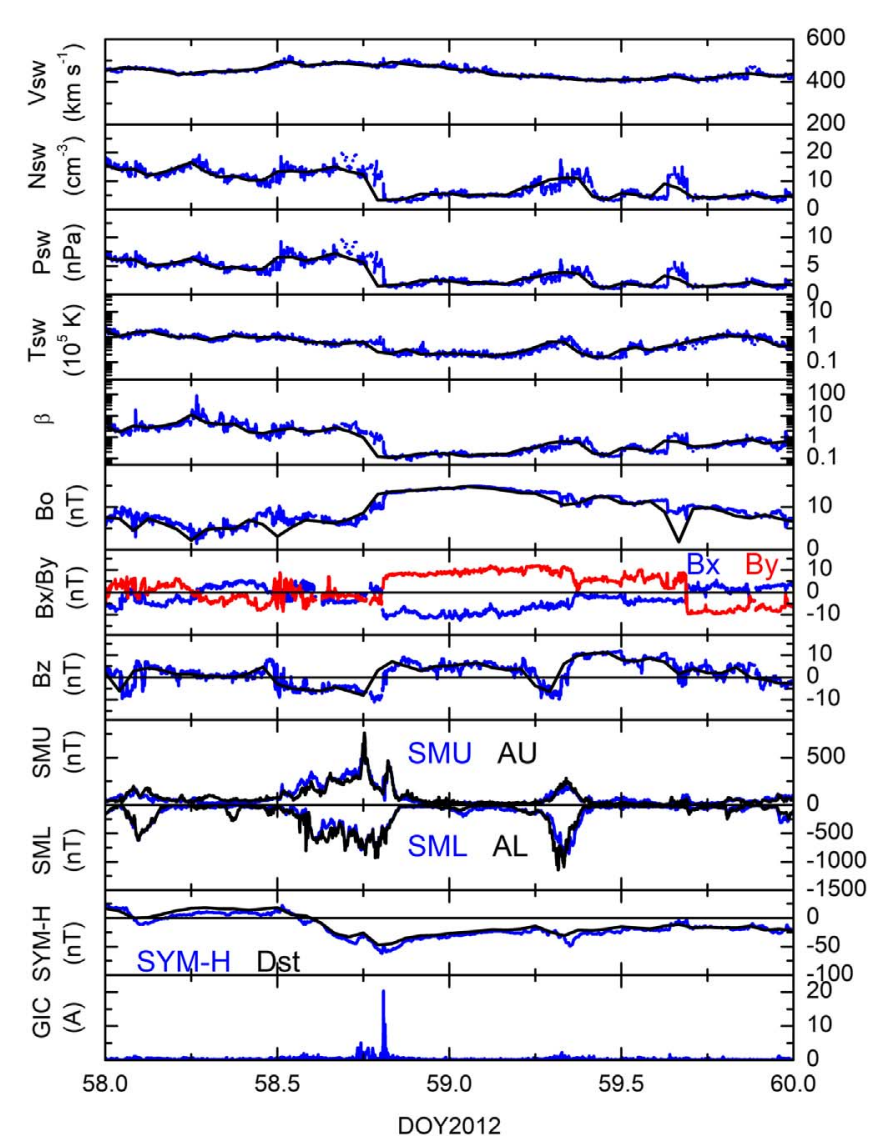

Fig. 7. The format is the same as in Figure 2, but for days 58-59 (27-28 February), 2012.

1629 UT and 1647 UT, respectively. This double GIC is associated with a short duration substorm spike of $S M L=$ $-1906 \mathrm{nT}$. This occurred in the beginning of the storm recovery phase. There is no obvious interplanetary cause of this substorm. There is no particular IMF Bs or Bn turnings or Nsw features present at this time.

\subsection{Analyses using all GIC > 10 A events}

The Appendix shows all of the GIC > 10 A data in 48 plots (Figs. A1 through A48) and summaries. We use this full set of data to obtain some pertinent statistical information.

Almost all of the 48 event intervals were associated with geomagnetic storm intervals. About $21 \%$ were associated with superstorms (SMY-H $\leq-250 \mathrm{nT}$ ), 60\% with intense storms $(-250 \mathrm{nT}<S Y M-H \leq-100 \mathrm{nT}), 17 \%$ with moderate storms $(-100 \mathrm{nT}<S Y M-H \leq-50 \mathrm{nT})$, and $2 \%$ with non-storms $(S Y M-H>-50 \mathrm{nT})$. Although the GIC $>10$ A events were generally associated with storm intervals, they were not necessarily associated with magnetic storm main phases. Examples to the contrary were shown in Figures 2 through 8. There are many such examples in the Appendix.

GICs $>10$ A were found to be associated with shocks, solar wind PPs, and IMF Bn and Bs turnings/intensifications. Many of the intense GIC events were associated with two or more interplanetary features.

To better understand the subset of interplanetary shockrelated GICs $>10 \mathrm{~A}$, the intensity and local times of all events

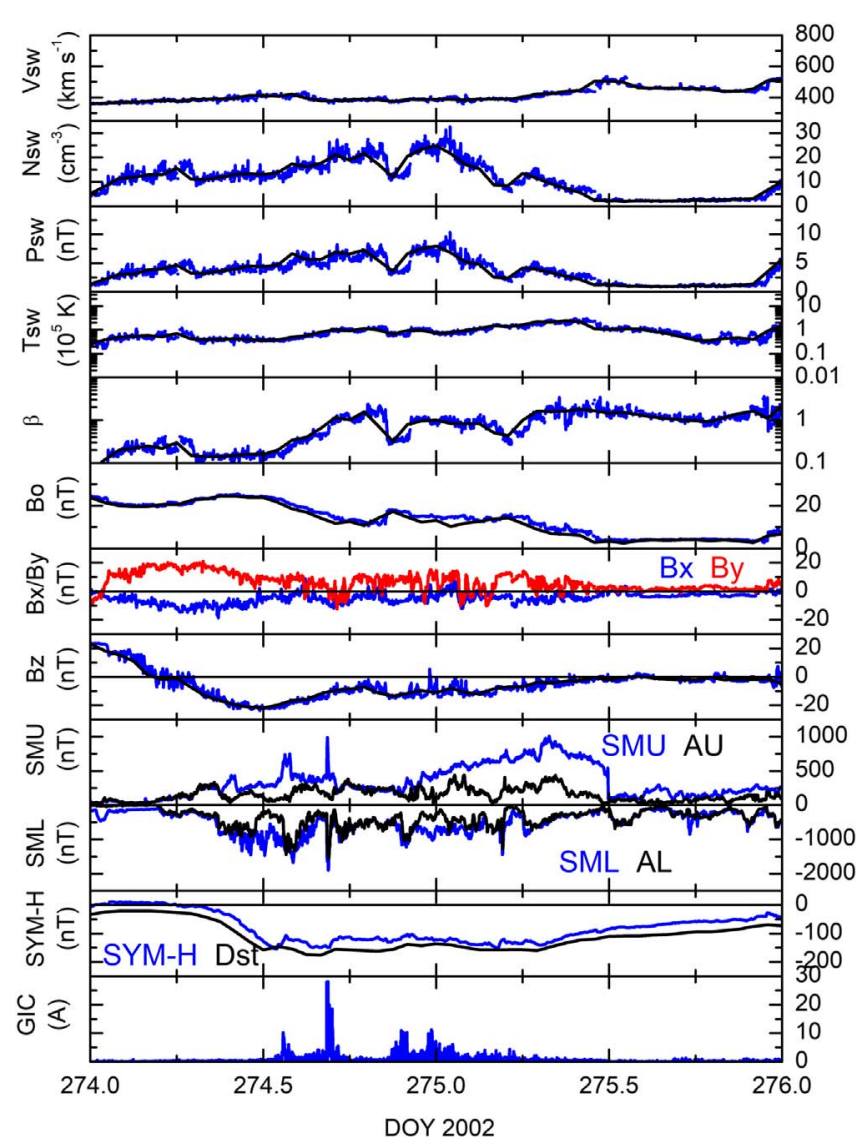

Fig. 8. The format is the same as in Figure 2, but for days 274-275 (1-2 October), 2002.

are indicated in Figure 9. Local noon is at the top of the figure and local midnight at the bottom. The GIC intensity scale is given on the left. It should be noted that all shocks caused a measurable GIC at Mäntsälä (see Appendix and Figs. 2, 4, 5 and 6 for details), but typically below the $10 \mathrm{~A}$ level (there were over 300 shocks that occurred during the study interval). Events with below threshold intensities are not indicated in the above figure.

Figure 9 shows that there were only 11 shock events (out of over 300 ) that were time co-incident with GIC events above the $10 \mathrm{~A}$ level at Mäntsälä. Thus, in general, shocks are not geoeffective at high levels of GIC intensities. This is currently being studied to better understand this feature.

It is noted from Figure 9 that shock-related GICs with intensities $>10 \mathrm{~A}$ can be detected at all local times. If only the most intense events are considered, there is a preference for morning and afternoon hours. However, it is noted that the largest event occurred near the dawn sector.

\subsection{Analyses using all GIC > 30 A events}

It is useful to identify the interplanetary and solar wind causes of the most intense GIC events, those with intensities of $>30 \mathrm{~A}$. This value of $>30 \mathrm{~A}$ is an arbitrarily chosen one.

Figure 10 and Table 1 give the detailed information of all Mäntsälä GICs with $>30 \mathrm{~A}$ intensities. There are only $21>30$ A GIC events in the 21-year study period. Thus, these 


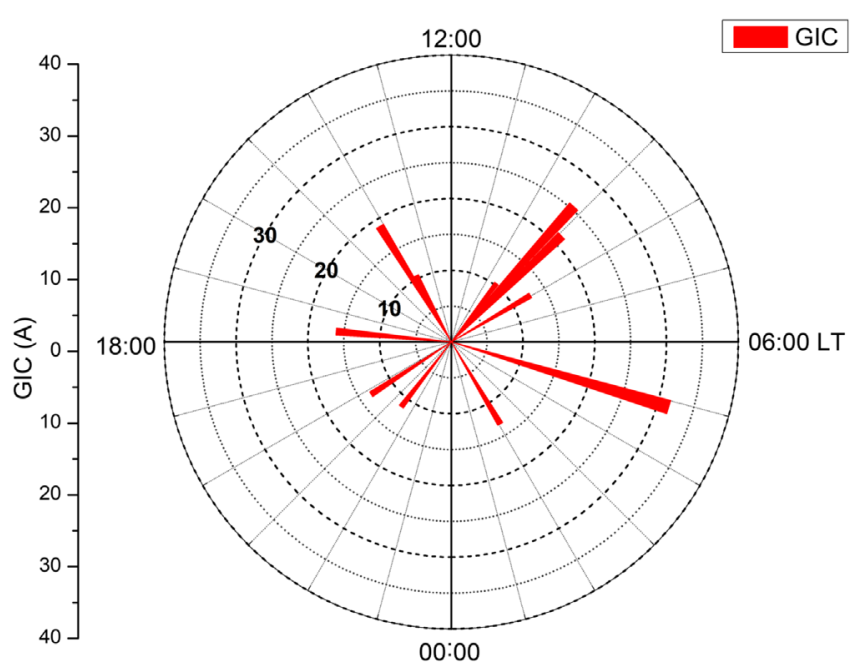

Fig. 9. Interplanetary shock-related Mäntsälä GIC >10 A events. The GIC intensity is indicated by the length of the red radius and the local time by the azimuth angle.

events are quite rare. One of these $>30$ A events was related to a shock and six were related to ram pressure increases caused by PPs. Figure 10 gives the information in a graphic format and Table 1 in a tabular form. The plot format in Figure 10 is the same as for Figure 9 but here all > 30 A GICs are shown independent of whether they were shock-related or not.

The local time distribution of the GICs in Figure 10 can be viewed as having two parts. There is a broad nightside distribution centered roughly at local midnight but extending from $\sim 2000$ LT to $~ 0500$ LT. One might have the suspicion that these events may be substorm-related GICs. There is also a narrow region located in the morning-noon sector, 1000 LT. These GIC events might be suspected to be PP-related events.

It should be noted that 4 of the events (with peaks $33 \mathrm{~A}$, $57 \mathrm{~A}, 51 \mathrm{~A}, 52 \mathrm{~A})$ at $\sim 1000 \mathrm{LT}$ GIC events are those from 29 to 30 October 2003, the first Halloween storm (Table 1). These events were associated with a SSS (see Discussion of Fig. 2). How does a substorm cause dayside GIC events?

Another one of the GIC events (32 A) at $~ 1000$ LT is associated with a large solar wind PP on day 328, 2001 (see Table 1 and Fig. 3 discussion). So, we are seeing GICs (> $30 \mathrm{~A})$ on the dayside associated with both substorms and PPs. It is clear that solar wind shocks and PPs could cause both dayside and nightside GICs. However, substorms causing dayside GICs are interesting and new and needs to be investigated further.

Table 1 shows the details of the $21>30$ A GIC events. The columns are, starting on the far left: the event interval (day/year), the LT, the GIC intensity, the peak magnetic storm $S Y M-H$ intensity, the related substorm peak $S M L$ intensity, and the peak solar wind density Nsw. The density value is only listed if an interplanetary PP was involved in the GIC event, thus the many blank boxes. The sixth column from the left indicates if an interplanetary PP or shock was related to an immediate GIC (a "Yes" response). If the PP or shock triggered a substorm and the substorm intensified with time and was later responsible for the GIC, a "No" is indicated. The next two columns indicate if the GIC is associated with an interplanetary sheath event or an ICME MC event.

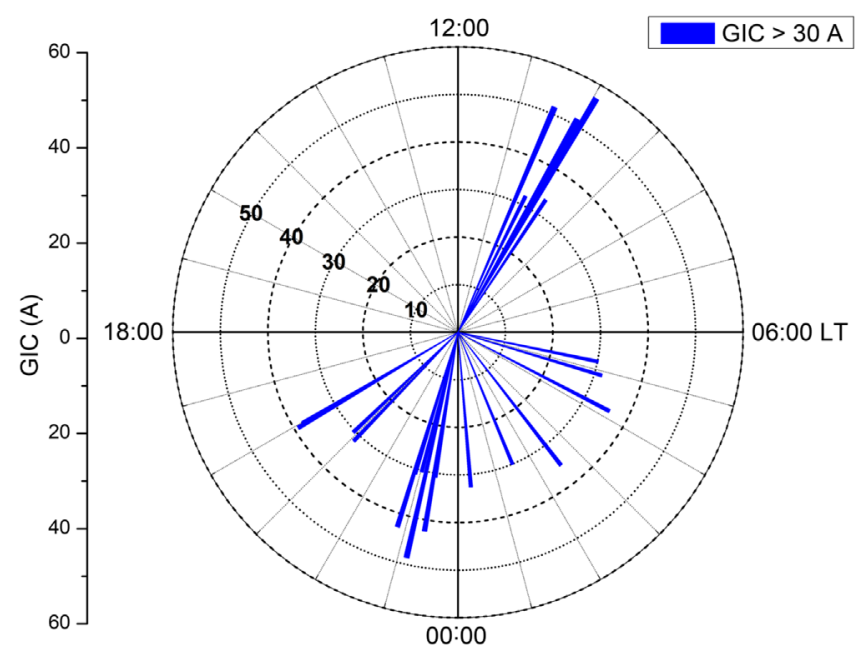

Fig. 10. Local time distribution and intensity of the $>30$ A GIC events at Mäntsälä. The format is the same as in Figure 9.

There are several pertinent features that can be noted from the table. All of the events occurred between years from 2000 to 2004 and from 2012 to 2017 . This corresponds to the solar cycle maximum to the declining phase for the first interval, and from the rising phase to the declining phase for the second interval (see the top panel of Fig. 1 for reference to solar cycle phases). About half of the GIC > $30 \mathrm{~A}$ events are related to sheaths upstream of ICMEs and half to MCs within ICMEs. There were no CIRs involved with these 21 intense GIC events.

Of the $21 \mathrm{GIC}>30$ A events, $16(\sim 76 \%)$ were related to superstorms $(S Y M-H \leq-250 \mathrm{nT})$. Sixteen $(76 \%)$ of the GICs > 30 A were associated with either SSSs $(S M L<$ $-2500 \mathrm{nT})$ or with intense $(-2500 \mathrm{nT}<S M L<-2000 \mathrm{nT})$ substorms. There is one event associated with a shock/superstorm event $(5 \%)$. The other 5 were associated with lesser intensity substorms. From all of the above, it is clear that GICs $>30 \mathrm{~A}$ events are associated with extremely intense magnetospheric and ionospheric geomagnetic activity.

\section{Discussion and conclusions}

\subsection{Superstorms}

Sixteen of the 21 Mäntsälä GIC > 30 A events were associated with superstorms (SYM-H $\leq-250 \mathrm{nT})$. This does not mean that superstorms are causing the GICs, but that the enormous solar wind energy being pumped into the magnetosphere is leading to conditions conducive for the occurrence of particularly strong substorms. The prime examples for this were the first two Halloween storms on 29-30 and 30-31 October 2003 (see also Viljanen et al., 2010 using a different data interval). Between the two superstorms, 9 GICs with intensities $>30 \mathrm{~A}$ and $258 \mathrm{GICs}$ with intensities $>10 \mathrm{~A}$ occurred. These are good fractions ( $43 \%$ and $\sim 43 \%$, respectively) of all of the events detected in the 21 years of study. It should be mentioned that these were not the largest magnetic storms. There were more intense storms with far fewer and considerably lower intensity GIC events. It is noted that the EUV solar flare (28 October 2003) related to the 29-30 October 2003 
Table 1. Details of the GIC > 30 A events under study.

\begin{tabular}{|c|c|c|c|c|c|c|c|c|}
\hline Day/year & $\mathrm{LT}$ & $\begin{array}{c}\text { GIC } \\
\text { peak (A) }\end{array}$ & $\begin{array}{c}S Y M-H \\
\text { peak }(\mathrm{nT})\end{array}$ & $\begin{array}{c}S M L \\
\text { peak }(\mathrm{nT})\end{array}$ & $\operatorname{Nsw}\left(\mathrm{cm}^{-3}\right)$ & Shock/PP & Sheath & $\mathrm{MC}$ \\
\hline $197 / 2000$ & 2302 & 30 & -347 & -3077 & 20.6 & PP & No & Yes \\
\hline $328 / 2001$ & 1014 & 32 & -233 & -3839 & 65.0 & No & No & No \\
\hline $302 / 2003$ & 0946 & 33 & -390 & -3548 & & No & Yes & No \\
\hline $302 / 2003$ & 0957 & 57 & -390 & -3548 & & No & Yes & No \\
\hline $302 / 2003$ & 1003 & 51 & -390 & -3548 & & No & Yes & No \\
\hline $303 / 2003$ & 0410 & 36 & -390 & -2340 & & No & No & Yes \\
\hline $303 / 2003$ & 2309 & 49 & -432 & -3872 & 3.0 & PP & Yes & No \\
\hline $304 / 2003$ & 0019 & 33 & -432 & -2724 & 3.0 & $\mathrm{PP}$ & Yes & No \\
\hline $304 / 2003$ & 0513 & 30 & -432 & -807 & 3.0 & PP & No & Yes \\
\hline $312 / 2004$ & 0231 & 35 & -392 & -2071 & & No & No & Yes \\
\hline $314 / 2004$ & 2251 & 43 & -282 & -2324 & & No & No & Yes \\
\hline $075 / 2012$ & 2003 & 39 & -79 & -1753 & 12.0 & PP & Yes & No \\
\hline $076 / 2013$ & 2105 & 32 & -131 & -990 & & No & Yes & No \\
\hline $251 / 2017$ & 2055 & 30 & -115 & -1223 & & No & No & Yes \\
\hline
\end{tabular}

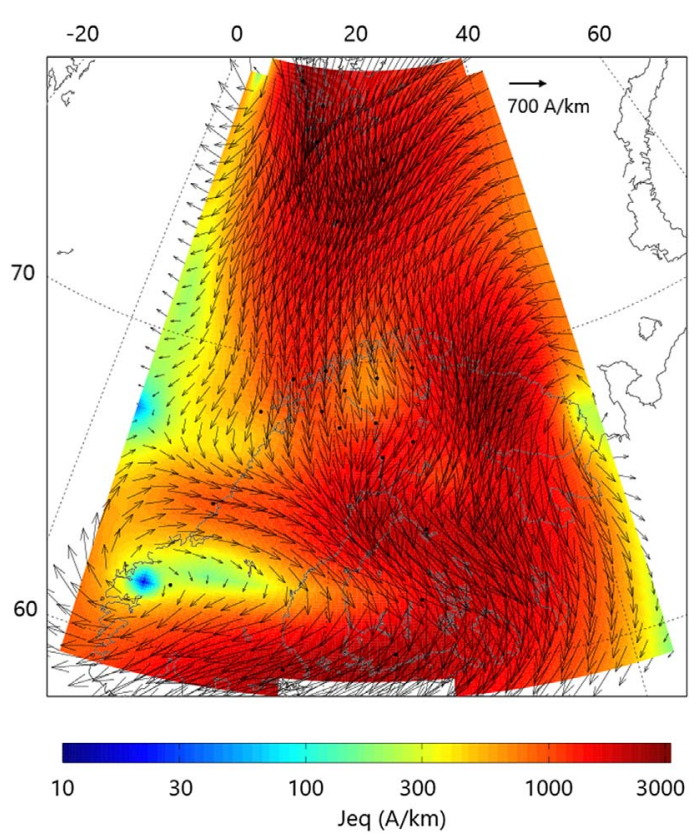

Fig. 11. Ionospheric equivalent currents between $59^{\circ} \mathrm{N}$ and $79^{\circ} \mathrm{N}$ latitudes and $3^{\circ} \mathrm{E}$ to $42^{\circ} \mathrm{E}$ longitudes obtained from the International Monitor for Auroral Geomagnetic Effects (IMAGE) of Space and Earth Observation Centre, Finnish Meteorological Institute (https:// space.fmi.fi/) at 0657 UT on 29 October 2003 (the time of GIC peak of $\sim 57 \mathrm{~A}$ ). The color scale at the bottom shows the magnitude of the eastward currents. The ionospheric equivalent currents, flowing at $\sim 100 \mathrm{~km}$ altitude, are calculated from 41 ground magnetometers (shown by black filled circles) from IMAGE.

Halloween storm was the most intense on record. This caused the greatest dayside flare-related ionospheric Total Electron Content (TEC) increase $(\sim 30 \%)$ on record (Tsurutani et al.,
2005). Mannucci et al. (2005) indicated that the 30-31 October 2003 Halloween superstorm had the largest dayside ionospheric "superfountain" uplift on record. Whether these ionospheric effects caused these two storms to have far more intense GIC events or not is unclear at this time.

Pulkkinen et al. (2005) reported high-voltage power transmission system failures in southern Sweden during the 30-31 October 2003 Halloween superstorm. The interplanetary cause of one GIC reported by Pulkkinen et al. (2005) was identified in this paper. It was a modest solar wind pressure pulse (PP) that created a large $\mathrm{SI}^{+}$in $S Y M-H(+61 \mathrm{nT})$, a $S M L=-3872 \mathrm{nT} S S S$ onset and a 49 A GIC at Mäntsälä, Finland (at 2249 LT). This GIC event was reported earlier relative to Figure 3 and is shown in Figure 10. This was the largest GIC event that occurred during the 30-31 October 2003 superstorm.

\subsection{Substorms, intense substorms and SSSs}

When one narrows the GICs to the most intense $>30 \mathrm{~A}$ events (Table 1), it is apparent that substorms play a major role. It can be noted that 16 of the 21 GIC $>30$ A events are associated with intense substorms with $S M L<-2000 \mathrm{nT}$ intensities. Nine of the events were SSSs $(S M L<-2500 \mathrm{nT})$. Substorms with such large intensities are relatively rare. The largest GIC was 57 A and was associated with a SSS (Fig. 10).

An event not covered in this study interval was the 1989 Hydro Quebec GIC-induced power outage. The magnetic storm was of superstorm intensity (Dst $=-589 \mathrm{nT})$, even larger than any of the storm events covered in this study. Boteler (2019) recently concluded that a shock-triggered SSS caused the GIC which led to the collapse of the electric power grid. Earlier it was noted that magnetic storms were caused by intense magnetic reconnection between interplanetary Bs and the Earth's magnetopause magnetic fields. In this study we have found that shock triggering of intense substorms may initiate storms as well. Nightside injection of energetic plasma deep into the 
magnetosphere will contribute to ring current enhancement and the storm. Possible examples are found in Figures 2, 4, A3, A4, A7 and A26.

\subsection{Interplanetary PPs}

From Table 1, it is noted that 6 out of the 21 Mäntsälä > 30 A GICs were due to PPs. PPs being time-coincident with Mäntsälä GICs is a new discovery. PPs are the second most geoeffective interplanetary cause (after substorms) for $>30$ A GICs. Why were these PPs more geoeffective than shocks? The plasma density increase across a shock is a maximum of $\sim 4$ (Kennel et al., 1985), but statistically only a factor of 1-3 (Tsurutani \& Lin, 1985). Several of the geoeffective PPs were noted to have considerably higher density increases than $\sim 4$.

What are these interplanetary PP structures? It is possible that they are the high-density loops seen in coronagraph images when CMEs are close to the Sun. At 1 AU the coronal loops should appear between the upstream shock and the MC portion of the ICMEs. Events of this type are noted in Figures 5, A9, A18 and A32. Ion charge state analyses will be useful to verify this.

Could some of the PPs be ICME filaments? From the Lepri \& Zurbuchen (2010) filament event interval list, only 2 of the 9 filament events coincided with our intervals of GICs with intensities > 10 A: on day 198, 2000 (Fig. A6) and on day 295, 2001 (Fig. A16). These filaments are shaded in the corresponding figures. Neither of the two filaments were associated with specific GICs above the study threshold.

We have identified other "possible" filaments which were geoeffective in producing Mäntsälä >10 A GICs. Because the events are of short durations, they could have easily been missed in the Lepri \& Zurbuchen (2010) study. These PP events occurred on the sunward side of the MCs. In the text, we have used the modifier "possible" because we have not examined ion charge states data yet. And to further complicate matters, experts indicate that some filaments may not contain definitive ionic features. So, it is possible that we will not be able to obtain a final answer, even with further study.

Since PP impingements onto the magnetosphere is the same physical mechanism (an enhanced ram pressure pulse) as interplanetary shocks (the downstream enhanced densities sunward of forward shocks), it can be expected that PPs will cause GICs in the same locations as shocks have been noted to do: auroral latitudes, midlatitudes and at the equator. Future researchers should be aware of this new development.

\subsection{Shocks}

Shocks played a large role in the intense GIC events at Mäntsälä. There were 11 shock-related GICs > 10 A. However only one of these had an intensity $>30 \mathrm{~A}$ and thus shocks are apparently the third most important phenomena (after substorms and PP events) in this highest GIC intensity category.

There were over $\sim 300$ shocks identified in the study interval. It can be therefore concluded that shocks in general are not typically geoeffective at the Mäntsälä $>10 \mathrm{~A}$ level or $>30 \mathrm{~A}$ level. Of course, this is not to say that shock-induced GIC intensities could not be greater than found in this survey. Tsurutani \& Lakhina (2014) have shown that considerable stronger shocks at the Earth are theoretically possible.

\section{Final comments}

In the literature, it has been assumed that dayside GICs associated with shock impingements onto the magnetosphere are due to a compressional wave propagating through the magnetosphere to the ionosphere causing $\mathrm{d} B / \mathrm{d} t$ current events there (Araki et al., 1993; Tsurutani \& Lakhina, 2014). However, there are other paths that the signals may take. It is known that shocks cause betatron acceleration of pre-existing dayside outer zone magnetospheric $\sim 10$ to $100 \mathrm{keV}$ electrons and protons, accelerate the particles primarily in their perpendicular energies, leading to plasma instabilities (Kennel \& Petschek, 1966; Tsurutani \& Lakhina, 1997), plasma wave generation, and dayside auroral and subauroral and conductivity enhancements (Zhou \& Tsurutani, 1999; Tsurutani et al., 2001a). If dayside auroral oval electric fields exist (Maezawa, 1976), a sudden ionospheric conductivity enhancement will cause dayside GICs much in the same way that the auroral electrojet on the nightside causes GICs. Interplanetary shock impingements also trigger nightside substorms (Heppner, 1955; Zhou \& Tsurutani, 2001; Tsurutani \& Zhou, 2003; Hajra \& Tsurutani, 2018). So, at the present time if a shock impinges upon the magnetosphere and a GIC occurs at the same time, we are not certain which particular physical mechanism caused the GIC.

What is it about the auroral electrojet or auroral particle precipitation that causes the $\mathrm{d} B / \mathrm{d} t$ at the surface of the Earth? Is it a rapid spatial motion of the electrojet current overhead? Or is it a sudden increase or decrease of that current (note that sudden current decreases also cause a $\mathrm{d} B / \mathrm{d} t$ )? Or could it be due to sudden magnetic field-aligned precipitation of electrons or protons into the auroral ionosphere? Substorms involve rapid plasmasheet injections, more spatially limited than that during magnetic storms (Tsurutani \& Gonzalez, 2007). This injection can cause Pc5 and Ps6 pulsations. Which of these possibilities are the causes of GICs? At this time, we do not have answers. Possibly all of the above! And perhaps the causes are different for different substorm GIC events. To obtain definitive answers would require both detailed local magnetic field measurements and auroral all sky images to identify the $\mathrm{d} B / \mathrm{d} t$ causes. This is beyond the scope of the present paper, but has been mentioned for readers interested in doing such detailed studies in the future.

In a converse sense we do not know how the nightside auroral ionospheric currents are connected to the dayside ionosphere. It has been shown that during HILDCAA (high-intensity long-duration continuous auroral activity, Tsurutani \& Gonzalez, 1987) not only are the nightside and dayside auroral zones connected but there are auroras over the polar caps as well (Guarnieri, 2006; Guarnieri et al., 2006a, b). Plasma waves in the polar cap boundary layer show a continuous, unbroken oval circulating the magnetic pole (Tsurutani et al., 1998b, 2001b). Thus, does it make sense that a SSS originating on the nightside might expand and cause a GIC at $~ 1000$ LT on the dayside? Or is it possible that the auroras and the electrojet were only present at 1000 LT for the 29-30 October 2003 SSS? Zhou \& Tsurutani (2004) noted dawn and dusk auroras during a high-speed solar wind stream. They suggested a mechanism of the KelvinHelmholz instability instead of magnetic reconnection for the solar wind energy input into the magnetosphere.

Figure 11 shows the equivalent ionospheric currents flowing at $\sim 100 \mathrm{~km}$ altitude in the northern hemisphere at 0657 UT on 
29 October 2003, corresponding to the 57 A GIC at Mäntsälä (0957 LT). Strong currents of $\sim 3000 \mathrm{~A} \mathrm{~km}^{-1}$ can be noted around Mäntsälä $\left(60.6^{\circ} \mathrm{N}, 25.2^{\circ} \mathrm{E}\right.$ geographic) during the GIC peak occurrence.

What is particularly striking about the $\sim 57$ A GIC event (and other > 30 A GICs during the first SSS of the 29 October 2003 superstorm) was that the GIC occurred when Mäntsälä was on the dayside (see discussion with Fig. 10)! This is not our traditional view of the Akasofu (1964) picture of substorms being confined to the midnight sector.

We mention that for a related event, a shock-triggered SSS $(S M L=-4418 \mathrm{nT})$ occurring on 21 January 2005, Hajra \& Tsurutani (2018) showed that there was a general lack of auroral forms in the midnight sector, and intense auroras at dusk and at dawn. Unfortunately, the images did not go beyond dawn (in Fig. 2 of that paper). Figure 11 above clearly shows strong ionospheric currents flowing on the dayside during the 29 October 2003 SSS at 0657 UT that are most probably related to the intense GIC. It is possible that this may be a new type of "substorm".

As previously mentioned, this paper is intended to be only a top-level survey. Much more detailed work is needed to understand the details of the physical causes of GICs. We hope, the reader will be encouraged to carry out such studies.

For pipeline mitigation techniques at auroral latitudes, we quote Campbell (1980) concerning the Alaska pipeline:

"Pipe corrosion occurs at the underground, exposed pipe points of coating holes and scratches where current can enter or leave the pipe. Such corrosion is proportional to the current density through the exposed points and decreases with the period of the current variation. The Alaska oil pipeline has been protected with sacrificial zinc electrodes that ground the pipe at regularly spaced intervals along the underground route to bleed the current away from the holes in the pipe coating."

The Finnish natural gas pipeline mitigation technique is the same, using sacrificial electrodes (Pirjola \& Lehtinen, 1985). For the Maritimes and Northeast pipeline in New England, they have used a slightly different technique because of the high resistivity of the soil in that region (Rix \& Boteler, 2001). They have used "impressed current type cathode protection rather than passive anodes". From reading some of the current literature (see Gummow \& Eng, 2002; Popov \& Lee, 2018; Googan, 2020), it seems that both of these techniques work quite well.

Concerning more equatorward latitude pipelines, during magnetic storms the electrojet comes down to midlatitudes. Fortunately, these events do not occur very often and when they do, they only last from a few hours to a few days (Gonzalez et al., 1994). Pipelines at subauroral latitudes are not nearly as vulnerable to magnetic storms as are electrical power systems.

Some pertinent comments from previous publications should be given to the readership concerning superstorms. It has been argued in Tsurutani et al. (2020) that it would take a very fast CME to cause a magnetic storm of the intensity of the Carrington 1-2 September 1859 storm (Tsurutani et al., 2003). The Carrington storm had an estimated intensity of Dst -1760 nT, the largest in recorded history and $\sim 3$ times larger than the 1989 Hydro Quebec power outage. The transit time for the CME to go from the Sun to Earth was $\sim 17$ h 40 min (Carrington, 1859). Thus Tsurutani et al. (2020) indicated that CME transit times less than $24 \mathrm{~h}$ would be most important for events of this type. We have identified the transit time of the 29-30 October 2003 CME. The elapsed time from observing the halo CME near Sun at 1130 UT on 28 September (https://cdaw.gsfc.nasa.gov/CME_ list/UNIVERSAL/2003_10/univ2003_10.html) to the detection of the fast forward shock at the Earth's bow shock nose at $\sim 0612$ UT on 29 September, is $\sim 19 \mathrm{~h}$. Thus, it seems that CMEs with transit times of $<\sim 24 \mathrm{~h}$ will be particularly geoeffective in causing GICs at subauroral latitudes. However as shown in the statistical results of this papers GICs at Mäntsälä and storm intensities do not have a one-to-one relationship. The general relationship is, however, good enough to use for forwarnings of possible GIC events. At this time we do not have enough knowledge to make more concrete predictions.

As mentioned previously, this paper is intended as a "toplevel" survey. No effort was made to understand the "details" of the physical causes of GICs. To state that a "shock", a "magnetic storm", "a supersubstorm (SSS)" or "a plasma parcel (PP)" caused or was related to a GIC, is not a detailed understanding of the $\mathrm{d} B / \mathrm{d} t$ that caused a particular GIC. More work needs to be done. But this indeed can be exciting for the young, energetic researchers who wish to make progress in this important field.

Acknowledgements. Portions of this effort were performed at the Jet Propulsion Laboratory, California Institute of Technology under contract with NASA. The work of R.H. is funded by the Science and Engineering Research Board (SERB), a statutory body of the Department of Science and Technology (DST), Government of India through the Ramanujan Fellowship. B.T.T. thanks Prof. A. Nishida for helpful scientific discussions. B.T.T. also thanks Prof. S.I. Akasofu for helpful scientific discussions. The Mäntsälä GIC data were collected from the Space and Earth Observation Centre of the Finnish Meteorological Institute (https://space.fmi.fi/gic/index.php). The solar wind and interplanetary magnetic field data were obtained from the OMNI website (https://omniweb.gsfc.nasa. gov/). The solar wind data for the 29-30 October and 30-31 October 2003 "Halloween storms" were obtained from R. Skoug. The geomagnetic $S Y M-H, D s t, A L$ and $A U$ indices were obtained from the World Data Center for Geomagnetism, Kyoto, Japan (http://wdc.kugi.kyoto-u.ac.jp/). The $S M L$ and $S M U$ indices were taken from the SuperMAG network (http://supermag.jhuapl.edu/). Ionospheric equivalent currents were obtained from the International Monitor for Auroral Geomagnetic Effects (IMAGE) of Space and Earth Observation Centre, Finnish Meteorological Institute (https:// space.fmi.fi/). The editor thanks two anonymous reviewers for their assistance in evaluating this paper.

\section{References}

Akasofu SI. 1964. The development of the auroral substorm. Planet Space Sci 12: 273-282. https://doi.org/10.1016/0032-0633(64) 90151-5.

Araki T, Funato K, Iguchi T, Kamei T. 1993. Direct detection of solar wind dynamic pressure effect on ground geomagnetic field. Geophys Res Lett 20: 775-778. https://doi.org/10.1029/93GL00852. 
Barlow WH. 1849. On the spontaneous electrical currents observed in the wires of the electric telegraph. Phil Trans R Soc Lond 139: 61-72. https://doi.org/10.1098/rstl.1849.0006.

Boteler DH. 2019. A $21^{\text {st }}$ century view of the March 1989 magnetic storm. Space Weath 17: 1427-1441. https://doi.org/10.1029/ 2019SW02278.

Burlaga L, Sittler E, Mariani F, Schwenn R. 1981. Magnetic loop behind an interplanetary shock: Voyager, Helios, and IMP 8 observations. J Geophys Res 86: 6673-6684. https://doi.org/ 10.1029/JA086iA08p06673.

Burlaga L, Fitzenreiter R, Lepping R, Ogilvie K, Szabo A, Lazarus A, Steinberg J, Gloeckler G, Howard R, Michels D, Farrugia C, Lin RP, Larson DE. 1998. A magnetic cloud containing prominence material: January 1997. J Geophys Res 103: 277-285. https://doi. org/10.1029/97JA02768.

Campbell WH. 1980. Observation of electric currents in the Alaska oil pipeline resulting from auroral electrojet current sources. Geophys J R Astr Soc 61: 437-449. https://doi.org/10.1111/j.1365246X.1980.tb04325.x.

Carrington RC. 1859. Description of a singular appearance seen in the Sun on September 1, 1859. Mon Not R Astron Soc, XX: 13-15.

Chen PF. 2011. Coronal mass ejections: Models and their observational basis. Living Rev Solar Phys 8: 1-92. http://www.livingreviews.org/lrsp-2011-1.

Daglis IA, Thorne RM, Baumjohann W, Orsini S. 1999. The terrestrial ring current: formation and decay. Rev Geophys 37: 407-438.

Du AM, Tsurutani BT, Sun W. 2008. Anomalous geomagnetic storm of 21-22 January 2005: A storm main phase during northward IMFs. J Geophys Res 113: A10214. https://doi.org/10.1029/ 2008JA013284.

Dungey JW. 1961. Interplanetary magnetic field and the auroral zones. Phys Rev Lett 6: 47-50.

Echer E, Gonzalez WD, Tsurutani BT. 2008a. Interplanetary conditions leading to superintense geomagnetic storms (Dst $\leq-250 \mathrm{nT}$ ) during solar cycle 23. Geophys Res Lett 35: L06S03. https://doi.org/ 10.1029/2007/GL031755.

Echer E, Gonzalez WD, Tsurutani BT, Gonzalez ALC. 2008b. Interplanetary conditions causing intense geomagnetic storms (Dst $\leq-100 \mathrm{nT}$ ) during solar cycle 23 (1996-2006). J Geophys Res 113: A05221. https://doi.org/10.1029/2007JA012744.

Farrugia CJ, Erkaev NV, Biernat HK, Burlaga LF, Lepping RP, Osherovich VA. 1997. Possible plasma depletion layer ahead of an interplanetary ejecta. J Geophys Res 102: 7087-7093. https://doi. org/10.1029/96JA03822.

Gonzalez WD, Tsurutani BT. 1987. Criteria of interplanetary parameters causing intense magnetic storms (Dst $<-100 \mathrm{nT}$ ). Planet Space Sci 35: 1101-1109. https://doi.org/10.1016/00320633(87)90015-8.

Gonzalez WD, Tsurutani BT, Gonzalez ALC, Smith EJ, Tang F, Akasofu SI. 1989. Solar wind-magnetosphere coupling during intense magnetic storms (978-1979). J Geophys Res 94: 88358851. https://doi.org/10.1029/JA094iA07p08835.

Gonzalez WD, Joselyn JA, Kamide Y, Kroehl HW, Rostoker G, Tsurutani BT, Vasyliunas VM. 1994. What is a geomagnetic storm? J Geophys Res 99: 5771-5792. https://doi.org/10.1029/ 93JA02867.

Gonzalez WD, Echer E, Gonzalez ALC, Tsurutani BT. 2007. Interplanetary origin of intense geomagnetic storms (Dst $\leq-100 \mathrm{nT}$ ) during solar cycle 23. Geophys Res Lett 34: L06101. https://doi.org/ 10.1029/2006GL028879.

Googan C. 2020. The cathodic protection potential criteria: Evaluation of the evidence. Mat Corr. https://doi.org/10.1002/maco.202011978.
Guarnieri FL. 2006. The nature of auroras during High-Intensity Long-Duration Continuous AE Activity (HILDCAA) events: 1998 to 2001. In: Recurrent Magnetic Storms: Corotating Solar Wind Streams, vol. 167, Tsurutani BT, et al., (Eds.) Geophys. Mon. Ser., Amer. Geophys., Un. Press, Washington DC. pp. 235-243. https://doi.org/10.1029/167GM19

Guarnieri FL, Tsurutani BT, Echer E, Gonzalez WD. 2006a. Geomagnetic activity and auroras caused by high-speed streams: A review. In: $A d v$ Geosci, Vol. 8, edited by Duldig et al., World Sci Publ. Co. pp. 91-102.

Guarnieri FL, Tsurutani BT, Gonzalez WD, Gonzalez ALC, Grande M, Soraas F, Echer E. 2006b. ICME and CIR storms with particular emphases on HILDCAA events. In: ILWS Workshop 2006, GOA.

Gummow RA, Eng P. 2002. GIC effects on pipeline corrosion and corrosion control systems. J Atmos Sol-Terr Phys 64: 1755-1764.

Hajra R, Tsurutani BT. 2018. Interplanetary shocks inducing magnetospheric supersubstorms (SML $<-2500 \mathrm{nT}$ ): Unusual auroral morphologies and energy flow. Astrophys $J$ 858: 123. https://doi.org/10.3847/1538-4357/aabaed.

Heppner JP. 1955. Note on the occurrence of worldwide SSCs during the onset of negative bays at College, Alaska. J Geophys Res $\mathbf{6 0}$ : 29-32. https://doi.org/10.1029/JZ060i001p00029.

Illing RME, Hundhausen AJ. 1986. Disruption of a coronal streamer by an eruptive prominence and coronal mass ejection. J Geophys Res 91: 10951-10960. https://doi.org/10.1029/JA091iA10p10951.

Kennel CF, Petschek HE. 1966. Limit on stable trapped particle fluxes. J Geophys Res 71: 1-28. https://doi.org/10.1029/JZ071i001p00001.

Kennel CF, Scarf FL, Coroniti FV, Russell CT, Wenzel K-P, Sanderson TR, Van Ness P, Feldman WC, Parks GK, Smith EJ, Tsurutani BT, Mozer FS, Temerin M, Anderson RR, Scudder JD, Scholer M. 1984a. Plasma and energetic particle structure upstream of a quasi-parallel interplanetary shock. $J$ Geophys Res 89: 5419-5435.

Kennel CF, Edmiston JP, Scarf FL, Coroniti FV, Russell CT, Smith EJ, Tsurutani BT, Scudder JD, Feldman WC, Anderson RR, Mozer FS, Temerin M. 1984b. Structure of the November 12, 1978, quasi-parallel interplanetary shock. J Geophys Res 89: 5436-5452. https://doi.org/10.1029/JA089iA07p05436.

Kennel CF, Edmiston JP, Hada T. 1985. A quarter century of collisionless shock research. In: Collisionless Shocks in the Heliosphere: a Tutorial Review, vol. 34, Stone RGTsurutani BT, (Eds.) Amer. Geophys., Un. Press, Washington DC. pp. 1. https://doi.org/10.1029/GM034p0001

Lepri ST, Zurbuchen TH. 2010. Direct observational evidence of filament material within interplanetary coronal mass ejections. Astrophys J Lett 723: L22-L27. https://doi.org/10.1088/20418205/723/1/L22.

Maezawa K. 1976. Magnetospheric convection induced by the positive and negative $\mathrm{z}$ components of the interplanetary magnetic field: Quantitative analysis using polar cap magnetic records. J Geophys Res 81: 2289-2303. https://doi.org/10.1029/JA081i013p02289.

Mannucci AJ, Tsurutani BT, Iijima BA, Komjathy A, Saito A, Gonzalez WD, Guarnieri FL, Kozyra JU, Skoug R. 2005. Dayside global ionospheric response to the major interplanetary events of October 29-30, 2003 "Halloween storms". Geophys Res Lett 32: L12S02. https://doi.org/10.1029/2004GL021467.

Meng C-I, Tsurutani B, Kawasaki K, Akasofu S-I. 1973. Crosscorrelation analysis of the $\mathrm{AE}$ index and the interplanetary magnetic field Bs component. J Geophys Res 78: 617-628.

Meng X, Tsurutani BT, Mannucci AJ. 2019. The solar and interplanetary causes of superstorms (Minimum Dst $\leq-250 \mathrm{nT}$ ) during the space age. J Geophys Res 124: 3926-3948. https://doi. org/10.1029/2018JA026425. 
Pirjola R, Lehtinen M. 1985. Currents produced in the Finnish $400 \mathrm{kV}$ power transmission grid and in the Finnish natural gas pipeline by geomagnetically-induced electric fields. Ann Geophys 3: 485-491.

Popov BN, Lee J-W. 2018. Cathodic protection of pipelines. In: Handbook of Environmental Degradation of Materials, Elsevier. https://doi.org/10.1016/B978-0-323-52472-8.00025-3.

Pulkkinen A, Viljanen A, Pajunpaa K, Pirjola R. 2001. Recordings and occurrence of geomagnetically induced currents in the Finnish natural gas pipeline network. J Appl Geophys 48: 219-231. https://doi.org/10.1016/S0926-9851(01)00108-2.

Pulkkinen A, Lindahl S, Viljanen A, Pirjola R. 2005. Geomagnetic storm of 29-31 October 2003: Geomagnetically induced currents and their relation to problems in the Swedish high-voltage power transmission system. Space Weath 3: S08C03. https://doi.org/ 10.1029/2004SW000123.

Rix BC, Boteler DH. 2001. Telluric current considerations in the design for the Maritimes and Northeast pipeline. In: Paper presented at the Corrosion 2001, Houston, Texas, March 2001.

Smith EJ, Wolfe JH. 1976. Observations of interaction regions and corotating shocks between one and five AU: Pioneers 10 and 11. Geophys Res Lett 3: 137-140.

Smith EJ, Tsurutani BT, Rosenberg RL. 1978. Observations of the interplanetary sector structure up to heliographic latitudes of 16 : Pioneer 11. J Geophys Res 83(A2): 717-724.

Stone RG, Tsurutani BT. 1985. Collisionless shocks in the heliosphere: A tutorial review. Am Geophys Un Mon Ser (AGU), vol 35, Washington DC.

Tsurutani BT, Gonzalez WD. 1987. The causes of high-intensity long-duration continuous AE activity (HILDCAAs): interplanetary Alfvén wave trains. Planet Space Sci 35: 405-412. https://doi.org/ 10.1016/0032-0633(87)90097-3.

Tsurutani BT, Gonzalez WD. 1995. The efficiency of viscous interaction between the solar wind and the magnetosphere during intense northward IMF events. Geophys Res Lett 22: 663-666. https://doi.org/10.1029/95GL00205.

Tsurutani BT, Gonzalez WD. 1997. The interplanetary causes of magnetic storms: A review. In: Magnetic Storms, vol. 98, Tsurutani BT, Gonzalez WD, Amer. Geophys., Un. Press, Washington DC. pp. 77. https://doi.org/10.1029/GM098p0077.

Tsurutani BT, Gonzalez WD. 2007. A new perspective on the relationship between substorms and magnetic storms. In: $A d v$. Geosci., Vol. 8, Sol. Terr., edited by M. Duldig et al., World Sci. Publ. Co., pp. 1-21.

Tsurutani BT, Lakhina GS. 1997. Some basic concepts of waveparticle interactions in collisionless plasmas. Rev Geophys 35: 491-502.

Tsurutani BT, Lakhina GS. 2014. An extreme coronal mass ejection and consequences for the magnetosphere and Earth. Geophys Res Lett 41: 287-292. https://doi.org/10.1002/2013GL058825.

Tsurutani BT, Lin RP. 1985. Acceleration of $>47 \mathrm{keV}$ ions and $>2$ keV electrons by interplanetary shocks at 1 AU. J Geophys Res $\mathbf{9 0 :}$ $1-11$.

Tsurutani BT, Meng C-I. 1972. Interplanetary magnetic-field variations and substorm activity. J Geophys Res 77: 2964-2970.

Tsurutani BT, Stone RG. 1985. Collisionless shocks in the heliosphere: Review of current research. In: Amer Geophys, Vol. 34, Un. Press: Washington, DC.

Tsurutani BT, Zhou XY. 2003. Interplanetary shock triggering of substorms: WIND and POLAR. Adv Space Res 31: 1063-1067. https://doi.org/10.1016/S0273-1177(02)00796-2.

Tsurutani BT, Gonzalez WD, Tang F, Akasofu SI, Smith EJ. 1988. Origin of interplanetary southward magnetic fields responsible for major magnetic storms near solar maximum (1978-1979). J Geophys Res 95: 8519-8531. https://doi.org/10.1029/JA093iA08p08519.

Tsurutani BT, Gonzalez WD, Tang F, Lee YT. 1992. Great magnetic storms. Geophys Res Lett 19: 73-76. https://doi.org/10.1029/ 91GL02783.

Tsurutani BT, Kamide Y, Gonzalez WD, Arballo JK. 1997. Magnetic Storms. Amer. Geophys. Un. Press, Washington DC. pp. 98.

Tsurutani BT, Arballo JK, Lakhina GS, Ho CM, Ajello J, Pickett JS, Gurnett DA, Lepping RP, Peterson WK, Rostoker G, Kamide Y, Kokubun S. 1998a. The January 10, 1997 auroral hot spot, horseshoe aurora and first substorm: A CME loop? Geophys Res Lett 25: 3047-3050. https://doi.org/10.1029/98GL01304.

Tsurutani BT, Lakhina GS, Ho CM, Arballo JK, Galvan C, Boonsiriseth A, Pickett JS, Gurnett DA, Peterson WK, Thorne RM. 1998b. Broadband plasma waves observed in the polar cap boundary layer: POLAR. J Geophys Res 103: 17351-17366. https://doi.org/10.1029/97JA03063.

Tsurutani BT, Zhou XY, Vasyliunas VM, Haerendel G, Arballo JK, Lakhina GS. 2001a. Interplanetary shocks, magnetopause boundary layers and dayside auroras: The importance of a very small magnetospheric region. Surv. Geophys. 22: 101-130, Kluwer Publ., Netherlands. https://doi.org/10.1023/A:1012952414384.

Tsurutani BT, Arballo JK, Galvan C, Zhang LD, Zhou XY. 2001b. Polar cap boundary layer waves: An auroral zone phenomenon. J Geophys Res 106: 19035-19055. https://doi.org/10.1029/2000JA003007.

Tsurutani BT, Gonzalez WD, Lakhina GS, Alex S. 2003. The extreme magnetic storm of 1-2 September 1859. J Geophys Res 108: 1268. https://doi.org/10.1029/2002JA009504.

Tsurutani BT, Gonzalez WD, Guarnieri F, Kamide Y, Zhou X, Arballo JK. 2004. Are high-intensity long-duration continuous AE activity (HILDCAA) events substorm expansion events? J Atmos Sol Terr Phys 66: 167-176. https://doi.org/10.1016/j.jastp.2003.08.015.

Tsurutani BT, Judge DL, Guarnieri FL, Gangopadhyay P, Jones AR, Nuttall J, Zambon GA, Didkovsky L, Mannucci AJ, Iijima B, Meier RR, Immel TJ, Woods TN, Prasad S, Floyd L, Huba J, Solomon SC, Straus P, Viereck R. 2005. The October 282003 extreme EUV solar flare and resultant extreme ionospheric effects: Comparison to other Halloween events and the Bastille Day event. Geophys Res Lett 32: L03S09. https://doi.org/10.1029/2004GL021475.

Tsurutani BT, McPherron RL, Gonzalez WD, Lu G, Sobral JHA, Gopalswamy N. 2006. Corotating solar wind streams and recurrent geomagnetic activity. Am. Geophys., Un. Press, Washington DC. pp. 167.

Tsurutani BT, Lakhina GS, Verkhoglyadova OP, Gonzalez WD, Echer E, Guarnieri FL. 2011. A review of interplanetary discontinuities and their geomagnetic effects. J Atmos Sol Terr Phys 73: 5-19. https://doi.org/10.1016/j.jastp.2010.04.001.

Tsurutani BT, Hajra R, Echer E, Gjerloev JW. 2015. Extremely intense (SML $\leq 22122500 \mathrm{nT}$ ) substorms: isolated events that are externally triggered? Ann Geophys 22: 519-524. https://doi.org/ 10.5194/angeocom-33-519-2015.

Tsurutani BT, Lakhina GS, Hajra R. 2020. The physics of space weather/solar-terrestrial physics (STP): what we know now and what the current and future challenges are. Non Proc Geophys 27: 75-119. https://doi.org/10.5194/npg-27-75-2020.

Viljanen A, Pulkkinen A, Pirjola R, Pajunpaa K, Posio P, Koistinen A. 2006. Recordings of geomagnetically induced currents and a nowcasting service of the Finnish natural gas pipeline system. Space Weath 4: S10004. https://doi.org/10.1029/2006SW000234. Viljanen A, Koistinen A, Pajunpää K, Pirjola R, Posio P, Pulkkinen A. 2010. Recordings of geomagnetically induced currents in the finnish natural gas pipeline - Summary of an 11-year Period. Geophysica 46: 59-67. 
Williams DJ. 1985. Dynamics of the Earth's ring current: theory and observation. Space Sci Rev 42: 375-396. https://doi.org/10.1007/ BF00214994.

Zhang J, Richardson IG, Webb DF, Gopalswamy N, Huttunen E, Kasper JC, Nitta NV, Poomvises W, Thompson BJ, Wu CC, Yahiro S, Zhukov AN. 2007. Solar and interplanetary sources of major geomagnetic storms (Dst $\leq-100 \mathrm{nT}$ ) during 1996-2005. J Geophys Res 112: A10102. https://doi.org/10.1029/2007JA012321.

Zhou X, Tsurutani BT. 1999. Rapid intensification and propagation of the dayside aurora: Large scale interplanetary pressure pulses (fast shocks). Geophys Res Lett 26: 1097-1100. https://doi.org/ 10.1029/1999GL900173.

Zhou X, Tsurutani BT. 2001. Interplanetary shock triggering of nightside geomagnetic activity: Substorms, pseudobreakups, and quiescent events. J Geophys Res 116: 18957-18967. https://doi. org/10.1029/2000JA003028.

Zhou X, Tsurutani BT. 2004. Dawn and dusk auroras caused by gradual solar wind ram pressure events. J Atmos Sol Terr Phys 66: 153-160.

\section{Appendix}

In the narrative below, we discuss the interplanetary and magnetospheric/ionospheric features time-coincident with the Mäntsälä, Finland geomagnetically induced current (GIC) events with intensities $>10 \mathrm{~A}$. We show the interplanetary data so that the readership can note the relationship between GICs $>10$ A and their interplanetary causes. The figures and related text will also describe the Mäntsälä GICs in relation to geomagnetic storm and substorm activity. Many of the GIC events occur before, during, or after magnetic storms, so a broad swath of relevant interplanetary data is shown for each event.

Day 13 (13 January), 1999 (Fig. A1). A CIR caused double dip storm with $S Y M-H$ peak value of $-111 \mathrm{nT}$. The two longduration substorms caused the double dip storm. There is a small sublevel GIC with the forward shock which occurred at 1054 UT (1354 LT). At the time Mäntsälä was in the afternoon sector. A sharp Bs increase caused the second dip of the storm and a $S M L=-1189 \mathrm{nT}$ peak long-duration substorm. A GIC cluster with $10 \mathrm{~A}$ and $13 \mathrm{~A}$ peak intensity events occurred near the end of the substorm with a sharp $S M U$ increase to 469 nT. The storm recovery phase begins after this last substorm.

Day 49 (18 February), 1999 (Fig. A2). Double dip magnetic storm of intensity $S Y M-H=-128 \mathrm{nT}$ (in first dip). The storm is caused by the sheath Bs. The MC causes the second storm dip. A shock occurred at $\sim 0247$ UT (0547 LT) and caused a sublevel GIC. At the time Mäntsälä was in the dawn sector. There was a GIC cluster with peak 12 A event at the beginning of the storm second dip recovery phase. The GIC cluster was associated with a substorm with intensity $S M L=-1604 \mathrm{nT}$.

Days 265-266 (22-23 September), 1999 (Fig. A3). Two shocks, two sheaths, and a MC event. The second sheath Bs caused both a storm of intensity $S Y M-H=-166 \mathrm{nT}$ and a long-duration substorm of $S M L=-1608 \mathrm{nT}$. The shocks at 1223 UT (1523 LT) and 1942 UT (2242 LT) created sublevel GIC events. At the times of the two shocks Mäntsälä was in the afternoon and midnight sectors, respectively. Although the

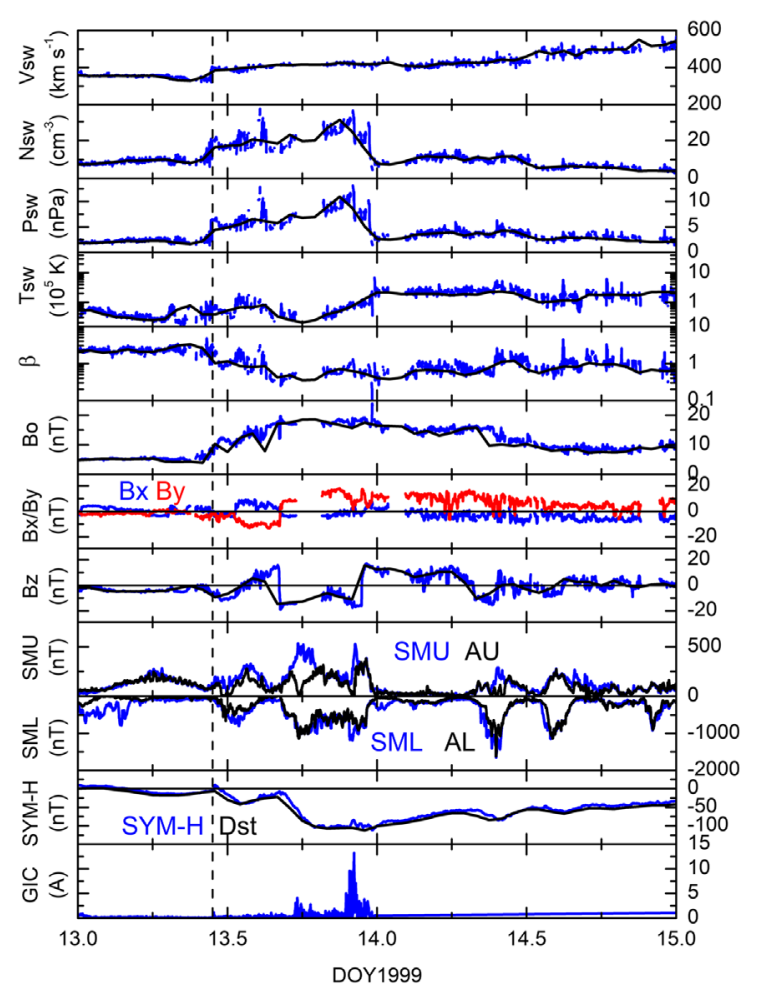

Fig. A1. Events on day 13 (13 January) 1999. From top down are the solar wind velocity (Vsw), density (Nsw), ram pressure (Psw), temperature (Tsw), plasma $\beta$, magnetic field magnitude $(B o)$, the $B x$ and $B y$ components (in the same panel) and $B z$ in GSM coordinates. The bottom four panels are the $S M U$ and $A U$ (in the same panel), $S M L$ and $A L$ (in the same panel), $S Y M-H$ and $D s t$ (in the same panel) and the GIC intensities. In the solar wind/interplanetary and geomagnetic indices panels, blue and black curves show $1 \mathrm{~min}$ and $1 \mathrm{~h}$ resolution data, respectively. The vertical black dashed line indicates the time of the interplanetary shock.

second sheath Bs appears to have caused the magnetic storm, the substorm may have contributed greatly. A cluster of GICs is related to the substorm but there is only one event above threshold, $11 \mathrm{~A}$. The GIC cluster occurs during the storm main phase and the start of the recovery phase (at the end of the substorm).

Days 97-98 (6-7 April), 2000 (Fig. A4). This event was previously discussed in Results section (Fig. 4). A complex CIR associated superstorm with peak intensity $S Y M-H=$ -319 nT at $\sim 0011$ UT day 98. There was a constant Bs of intensity $\sim 30 \mathrm{nT}$ from the shock to the CIR interface at 2316 UT day 97. The shock triggered a long-duration intense substorm with peak SML intensity of $-2314 \mathrm{nT}$. This intense substorm contributed to the storm initial intensification. The strong shock at $\sim 1643$ UT (1943 LT) created a sublevel GIC event. Mäntsälä was in dusk sector at the time. A 20 A GIC occurs at the substorm peak several hours later. A second peak GIC of 23 A was associated with a short duration substorm of $S M L=-1844 \mathrm{nT}$ intensity at $2328 \mathrm{UT}$ day 97 in the storm main phase. This substorm spike was not present in the $A L$ indices. The substorm is coincident in time with a solar wind ram pressure increase to $\sim 26 \mathrm{nPa}$. The high solar wind densities on the 


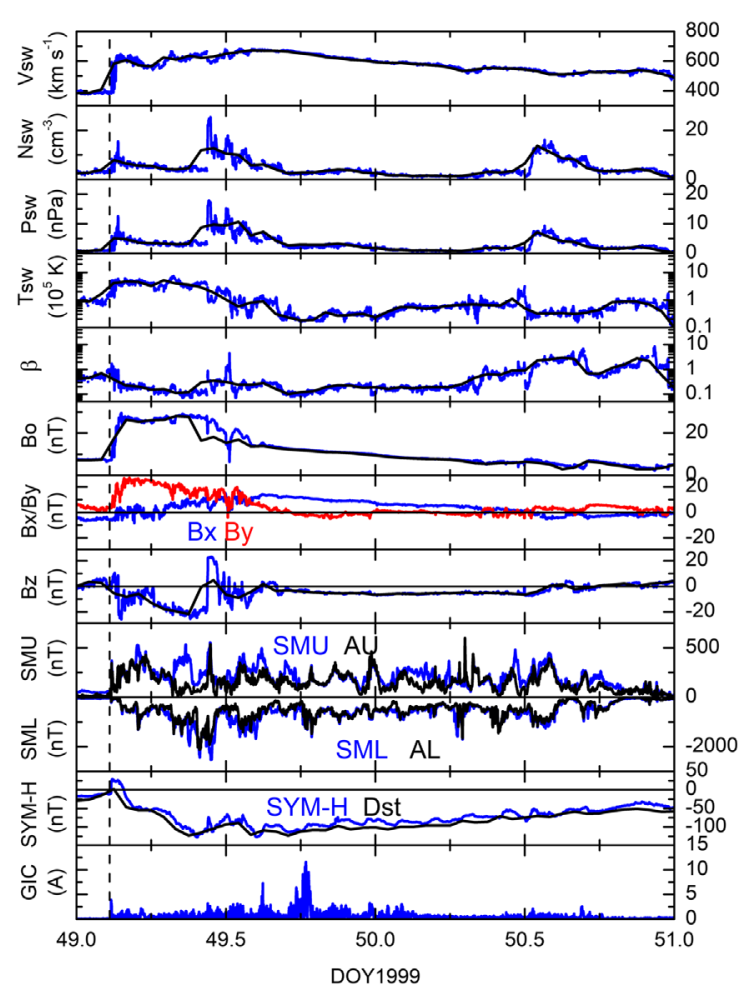

Fig. A2. Events on day 49 (18 February) 1999. Same format as in Figure A1.

solar side of the interface of the CIR is unusual. 16 > 10 A GICs occurred during this interval.

Day 145 (May 24), 2000 (Fig. A5). A complex CIR causes a magnetic storm of peak intensity $S Y M-H=-173 \mathrm{nT}$. The main phase of the storm is caused by a long-duration intense substorm with $S M L=-2121 \mathrm{nT}$ peak intensity. The substorm causes a GIC cluster with two events of $10 \mathrm{~A}$ and $11 \mathrm{~A}$ intensities.

Day 197-198 (July 15-16), 2000 (Fig. A6). A SYM-H= $-347 \mathrm{nT}$ superstorm caused by Bs fields in the upstream sheath and following MC Bs fields. A 16 A GIC is associated with the shock/short-duration $S M L=-2112 \mathrm{nT}$ intense substorm (the shock is noted by a $\mathrm{SI}^{+}$of $84 \mathrm{nT}$ at $\sim 1439$ UT (1739 LT) (the interplanetary data is low resolution for this part of the event). At the time of the shock, Mäntsälä was in the dusk sector. There are many more intense substorms after the shock but no corresponding GICs above the study threshold. The biggest GIC spike of 30 A occurs in beginning of the storm main phase and the decay phase of a short-duration SSS of SML intensity -3077 nT at 1901 UT (2201 LT). Mäntsälä was in the duskmidnight sector at the time. In the storm recovery phase, there is a 17 A GIC associated with a $S M L=-1033 \mathrm{nT}$ substorm. There were $30>10$ A GICs during this event. At 1200 UT day 198, there is an ICME filament indicated by the vertical shading. It is associated with small GICs below the study threshold.

Days 261-262 (17-18 September), 2000 (Fig. A7). A sheath event (without shocks) Bs caused a $S Y M-H=-203$ nT storm.

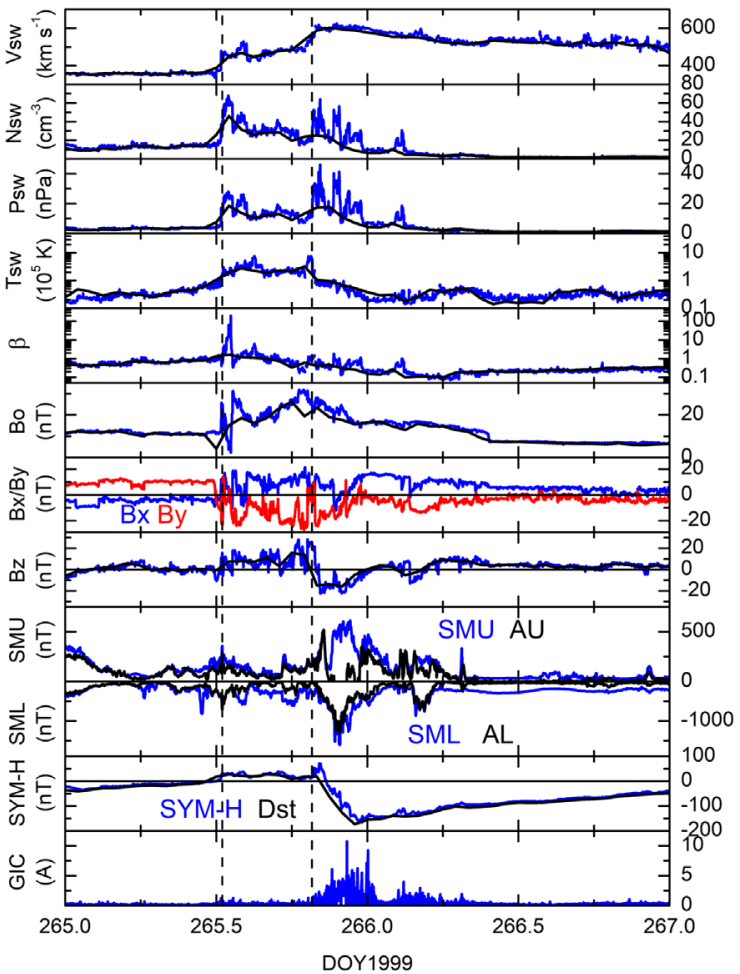

Fig. A3. Events on days 265-266 (22-23 September) 1999. Same format as in Figure A1.

A long-duration intense substorm of $S M L=-2216 \mathrm{nT}$ (or the Bs) caused the storm main phase and also a GIC cluster with 2 peak intensities of $13 \mathrm{~A}$ and $14 \mathrm{~A}$. A GIC of intensity $15 \mathrm{~A}$ occurred at the end of the intense substorm, coincident with an IMF Bs-to-Bn turning. GICs of 13 A at 0342 UT (0642 LT) and $13 \mathrm{~A}$ at $1001 \mathrm{UT}$ (1301 LT) on day 262 were present in the storm recovery phase. Both were not associated with any obviously related substorm or interplanetary feature.

Days 278-279 (4-5 October), 2000 (Fig. A8). Two shocks and two sheaths caused a $S Y M-H=-187$ nT triple dip storm. The weak shock at $\sim 1413$ UT (1713 LT) and pre-existing Bs fields formed the first $-185 \mathrm{nT}$ storm. The shock created a substorm of intensity $S M L=-1435 \mathrm{nT}$ and a GIC of sublevel intensity. The second shock occurred at 0326 UT (0626 LT) on day 279 and caused a sublevel GIC. The second sheath Bs interval creates a $S Y M-H=-187 \mathrm{nT}$ storm and a substorm of $S M L=-1947 \mathrm{nT}$. A second interval of sheath IMF Bs created a $S S S$ of $S M L=-2787 \mathrm{nT}$ and the third (but lesser) dip of the storm. The SSS does not cause a GIC with intensities above threshold. In the storm recovery phase, there is a short-duration substorm with $S M L$ peak intensity of only $-1158 \mathrm{nT}$ that causes a double GIC of $13 \mathrm{~A}$ and $11 \mathrm{~A}$ intensities. This substorm is not apparent in the $A L$ index.

Days 311-312 (6-7 November), 2000 (Fig. A9). This event was previously discussed in Results section (Fig. 5). A constant Bs in a sheath caused a $S Y M-H=-176 \mathrm{nT}$ magnetic storm with peak at $\sim 2104$ UT day 311. The strong shock at $\sim 0948$ UT (1248 LT) did not cause a GIC above study level threshold. Mäntsälä was in the noon sector at the time of the event. 


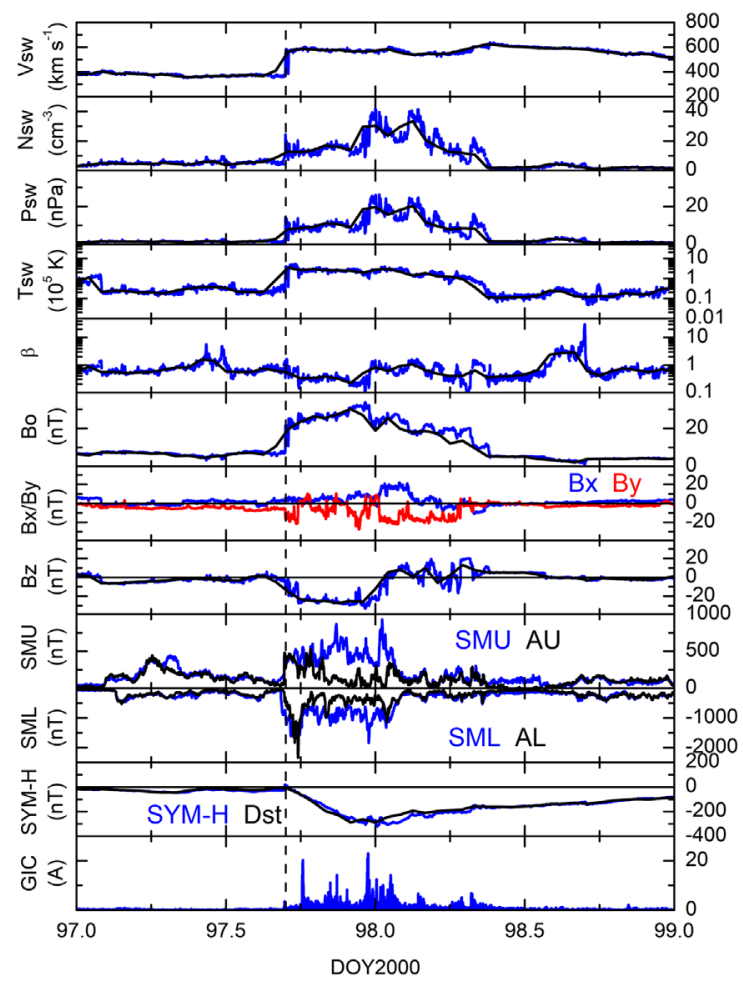

Fig. A4. Events on days 97-98 (6-7 April) 2000. Same format as in Figure A1.

A 19 A GIC occurred in the storm main phase with no apparent strong substorm relationship. An unusual sharp short-duration PP of $33 \mathrm{~cm}^{-3}$ is related to the GIC. This increased the solar wind ram pressure by a factor of 6.6 times. This PP may be a coronal loop that has propagated to $1 \mathrm{AU}$.

Day 315 (10 November), 2000 (Fig. A10). A high-speed $\left(\mathrm{Vsw}=920 \mathrm{~km} \mathrm{~s}^{-1}\right)$ sheath and intermittent Bs fields caused a $S Y M-H=-105 \mathrm{nT}$ magnetic storm. An interplanetary shock at 0629 UT (0929 LT) ahead of an ICME triggers a 10 A GIC spike. Only a moderate intensity substorm was triggered by the shock. Mäntsälä was in the morning sector at the time of the shock. There were no GICs above threshold during the storm main phase. A MC followed the sheath but it had Bn fields and thus leads to the storm recovery phase.

Day 78 (19 March), 2001 (Fig. A11). A double magnetic storm of peak intensity $S Y M-H=-164 \mathrm{nT}$ is caused by a sheath (first dip) and MC (second dip). There is a 16 A GIC in beginning main phase of the storm. The GIC occurred during the decay of a $\mathrm{Nsw}=33 \mathrm{~cm}^{-3}$ solar wind density spike. There is no particularly strong substorm relationship. This GIC event does not have an obvious interplanetary or magnetospheric cause.

Day 90 (31 March), 2001 (Fig. A12). A superstorm of SYM$H=-437 \mathrm{nT}$ was caused by sheath fields ahead of an ICME (whose MC Bs caused a second, less intense storm of $S Y M-H=-269 \mathrm{nT})$. The shock at $\sim 0101 \mathrm{UT}(0401 \mathrm{LT})$ triggered a short-duration substorm of $S M L=-1541 \mathrm{nT}$ and a GIC below threshold. Mäntsälä was in the midnight-dawn sector at the time. Two GICs with 14 A intensities occurred in the

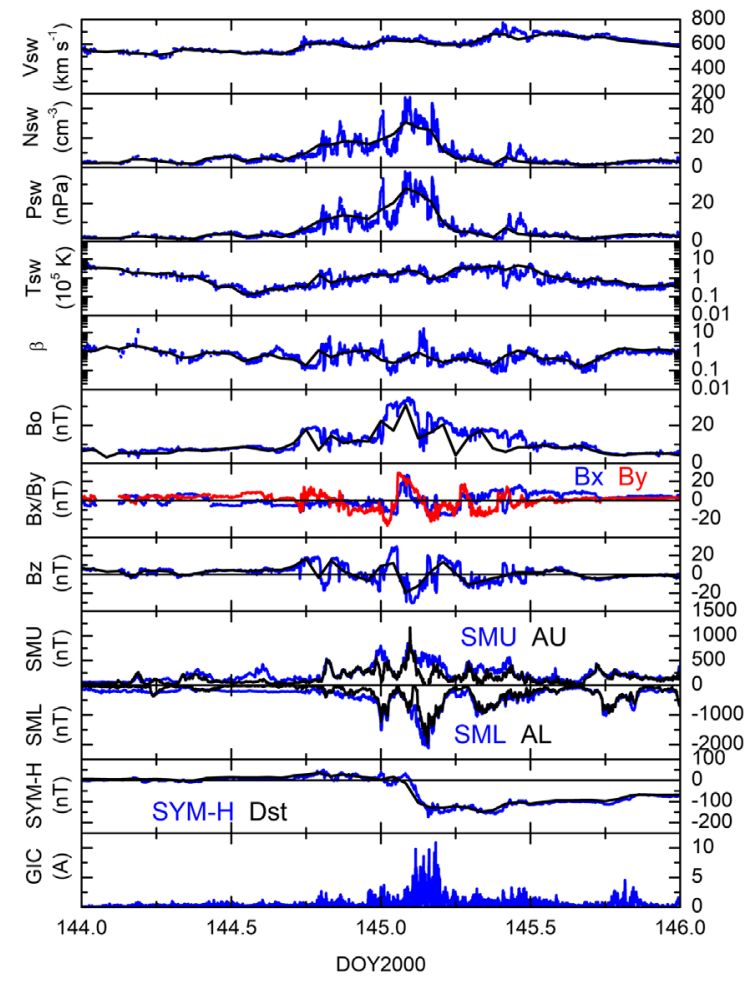

Fig. A5. Events on day 145 (24 May) 2000. Same format as in Figure A1.

initial phase before the storm main phase. These were associated with substorm activity. A 12 A GIC event occurred in the storm main phase. All 3 GIC events were associated with substorm activity. In the second, lesser intensity storm, there are 3 large sharp onset substorms of peak intensities $S M L=-2459$, $-1721 \mathrm{nT}$ and $-1404 \mathrm{nT}$. The first substorm event caused a 13 A GIC. The second and third substorm-related GICs had intensities of $16 \mathrm{~A}$ and $13 \mathrm{~A}$.

Day 101-102 (11-12 April), 2001 (Fig. A13). A shock and sheath with intermittent Bs ahead of a MC caused a superstorm main phase of $S Y M-H=-280 \mathrm{nT}$. The MC begins with intense IMF Bs which may contribute to the peak of the storm main phase. The shock at 1345 UT (1645 LT) triggers a SSS of $S M L=-2923$ nT intensity but there is no GIC above the 10 A threshold. Mäntsälä was in the afternoon-dusk sector at the time. A GIC of 18 A occurs at the beginning of the storm main phase and occurred in a substorm-intense interval. A 22 A GIC occurred later in the storm main phase and is associated with a substorm $S M U=1191 \mathrm{nT}$ peak intensity. This substorm shows up more strongly in $S M U$ rather than SML. A 16 A GIC is noted at the start of the storm recovery phase. This occurred as the substorm activity was subsiding and there was no 1-to-1 relationship with a substorm.

Day 118 (28 April), 2001 (Fig. A14). An ICME event that does not cause a magnetic storm $(S Y M-H=-34 \mathrm{nT})$. The strong shock at $\sim 0502$ UT (0802 LT) caused a SI+ of $75 \mathrm{nT}$. The shock also triggered a sudden onset substorm of $S M L=$ $-498 \mathrm{nT}$ intensity. The $S M U$ intensity was larger, $777 \mathrm{nT}$. A 13 A GIC occurred at the same time as the shock and 


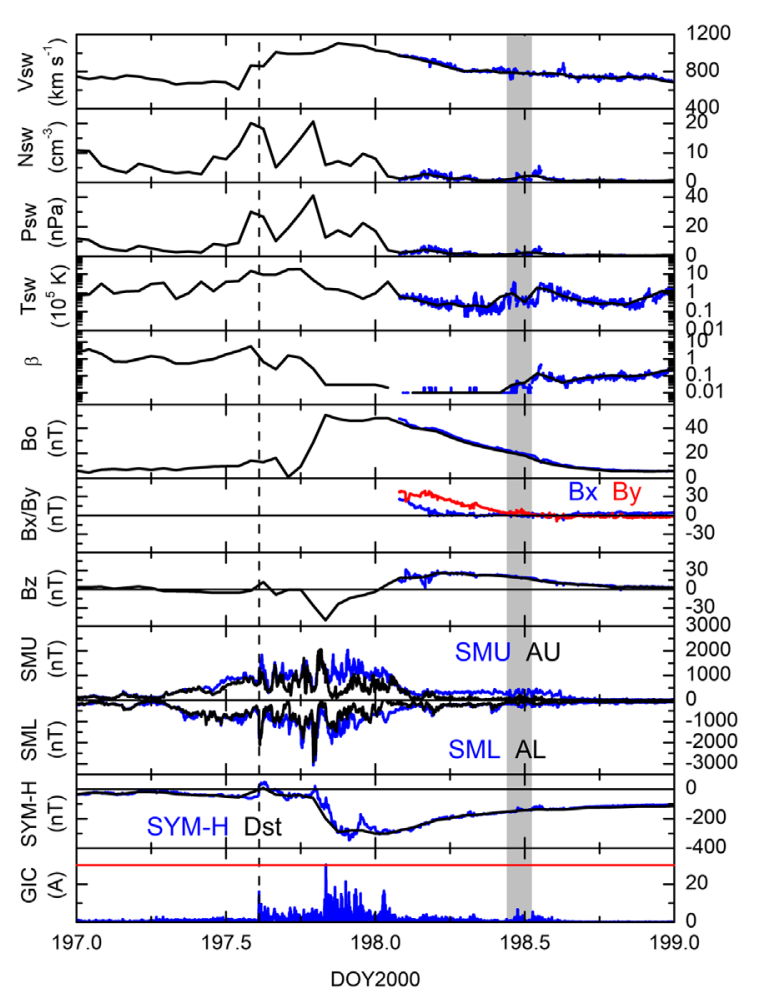

Fig. A6. Events on days 197-198 (15-16 July) 2000. Same format as in Figure A1. The horizontal red line in GIC panel indicates the GIC $=30$ A level. The vertical shading represents a ICME filament identified by Lepri \& Zurbuchen (2010).

substorm sudden onset. At the time of the shock, Mäntsälä was in the morning sector. There was no intense Bs in the sheath or $\mathrm{MC}$, thus there was no storm.

Day 268-269 (25-26 September), 2001 (Fig. A15). A SYM$H=-118 \mathrm{nT}$ magnetic storm caused by a sheath upstream of an ICME. The shock at 2027 UT (2327 LT) triggered a short-duration substorm of $S M L=-1544$ nT intensity and a below threshold GIC event. At the time of the shock Mäntsälä was in the midnight sector. A 20 A GIC was associated with an intense substorm spike of $S M L=-2264 \mathrm{nT}$ occurring in the storm main phase. The intense substorm was caused by a strong solar wind density spike of Nsw $=69 \mathrm{~cm}^{-3}$.

Days 294-295 (21-22 October), 2001 (Fig. A16). An ICME related $S Y M-H=-219 \mathrm{nT}$ magnetic storm. The strong shock at 1649 UT (1949 LT) created a sudden onset of a SML = $-1148 \mathrm{nT}$ substorm and a GIC below study threshold. At the time of the shock, Mäntsälä was in the dusk-midnight sector. In the storm main phase, an $11 \mathrm{~A}$ GIC is associated with a $S M L=$ -1824 nT short-duration substorm. A second larger 18 A GIC occurs within a long-duration $S M L=-1160 \mathrm{nT}$ substorm. Both the latter GIC and substorm onset occur at the same time as a Nsw $=65 \mathrm{~cm}^{-3}$ solar wind density spike. This occurred in the storm recovery phase. Two more GICs of $12 \mathrm{~A}$ and $13 \mathrm{~A}$ intensity occur in a multiple substorm period. There is no obvious 1-to-1 relationship between the GICs and the substorms for these two GIC events. A ICME filament is indicated by the vertical shading at $\sim 1200$ UT day 295 . There are some small

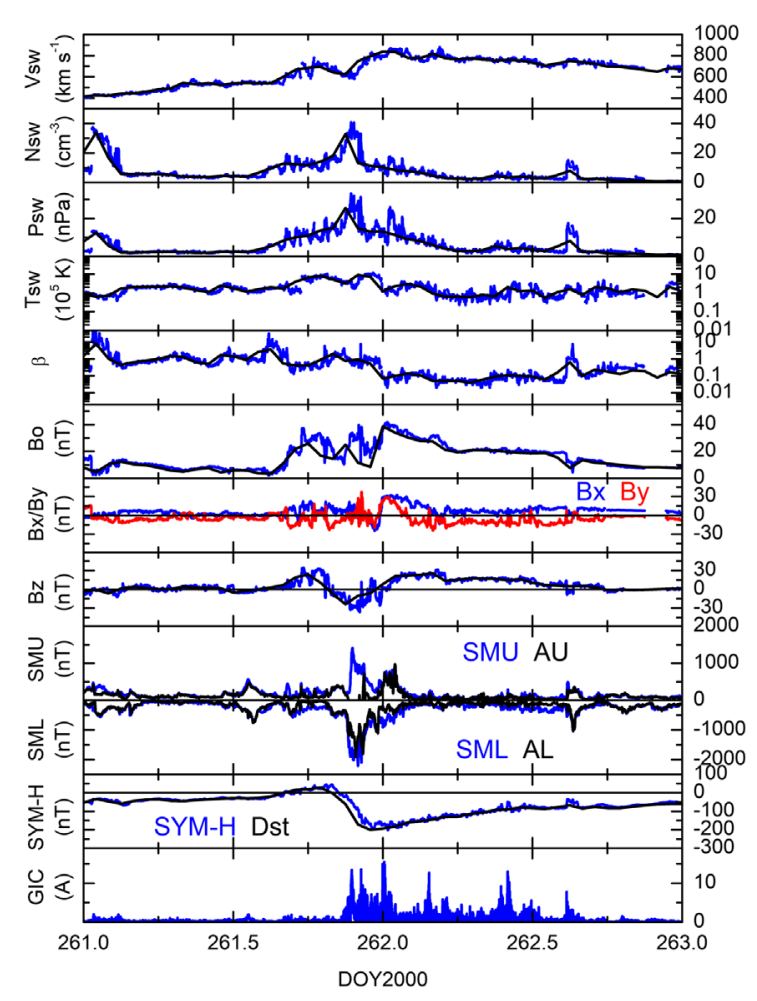

Fig. A7. Events on days 261-262 (17-18 September) 2000. Same format as in Figure A1.

GICs present during the interval but they are below the study threshold.

Day 310 (6 November), 2001 (Fig. A17). The sheath portion of an ICME (there is a data gap) causes a $S Y M-H=-320 \mathrm{nT}$ superstorm. Although the shock occurred in a data gap, a clear $\mathrm{SI}^{+}$of intensity $86 \mathrm{nT}$ at $\sim 0153 \mathrm{UT}(0453 \mathrm{LT})$ and a correlated 32 A GIC were present. Mäntsälä was in the dawn sector. The shock triggered a short-duration $S M L=-2301 \mathrm{nT}$ intense substorm. There were intense $S M L=\sim-2300 \mathrm{nT}$ substorm activities (following the initial intense substorm) with many GIC events with intensities up to $27 \mathrm{~A}$. In the storm recovery phase two SSSs of even greater intensities of $S M L=-2839$ nT and -2494 nT occurred, but these did not cause GICs above the study threshold.

Day 328 (24 November), 2001 (Fig. A18). A sheath upstream of an ICME caused a double dip magnetic storm of $S Y M-H=-233 \mathrm{nT}$ peak intensity. The MC had a northward IMF orientation and caused the recovery phase of the storm. The shock occurred at 0600 UT (0900 LT), when Mäntsälä was in the morning sector. The shock triggered a long-duration $S S S$ which peaked at $S M L=-3839$ nT hours later. Either the shock or the onset of the SSS caused a 21 A GIC. The SSS peak was correlated with a 32 A GIC. Later GICs of 25 A and 26 A were associated with the decay phase of the SSS. The SSS was triggered/enhanced in intensity by a Nsw $=65 \mathrm{~cm}^{-3}(\mathrm{PP})$ and an IMF Bn turning. The PP may be a coronal loop portion of the ICME. In the second dip of the storm, another SSS of $S M L=-3281 \mathrm{nT}$ peak intensity caused GICs of 12,10 and 11 A. There were $40>10$ A GICs in this day. 


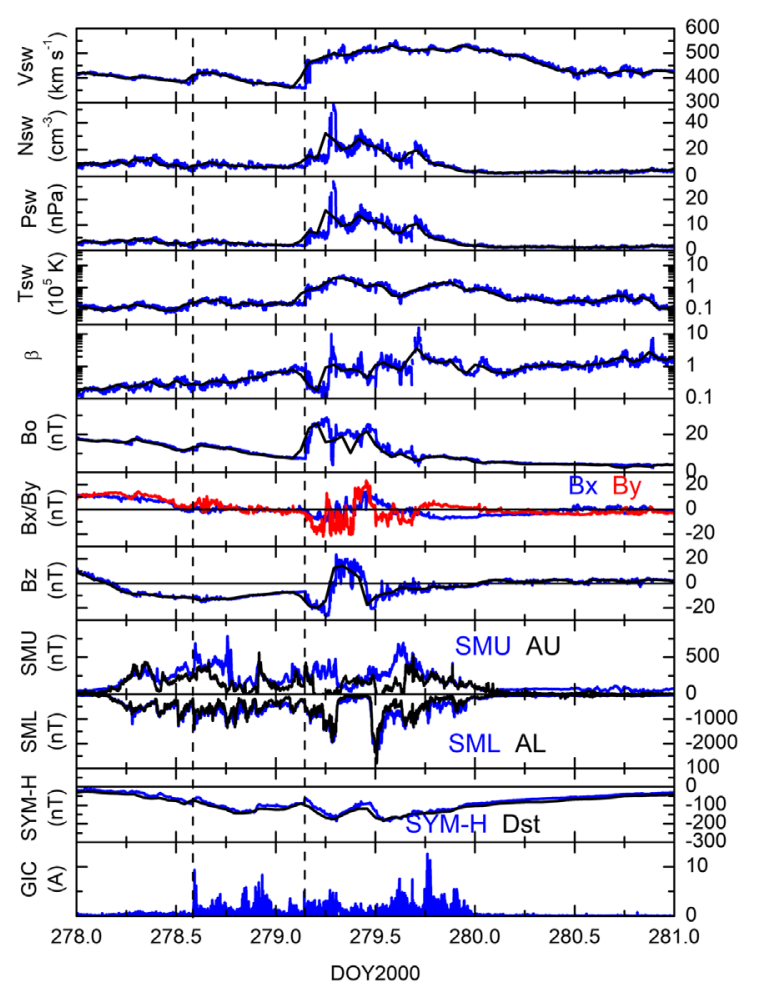

Fig. A8. Events on days 278-279 (4-5 October) 2000. Same format as in Figure A1.

Day 107 (17 April), 2002 (Fig. A19). A storm of SYM-H= $-100 \mathrm{nT}$ is caused by the sheath ahead of an ICME. The shock at 1107 UT (1407 LT) was time-coincident with a GIC of 19 A and a $S M L=-1523$ nT sharp onset substorm. Mäntsälä was in the afternoon sector at the time of the shock. There were no GICs of threshold intensities during the storm main phase.

Day 143 (May 23), 2002 (Fig. A20). A double dip magnetic storm with peak intensity $S Y M-H=-115 \mathrm{nT}$ was caused by a compound interplanetary event: shock/sheath, MC, and a possible filament. A Bs field in the MC caused the first storm dip and the following Bs interval at the onset of the high-density possible filament $\left(35 \mathrm{~cm}^{-3}\right)$ at $\sim 1546 \mathrm{UT}(1846 \mathrm{LT})$, indicated by the vertical red dashed line, caused the second storm dip. The shock at $\sim 1051$ UT $(1351 \mathrm{LT})$ created a $S M L=-662 \mathrm{nT}$ substorm and a $10 \mathrm{~A}$ GIC. The substorm is not detected in the $A L$ index nor the $S M L$ indices. It is noted by the $S M U$ index alone $(S M U=1726 \mathrm{nT})$. Mäntsälä was in the afternoon sector during this event. The filament Bs caused the second dip of the storm. The possible filament and its Bs triggered a $S M L=-1760 \mathrm{nT}$ long-duration substorm and many GICs, but not above the $10 \mathrm{~A}$ threshold of this study. It is possible that this substorm caused the second dip of the two-dip storm.

Days 250-251 (7-8 September), 2002 (Fig. A21). A double dip storm with peak intensity $S Y M-H=-168 \mathrm{nT}$ was caused by sheath Bs variations ahead of a MC. The shock at $\sim 1637$ UT (1937 LT) created a GIC but below the threshold of this study. Mäntsälä was in the dusk sector at the time. There were three clear GICs of 26, 21 and 24 A intensities. The first GIC is correlated with an IMF Bn turning ending the first dip storm. The $\mathrm{Bn}$ turning started the recovery of the first dip storm. There was

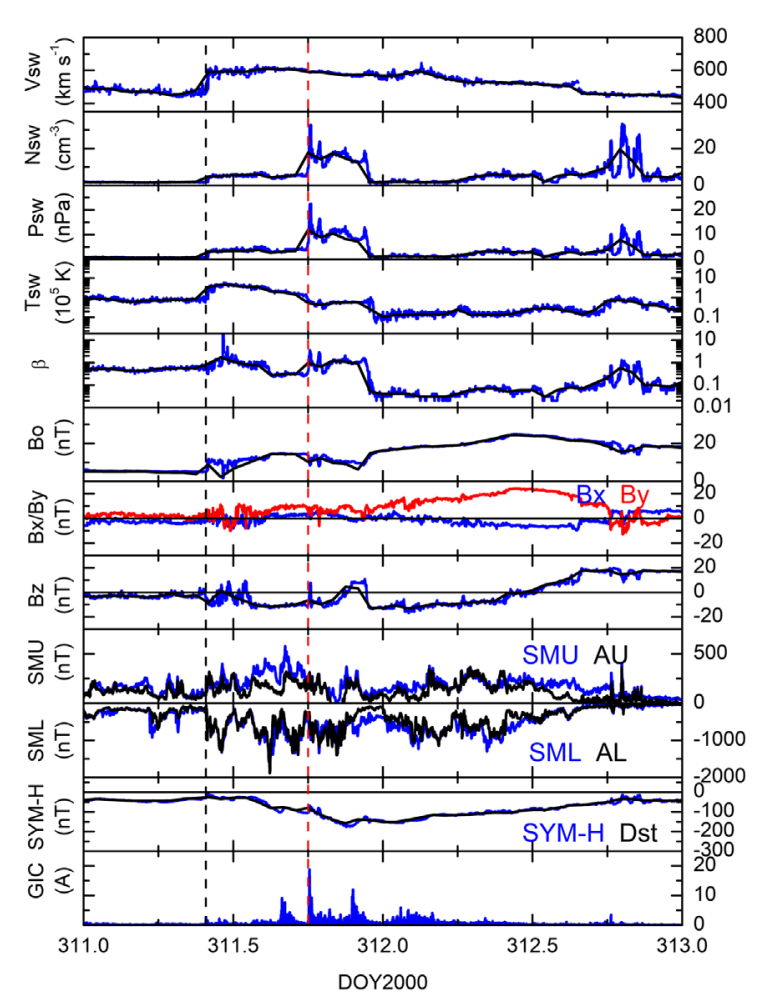

Fig. A9. Events on days 311-312 (6-7 November) 2000. Same format as in Figure A1. The vertical red dashed line indicates a PP event.

no substorm associated with the GIC. The second GIC had no obvious substorm or interplanetary related features. The third GIC occurred in the beginning of the storm recovery phase and was associated with a substorm of $S M L=-1116 \mathrm{nT}$ intensity.

Days 274-275 (1-2 October), 2002 (Fig. A22). This event was previously discussed in Results section (Fig. 8). A lowspeed solar wind at Vsw $\sim 420 \mathrm{~km} \mathrm{~s}^{-1}$ with a long-period Bz wave with Bs reaching $23 \mathrm{nT}$ caused a $S Y M-H=-153 \mathrm{nT}$ magnetic storm (peak at 1253 UT day 274). There was geomagnetic auroral zone activity in this low-speed solar wind. A $S M L=-1906 \mathrm{nT}$ short-duration substorm spike caused a double GIC of $28 \mathrm{~A}$ and $19 \mathrm{~A}$ intensities. The GICs occurred in the beginning of the storm recovery phase. There is no obvious interplanetary cause of this substorm.

Day 297 (24 October), 2002 (Fig. A23). A high-speed stream of Vsw $\sim 800 \mathrm{~km} \mathrm{~s}^{-1}$ peak speed caused a CIR and HILDCAA-like interval. The Bs in the CIR caused a day-long $S Y M-H=-88$ nT magnetic storm. There were no shocks. The CIR double Bs feature caused two long-duration substorms with peak intensities of $S M L=-1306 \mathrm{nT}$ and $-1112 \mathrm{nT}$. Neither substorm caused a GIC of intensity over the study level. A double GIC of 19 A and 14 A were associated with a much smaller substorm of $S M L=-993 \mathrm{nT}$ intensity.

Days 149-150 (29-30 May), 2003 (Fig. A24). A double shock/sheath event. The second sheath Bs caused a $S Y M-H=$ -164 nT magnetic storm. The MC had a Bn field and caused the storm recovery phase. The first shock at $\sim 1226$ UT 


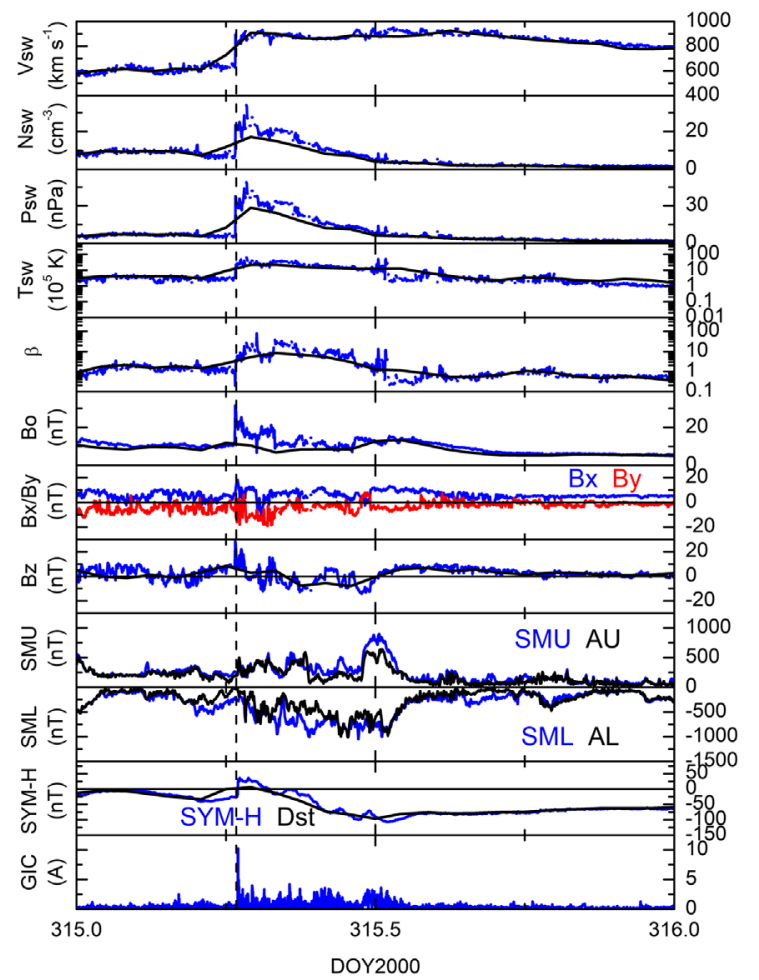

Fig. A10. Events on day 315 (10 November) 2000. Same format as in Figure A1.

(1526 LT) did not trigger a substorm nor a GIC of study level intensity. The second shock occurred at 1913 UT (2213 LT) and triggered an intense substorm of SML intensity $-2461 \mathrm{nT}$. The shock-related GIC did not reach the study threshold. Mäntsälä was in the afternoon sector during the first shock and in the dusk-midnight sector during the second shock. A cluster of GICs with peaks of $11 \mathrm{~A}, 11 \mathrm{~A}, 11 \mathrm{~A}$ and $12 \mathrm{~A}$ occurred in the storm main phase but there are no obvious 1-to-1 correlations with substorm peaks.

Day 287 (14 October), 2003 (Fig. A25). CIR Bs fields caused a double dip magnetic storm of $S Y M-H=-103 \mathrm{nT}$ intensity. There was no shock. The CIR double dip wave in $\mathrm{Bz}$ causing two long-duration substorms with peak intensities of $S M L=-1447 \mathrm{nT}$ and $-1385 \mathrm{nT}$. The two substorms caused the double dip magnetic storm. The first substorm peak causes a GIC of 29 A. The second substorm caused GICs, but below the study threshold. The high-speed stream proper had a peak speed of $750 \mathrm{~km} \mathrm{~s}^{-1}$.

Days 302-303 (29-30 October), 2003 (Fig. A26). This event was previously discussed in Results section (Fig. 2). A sheath and MC Bs caused the first October Halloween superstorm of intensity $S Y M-H=-390 \mathrm{nT}$. This was a double dip magnetic storm with the sheath causing the first dip and a MC Bs causing the second dip. The strong shock at $\sim 0612$ UT (0912 LT) and triggered the sharp onset of a long duration, two-peak SSS of $S M L=-3177 \mathrm{nT}$ (at 0624 UT) and $-3548 \mathrm{nT}$ (at 0648 UT) intensity and a cluster of GICs of 33 A (at 0646 UT), $57 \mathrm{~A}$ (at $0657 \mathrm{UT}$ ), $51 \mathrm{~A}$ (at $0703 \mathrm{UT}$ ) and $52 \mathrm{~A}$ (at 0727 UT) later in the event. Either the shock or the onset of the SSS caused a GIC of 25 A. Mäntsälä was in the morning sector at

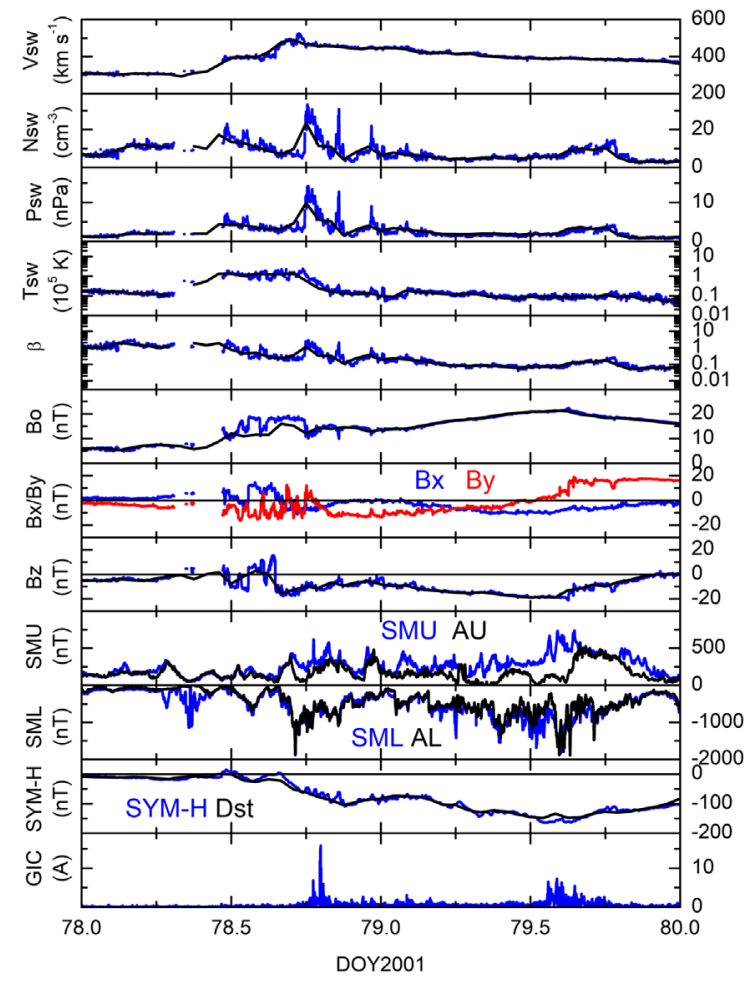

Fig. A11. Events on day 78 (19 March) 2001. Same format as in Figure A1.

the time of the largest GICs. There are many GICs $>10 \mathrm{~A}$ in the two main phases of this double dip storm. However, the most intense events occurred in the first SSS immediately after the shock. There is a GIC of 30 A at 2230 UT (0130 LT) day 302 at the start of the second dip storm. This is associated with an intense storm of $S M L=-2340 \mathrm{nT}$. There is a $36 \mathrm{~A} \mathrm{GIC} \mathrm{at}$ 0110 UT (0410 LT) day 303 at the start of the recovery phase of the second dip storm.

Days 303-304 (30-31 October), 2003 (Fig. A27). This event was previously discussed in Results section (Fig. 3). The second "Halloween" superstorm. A sheath Bs upstream of an ICME causes the second October Halloween storm of intensity $S Y M-H=-432 \mathrm{nT}$. A solar wind density spike (PP) at $\sim 1949$ UT (2249 LT) (denoted by the $\mathrm{SI}^{+}$of $\sim 61 \mathrm{nT}$ ) created a GIC of 49 A and a short-duration SSS of amplitude $S M L=$ -3872 nT. Mäntsälä was in the midnight sector at the time. A second short-duration $S M L=-2724 \mathrm{nT} S S S$ occurred in the storm main phase. It is associated with a double GIC of 33 and 27 A. There are two clusters of GICs with > 10 A intensities in the storm recovery phase. They are associated with substorm intervals of peak intensities of $S M L=-1821$ and -797 $\mathrm{nT}$, respectively. In the first cluster there is a GIC of $30 \mathrm{~A}$ at 0213 UT on day 304. In the second cluster there is a $27 \mathrm{~A}$ GIC at 0536 UT day 304. There is a fourth GIC cluster with $16 \mathrm{~A}$ at $1119 \mathrm{UT}, 19 \mathrm{~A}$ at $1152 \mathrm{UT}, 16 \mathrm{~A}$ at $1119 \mathrm{UT}, 14 \mathrm{~A}$ at $1227 \mathrm{UT}$ and $16 \mathrm{~A}$ at $1246 \mathrm{UT}$. There were $90 \mathrm{GICs}>10$ $\mathrm{A}$ and $3 \mathrm{GICs}>30 \mathrm{~A}$.

Days 324-325 (20-21 November), 2003 (Fig. A28). The third "Halloween" superstorm. The November 2003 Halloween storm of $S Y M-H=-490 \mathrm{nT}$ intensity was caused by Bs in the 


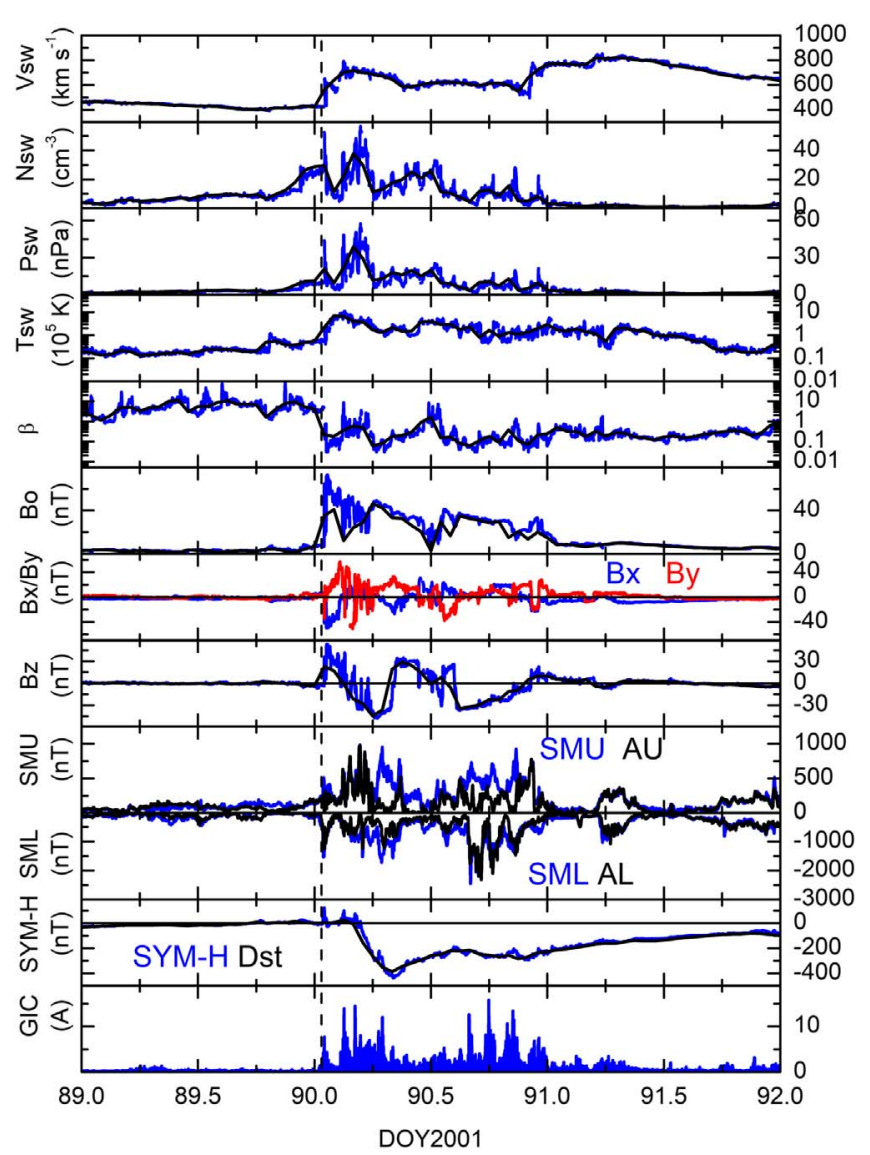

Fig. A12. Events on day 90 (31 March) 2001. Same format as in Figure A1.

sheath, Bs in an ICME MC followed by a possible filament. The shock at $\sim 0803$ UT (1103 LT) only created a small GIC below the study threshold and a $S M L=-2031 \mathrm{nT}$ short-duration sharp onset intense substorm. Mäntsälä was in the noon sector at the time of the shock. In the MC generated storm main phase, there are 4 large GICs. A short-duration substorm detected only in SMU (912 nT) is coincident with the first GIC of $23 \mathrm{~A}$ at 1408 UT. The next two GICs of 19 A (at 1518 UT) and 24 A (at $1640 \mathrm{UT}$ ) are not related to any obvious substorm features. A $S S S$ of $S M L=-4141 \mathrm{nT}$ intensity at 1634 UT caused the largest (double) GIC of 19 A and 24 A. These may be associated with a sharp solar wind density spike of $25 \mathrm{~cm}^{-3}$. The density spike is the onset of a possible ICME filament. There is a $16 \mathrm{~A}$ GIC in the storm recovery phase caused by the last, isolated substorm of amplitude $S M L=-1714 \mathrm{nT}$.

Day 326 (22 November), 2003 (Fig. A29). This interval was a HSS with a relatively constant Vsw $\sim 540-640 \mathrm{~km} \mathrm{~s}^{-1}$ speed. The IMF Bs peak of $-9.2 \mathrm{nT}$ generated a magnetic storm of $S Y M-H=-97 \mathrm{nT}$. There was no obvious shock and/or CIR. In the first Bs interval there is a cluster of substorms with SML peaks all of - $1500 \mathrm{nT}$ intensities. One large GIC of $14 \mathrm{~A}$ is associated with one short-duration substorm of intensity $S M L=-1561 \mathrm{nT}$. This substorm cannot be detected in the $A L$ index. It is most remarkable in the $S M U$ index. The other substorms with the same intensities did not generate GICs above the study threshold.

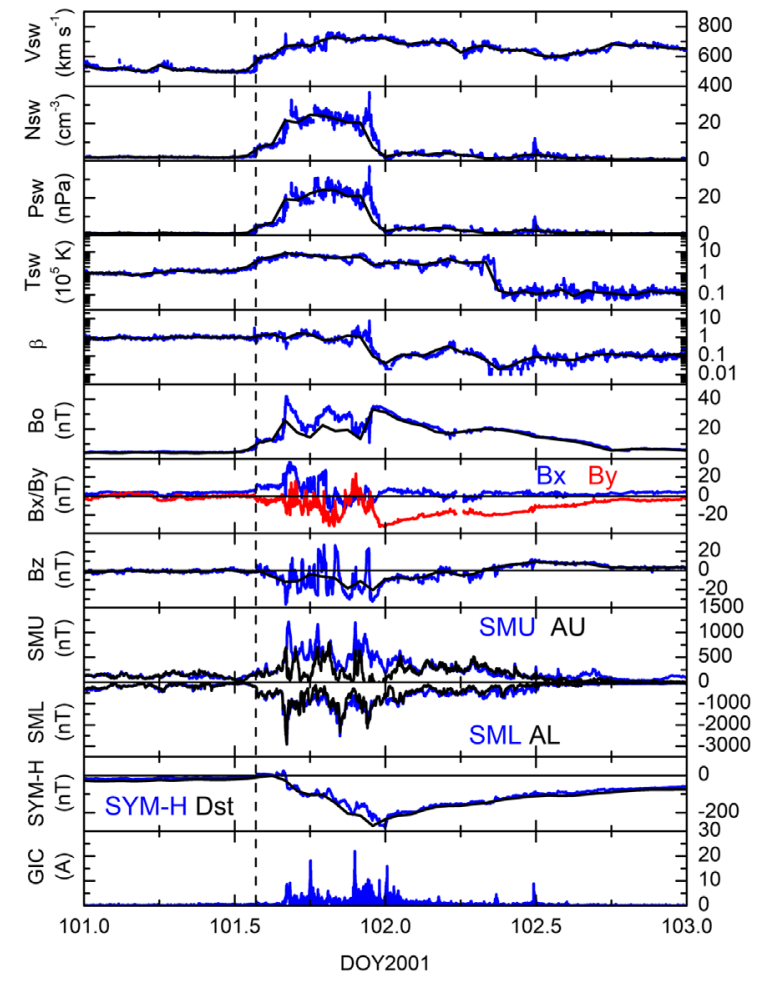

Fig. A13. Events on days 101-102 (11-12 April) 2001. Same format as in Figure A1.

Day 344 (10 December), 2003 (Fig. A30). A pure HSS event with a peak speed of $\mathrm{Vsw}=840 \mathrm{~km} \mathrm{~s}^{-1}$ caused a magnetic storm of $S Y M-H=-71 \mathrm{nT}$ intensity. Alfvénic Bs fluctuations caused a HILDCAA interval with lots of substorms, but no SSSs. One isolated 11 A GIC is caused by a substorm with $S M L$ peak intensity of $-1830 \mathrm{nT}$. This substorm is not detected in the $A L$ index.

Day 94 (3 April), 2004 (Fig. A31). A moderate speed ICME whose upstream sheath/pileup region caused a double dip magnetic storm of $S Y M-H=-149 \mathrm{nT}$ intensity. The peak speed of the ICME is only $496 \mathrm{~km} \mathrm{~s}^{-1}$ in a background Vsw $\sim 400 \mathrm{~km} \mathrm{~s}^{-1}$. The storm double dip is caused by a long $B z$ wave giving 2 intervals of $\mathrm{Bs}$ in the sheath ahead of the MC. The MC has a Bn field and leads to the storm recovery phase. The shock at 1413 UT (1713 LT) did not cause a GIC with intensity above study level. At the time Mäntsälä was in the dusk sector. One GIC of 11 A occurred at the cusp between the first dip storm and the second dip storm. There was no obvious substorm or solar wind feature associated with this GIC.

Days 312-313 (7-8 November), 2004 (Fig. A32). A triple $\operatorname{dip} S Y M-H=-382 \mathrm{nT}$ superstorm caused by a sheath Bs interval and a MC Bs interval. There were three shocks and sheaths in this interplanetary event but the first two shocks were not geoeffective. Both the first shock at the beginning of day 312 and the second shock at $\sim 1056$ UT (1356 LT) day 312 created below threshold GIC events. The third shock at 1829 UT (2129 LT) was time-coincident with a 11 A GIC event. Mäntsälä was in the dusk sector during the second shock and in the dusk-midnight sector during the third shock. There are 


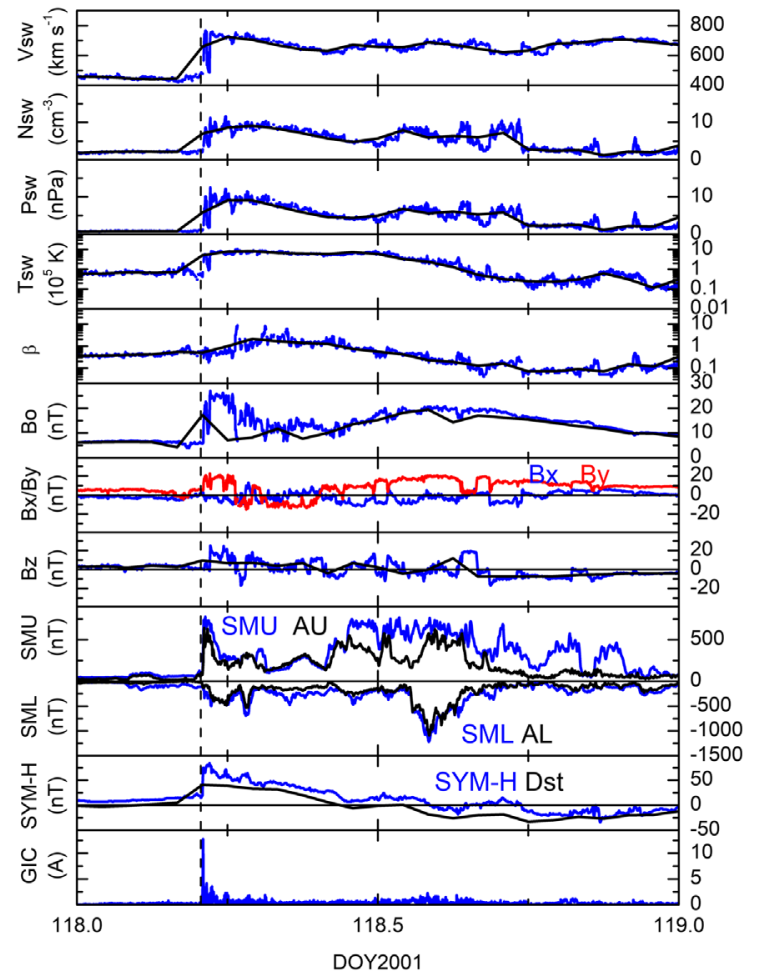

Fig. A14. Events on day 118 (28 April) 2001. Same format as in Figure A1.

two GIC event clusters occurring in the storm main phase. The largest GIC event of $35 \mathrm{~A}$ occurred in the first cluster during an intense substorm of $S M L$ amplitude $-2071 \mathrm{nT}$. The intense substorm was triggered by a solar wind PP and a sharp Bs intensification. The PP had a peak density of $\sim 29 \mathrm{~cm}^{-3}$. It could possibly a loop portion of the ICME. The second GIC cluster had many GICs $>10$ A (peak of 29 A) and occurred near third dip storm maximum. These individual GICs within the clusters do not appear to have one-to-one associations with substorm peak intensities. The GICs appear to be terminated by a reverse shock which decreased the IMF Bs. There were $38>10$ A GIC events during these two days.

Day 314 (9 November), 2004 (Fig. A33). A shock/sheath, shock/sheath and MC compound event causing a triple dip superstorm with intensities of $-139 \mathrm{nT},-271 \mathrm{nT}$ and -282 $\mathrm{nT}$, respectively. The first dip storm is caused by the first sheath $\mathrm{Bs}$, the second dip storm by the second sheath Bs and the third storm by the MC Bs. The first shock at $\sim 0932$ UT (1232 LT) triggered a GIC below the study threshold. The second shock at $\sim 1850$ UT ( 2150 LT) also did not trigger a GIC above the study threshold. At the time of these two shocks, Mäntsälä was in the noon sector and dusk-midnight sectors, respectively. The second shock triggered an intense substorm which reached a peak value of $S M L=-2264 \mathrm{nT}$ hours later. The substorm was due to sheath Bs which caused the second dip storm main phase. The second storm has a peak intensity of $S Y M-H=$ $271 \mathrm{nT}$ at 2103 UT day 314. This substorm onset is caused by a sharp Bs intensification associated with the shock compression of preexisting Bs fields. This intense substorm caused a

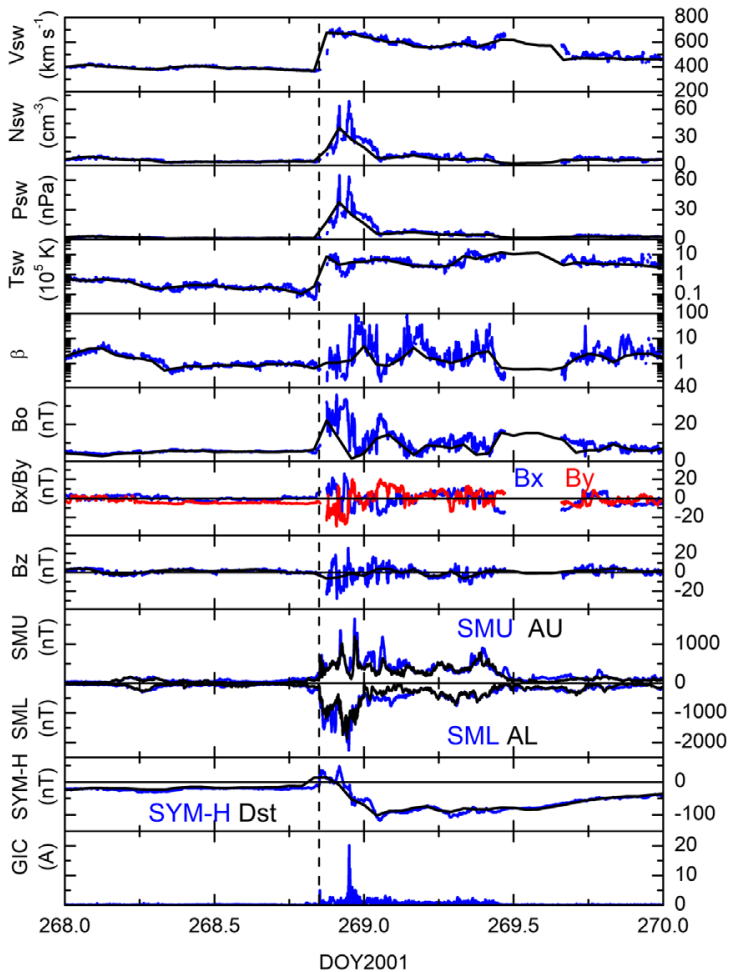

Fig. A15. Events on days 268-269 (25-26 September) 2001. Same format as in Figure A1.

cluster of three GICs with 43,42 and 21 A intensities. There were 20 GIC $>10$ A intensities during this day.

Day 21 (21 January), 2005 (Fig. A34). This event was previously discussed in Results section (Fig. 6). An ICME preceded by a strong shock/sheath. The sheath caused a storm of $S Y M-H=-101 \mathrm{nT}$. The shock at $\sim 1712$ UT (2012 LT) was time-coincident with a GIC of $13 \mathrm{~A}$ and triggered a sharp onset of a SSS which reached a peak value of $S M L=-4418 \mathrm{nT}$. Mäntsälä was in the dusk-midnight sector at the time. During the SSS decay phase there is a cluster of GICs reaching $27 \mathrm{~A}$. The GICs and substorm SMU intensification are associated with an unusual sharp interplanetary density spike up to Nsw $=55 \mathrm{~cm}^{-3}$. There is another GIC of intensity $22 \mathrm{~A}$ at 2015 UT which is time-coincident with a sharp solar wind density spike to $42 \mathrm{~cm}^{-3}$. There is no obvious substorm feature associated with this GIC. There were 12 GICs with $>10 \mathrm{~A}$ in this event.

Day 236 (24 August), 2005 (Fig. A35). A shock/sheath event ahead of an ICME event where the sheath Bs causes a magnetic storm of intensity $S Y M-H=-179 \mathrm{nT}$. The shock at 0614 UT (0914 LT) caused a GIC below the study threshold. Mäntsälä was in the morning sector at the time of the shock. In the storm main phase there are two SSSs with $S M L=-4046 \mathrm{nT}$ and $-3895 \mathrm{nT}$ peak intensities. There are no GICs above threshold associated with these events. There is a cluster of GICs with peak intensity of 13 A that occur in the SSS decay phase. This is at the end of the storm main phase and beginning of the recovery phase. There is no 1-to-1 correlation with the substorms. 


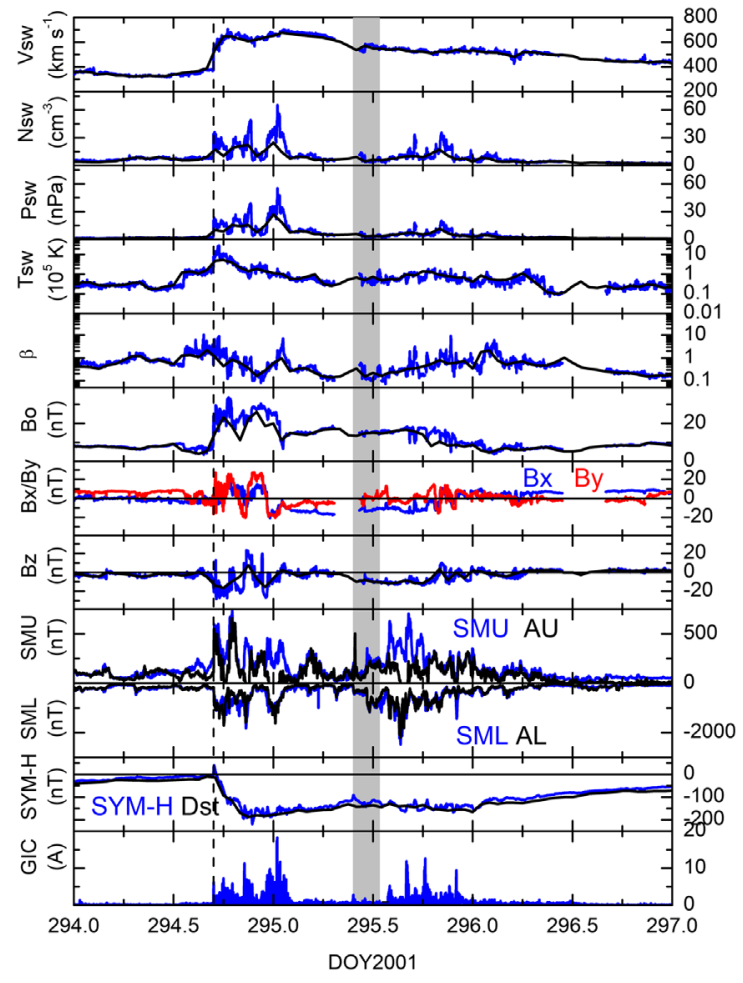

Fig. A16. Events on days 294-295 (21-22 October) 2001. Same format as in Figure A1. A ICME filament identified by Lepri \& Zurbuchen (2010) is indicated by the vertical shading.

Day 349 (15 December), 2006 (Fig. A36). An ICME event where the MC Bs caused a storm of $S Y M-H=-220 \mathrm{nT}$ intensity. The strong shock at $\sim 1414$ UT (1714 LT) triggered an intense substorm of $S M L=-2102 \mathrm{nT}$ intensity. Due to a GIC data gap, it is unknown whether there a GIC above study threshold or not. In the storm main phase there is an intense substorm of $S M L=-2264 \mathrm{nT}$. A GIC of $14 \mathrm{~A}$ at $0055 \mathrm{UT}$ is associated with the decay part of this substorm. There were three more GICs with $>10$ A intensities. They occurred at 0148 UT, 0224 UT and 0518 UT and have intensities of 13 A, $11 \mathrm{~A}$ and $12 \mathrm{~A}$, respectively. There are no obvious 1-to-1 GIC relationships with substorms.

Day 269 (26 September), 2011 (Fig. A37). A CIR and HSS event (maximum speed of Vsw $=734 \mathrm{~km} \mathrm{~s}^{-1}$ ) where the CIR Bs causes a storm of intensity $S Y M-H=-116 \mathrm{nT}$. The shock at $\sim 1236$ UT (1536 LT) triggers a short-duration substorm of intensity $S M L=-866 \mathrm{nT}$ but no GIC above study level. Mäntsälä was in the afternoon sector at the time of the shock. Two GICs of 12 and 16 A occurred in the storm main phase, but there are not obviously 1-to-1 associated with substorms. The first GIC occurs in the decay phase of an intense substorm of $S M L=-2006 \mathrm{nT}$ and the second more intense event occurs when the $S M L$ level is $<-500 \mathrm{nT}(S M L=-300 \mathrm{nT})$. The latter GIC is due to a solar wind plasma density spike. There is no associated substorm.

Day 58 (27 February), 2012 (Fig. A38). This event was previously discussed in Results section (Fig. 7). A Vsw $\sim 520 \mathrm{~km} \mathrm{~s}^{-1}$ slow ICME with no shock where the pileup Bs

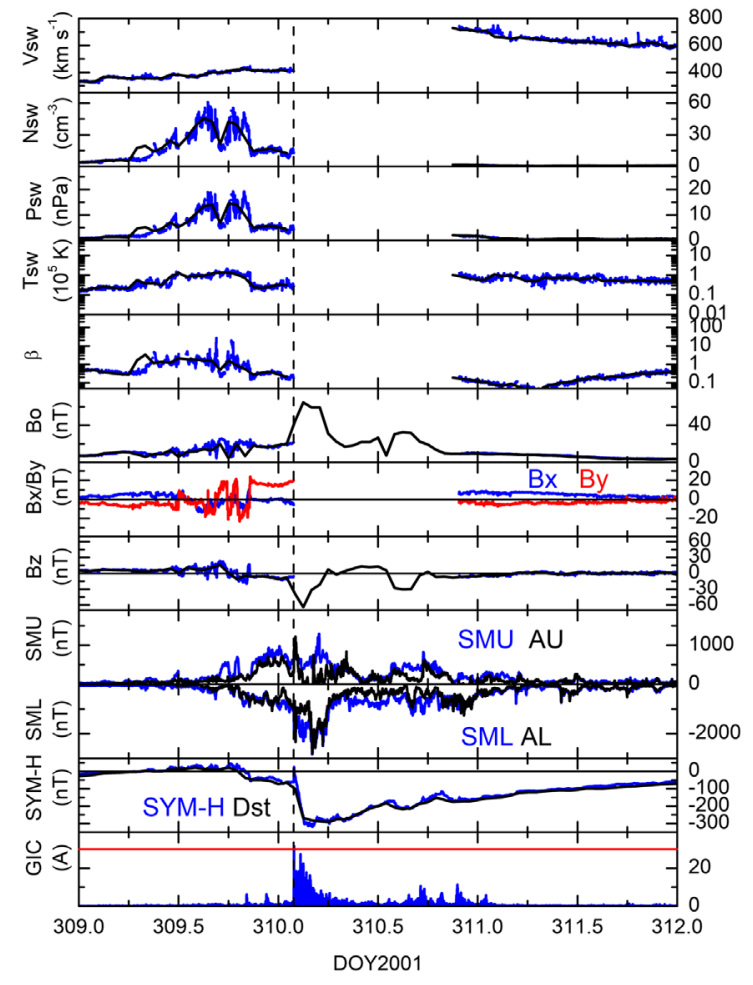

Fig. A17. Events on day 310 (6 November) 2001. Same format as in Figure A1.

fluctuations lead to a storm of $S Y M-H=-61 \mathrm{nT}$. The MC $\mathrm{Bz}=0 \mathrm{nT}$ causes the storm recovery phase. In this pileup region, there are moderate $S M L$ values with a peak of $-848 \mathrm{nT}$ but no intense GICs. A GIC event of $20 \mathrm{~A}$ is associated with an IMF Bn turning at the sheath/MC boundary. There is also a solar wind ram pressure decrease at that time. There are no substorms or GICs associated with the MC interval with IMF $\mathrm{Bn}$. A high plasma density region of $17.5 \mathrm{~cm}^{-3}$ follows the MC. This may be an ICME filament. The density causes a Bs interval and a substorm of $S M L=-1142 \mathrm{nT}$ at $0736 \mathrm{UT}$ (1036 LT) on day 59. The substorm only creates below threshold GICs.

Day 75 (15 March), 2012 (Fig. A39). The sheath Bs upstream of an ICME causes a magnetic storm of $S Y M-H=$ $-79 \mathrm{nT}$. The MC $B z=0 \mathrm{nT}$ leads to the storm recovery phase. The shock at 1307 UT (1607 LT) causes a GIC below the study threshold. Mäntsälä was in the afternoon sector at that time. A GIC of 39 A occurs at 1703 UT (2003 LT) on day 75 in main phase of the magnetic storm. There is a solar wind spike of density $12 \mathrm{~cm}^{-3}$ at 1704 UT that is the cause of the GIC.

Day 76 (17 March), 2013 (Fig. A40). An upstream sheath double Bs event caused a $S Y M-H=-131 \mathrm{nT}$ double dip magnetic storm. The shock at $~ 0601$ UT (0901 LT) triggered a $S M L=-958 \mathrm{nT}$ substorm but no GIC above the study limit. Mäntsälä was in the morning sector at the time of the shock. There is a cluster of 3 GICs of 32, 20 and 14 A intensities in the storm main phase near the storm peak. The GICs occurred on the decay phase of the substorm when the $S M L$ intensity was $\sim-990 \mathrm{nT}$. There were no obvious one-to-one relationships 


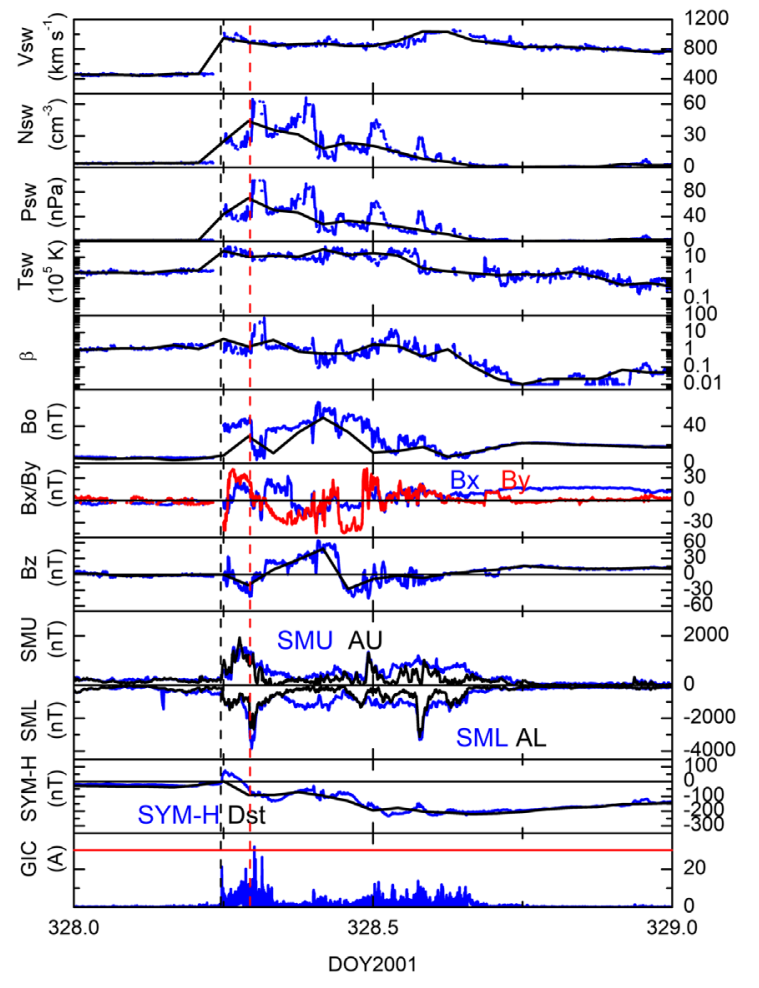

Fig. A18. Events on day 328 (24 November) 2001. Same format as in Figure A1. The GIC $=30 \mathrm{~A}$ level is shown by the horizontal red line in the GIC panel. The vertical red dashed line indicates a PP incidence.

between the GICs and substorm features. There were 7 GIC events with $>10 \mathrm{~A}$ in this day.

Day 275 (2 October), 2013 (Fig. A41). A magnetic storm of intensity $S Y M-H=-90 \mathrm{nT}$ caused by sheath Bs fields. The shock at $\sim 0156$ UT (0456 LT) triggered a moderate intensity substorm and no GIC above the study limit. The sheath Bs fields caused two substorms, the first of $S M L=-2017 \mathrm{nT}$ and a second of $S M L=-1963 \mathrm{nT}$. The substorms caused the storm main phase. The first substorm did not cause a GIC above the study limit. The second substorm causes a GIC of $15 \mathrm{~A}$ intensity.

Day 254 (11 September), 2015 (Fig. A42). A small Vsw = $\sim 640 \mathrm{~km} \mathrm{~s}^{-1} \mathrm{HSS}$, with presumably Bs (there is a data gap) that causes a $S Y M-H=-94 \mathrm{nT}$ double dip storm. A GIC double event of 16 A occurred in an interplanetary data gap at the start of the storm recovery phase. The GIC occurs at the end of substorm activity in the recovery phase of a $S M L=-1014 \mathrm{nT}$ substorm.

Day 280 (7 October), 2015 (Fig. A43). This is a double dip storm with intensities of $S Y M-H=-88 \mathrm{nT}$ and $-124 \mathrm{nT}$. The first storm was caused by small Bs in a Vsw $460 \mathrm{~km} \mathrm{~s}^{-1}$ slow solar wind. There were no major GICs associated with this event. The second storm is caused by CIR Bs (with no forward shock) ahead of a HSS of $\sim 650 \mathrm{~km} \mathrm{~s}^{-1}$. There were two small clusters of GICs. The first had a peak intensity of $21 \mathrm{~A}$ and was associated with a substorm of $S M L=-1573 \mathrm{nT}$ intensity. The second cluster reached $17 \mathrm{~A}$ and were not associated with

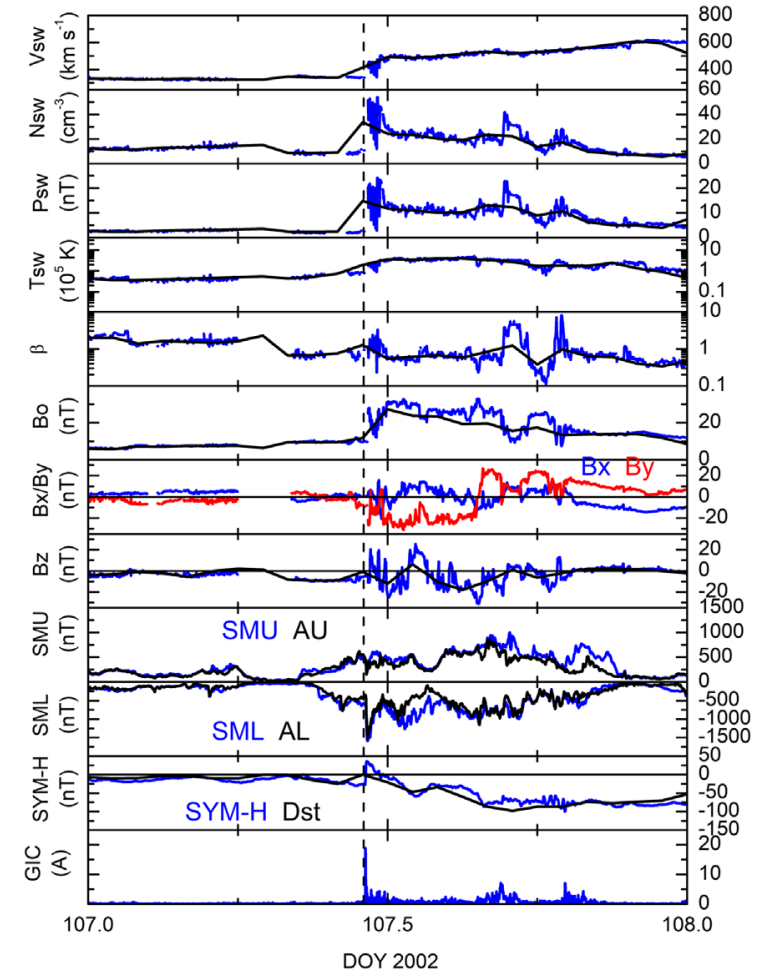

Fig. A19. Events on day 107 (17 April) 2002. Same format as in Figure A1.

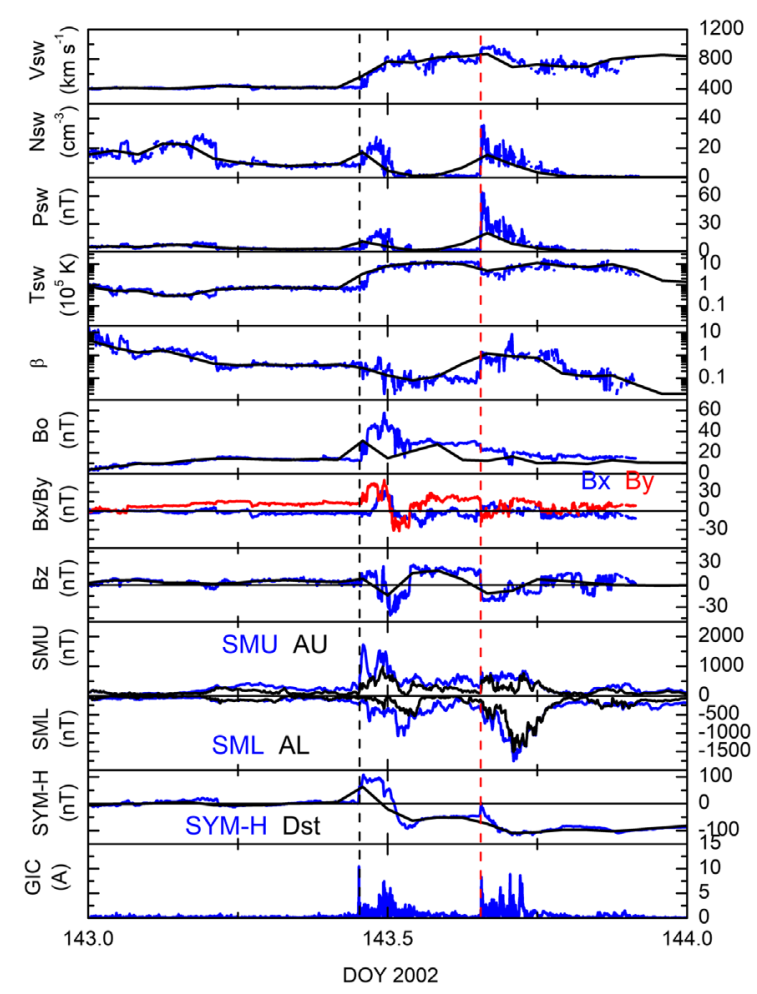

Fig. A20. Events on day 143 (23 May) 2002. Same format as in Figure A1. The vertical red dashed line indicates a possible filament. 


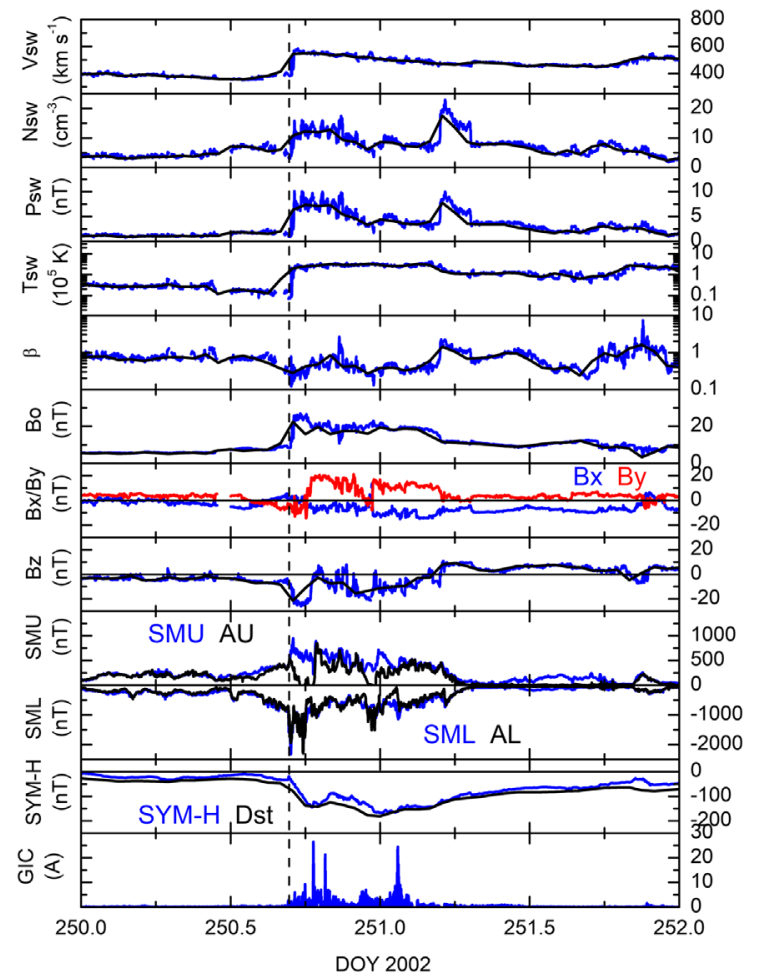

Fig. A21. Events on days 250-251 (7-8 September) 2002. Same format as in Figure A1.

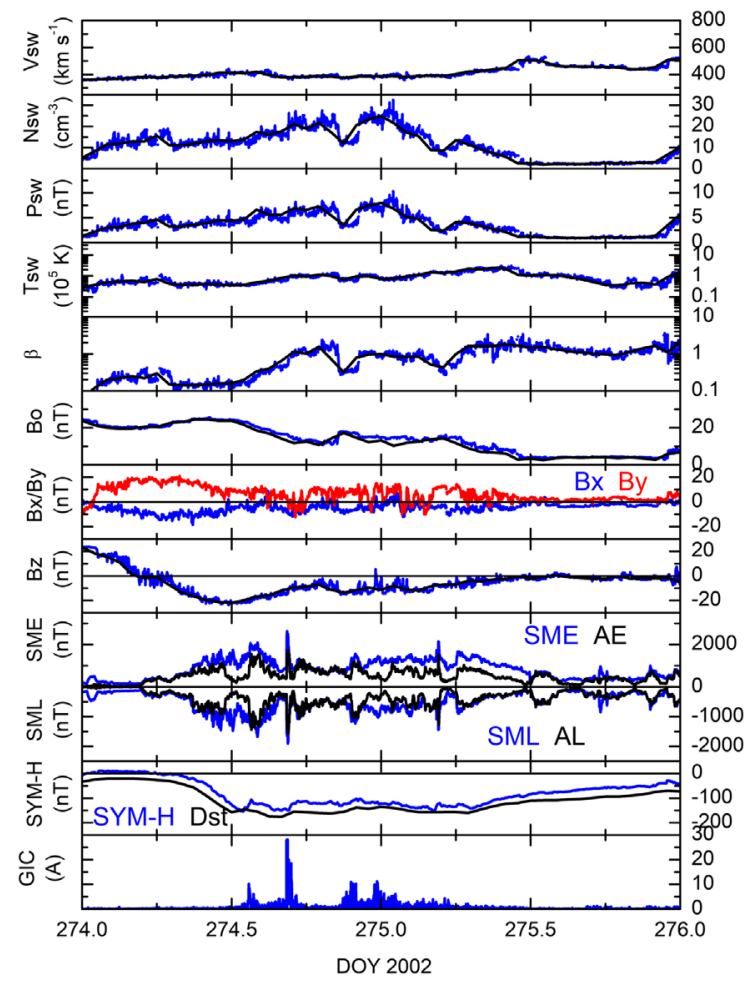

Fig. A22. Events on days 274-275 (1-2 October) 2002. Same format as in Figure A1.

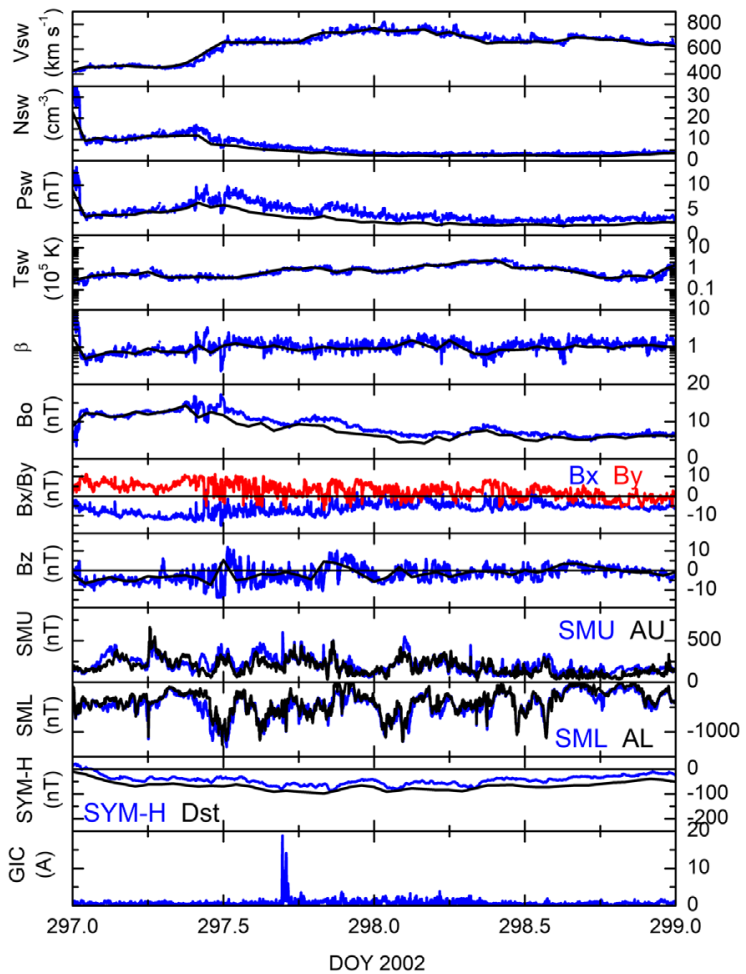

Fig. A23. Events on day 297 (24 October) 2002. Same format as in Figure A1.

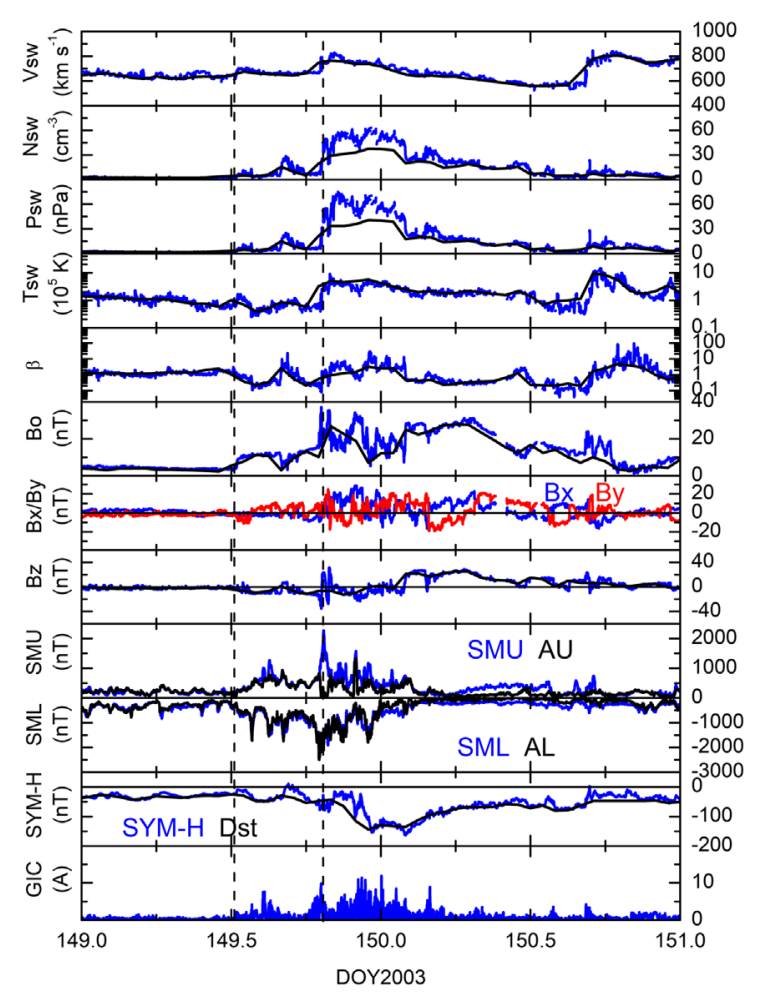

Fig. A24. Events on days 149-150 (29-30 May) 2003. Same format as in Figure A1. 


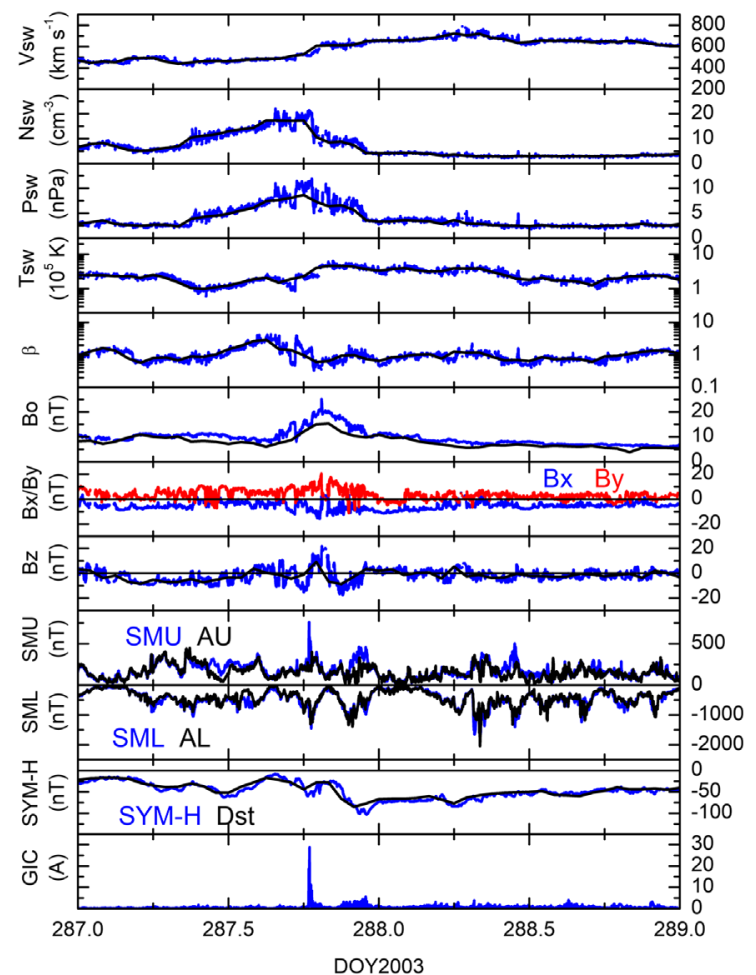

Fig. A25. Events on day 287 (14 October) 2003. Same format as in Figure A1.

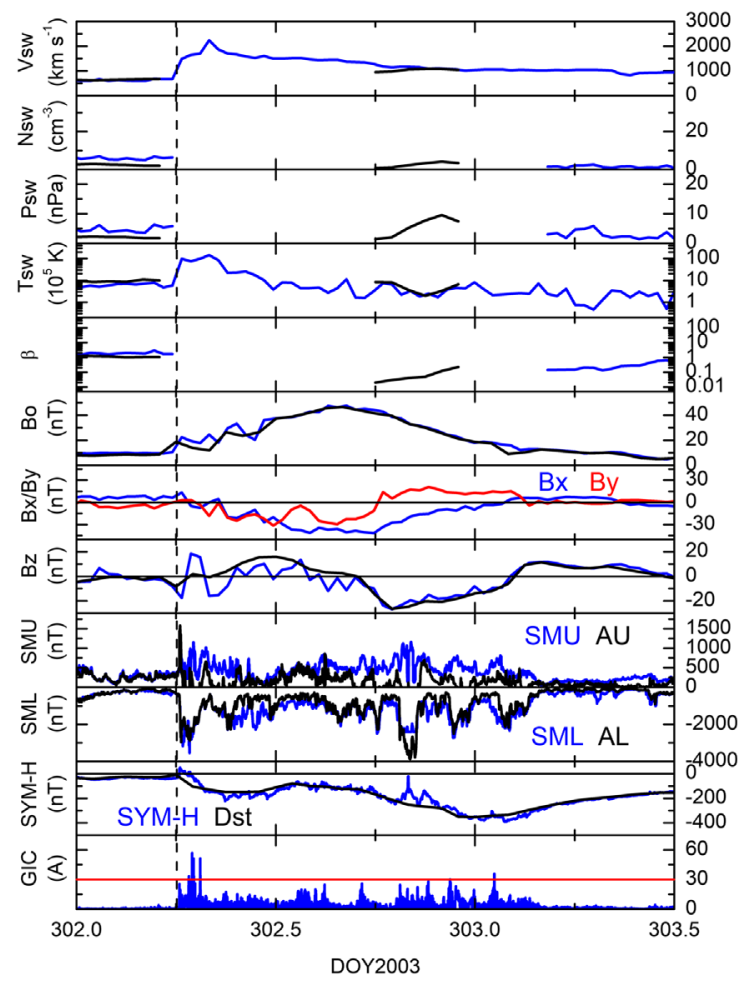

Fig. A26. Events on days 302-303 (29-30 October) 2003. Same format as in Figure A1. The red horizontal line in the GIC panel indicates the GIC $=30$ A level.

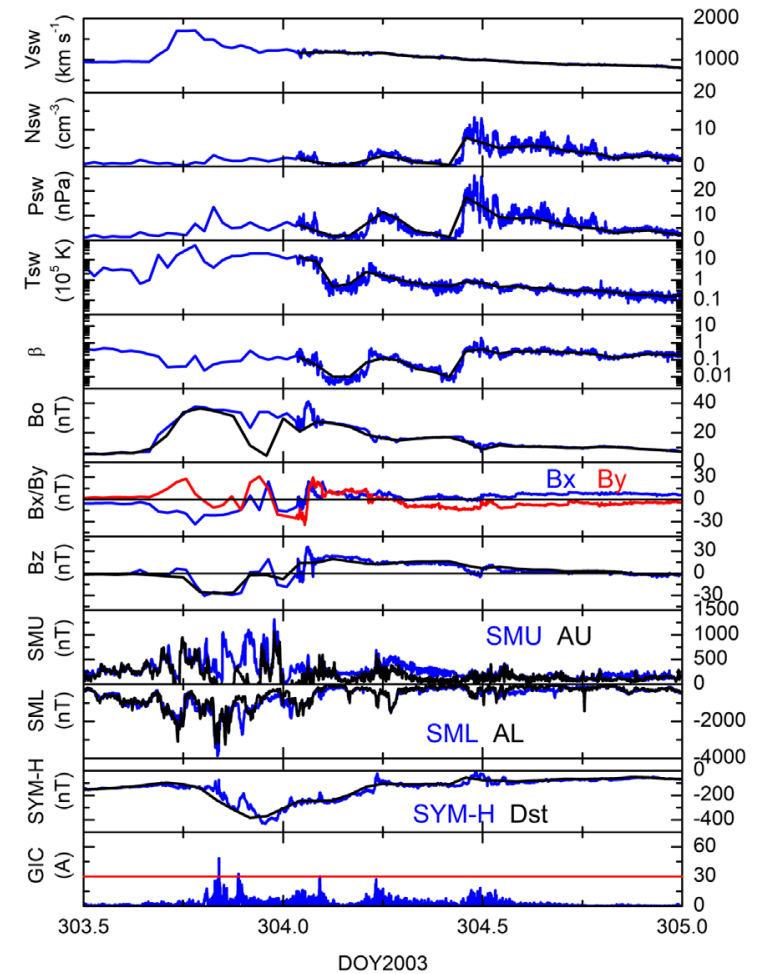

Fig. A27. Events on days 303-304 (30-31 October) 2003. Same format as in Figure A1. The red horizontal line in the GIC panel indicates the GIC $=30$ A level.

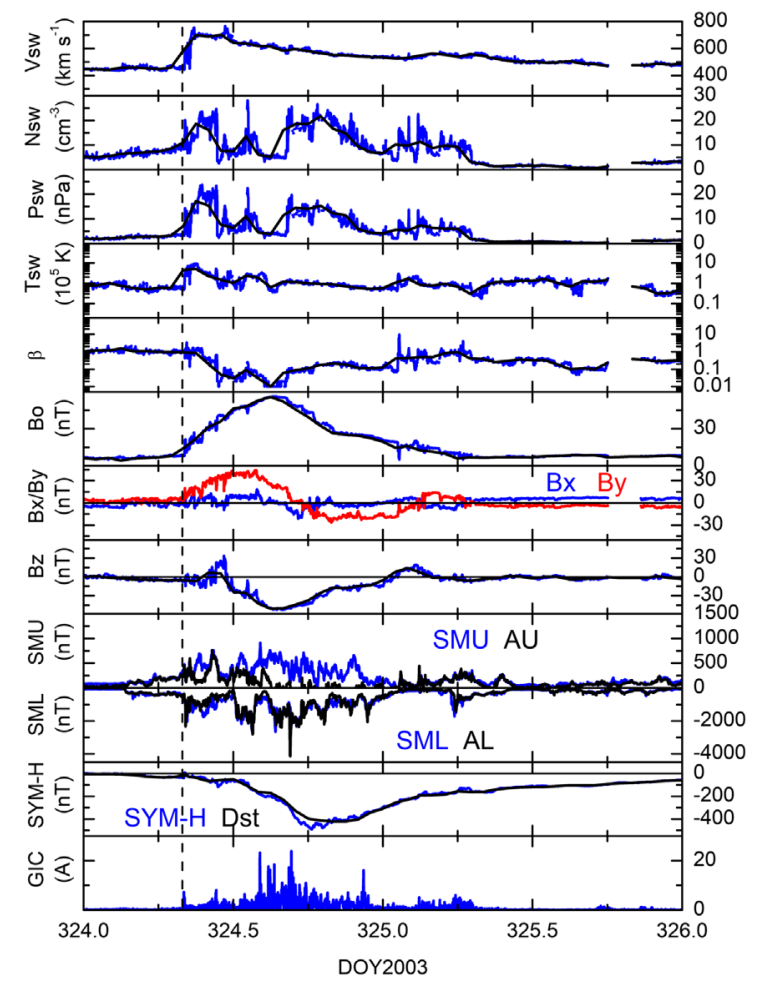

Fig. A28. Events on days 324-325 (20-21 November) 2003. Same format as in Figure A1. 


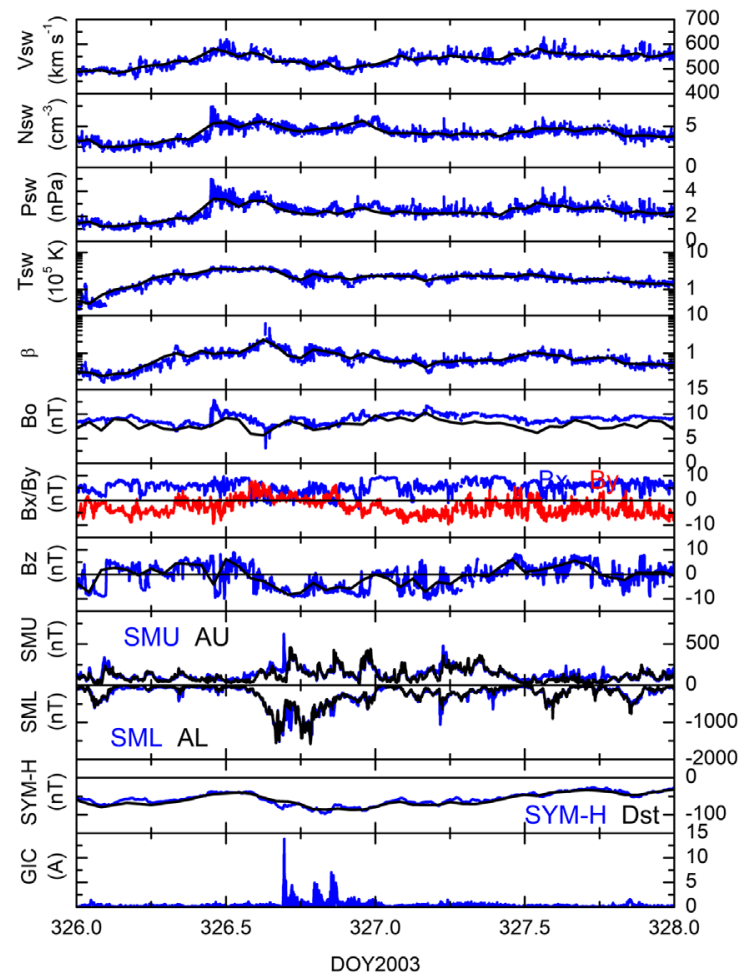

Fig. A29. Events on day 326 (22 November) 2003. Same format as in Figure A1.

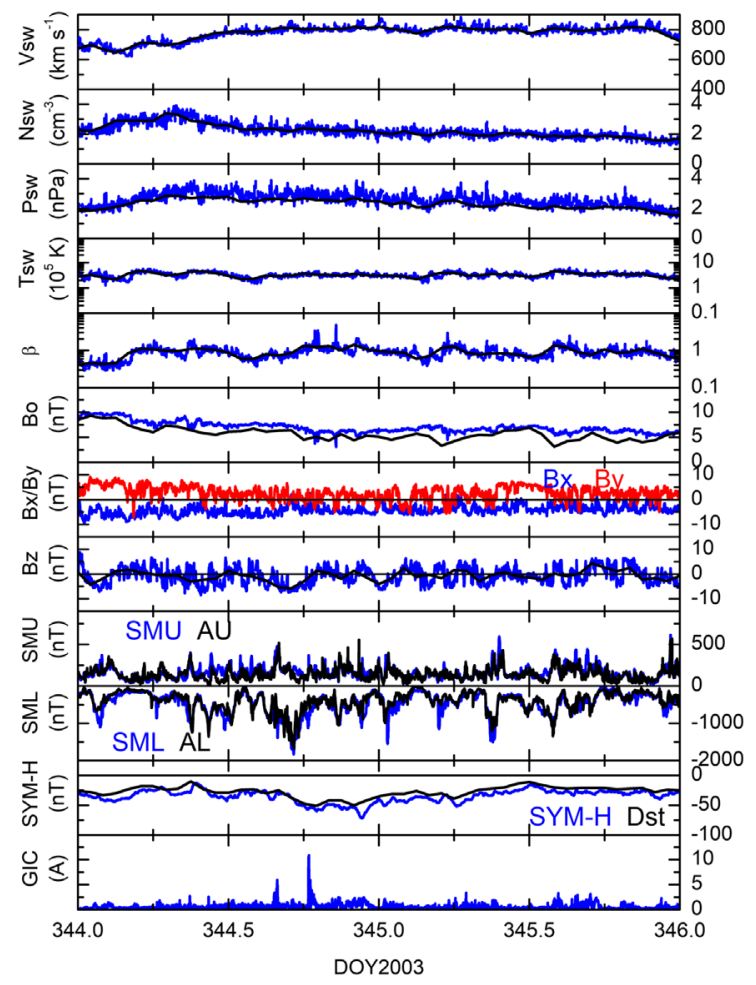

Fig. A30. Events on day 344 (10 December) 2003. Same format as in Figure A1.

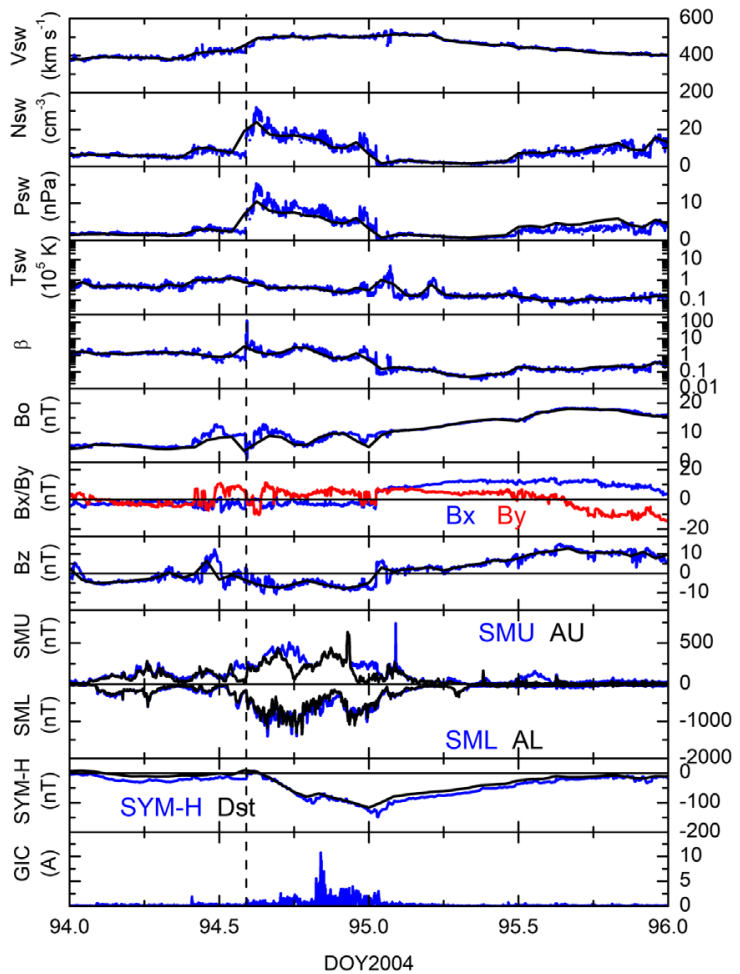

Fig. A31. Events on day 94 (3 April) 2004. Same format as in Figure A1.

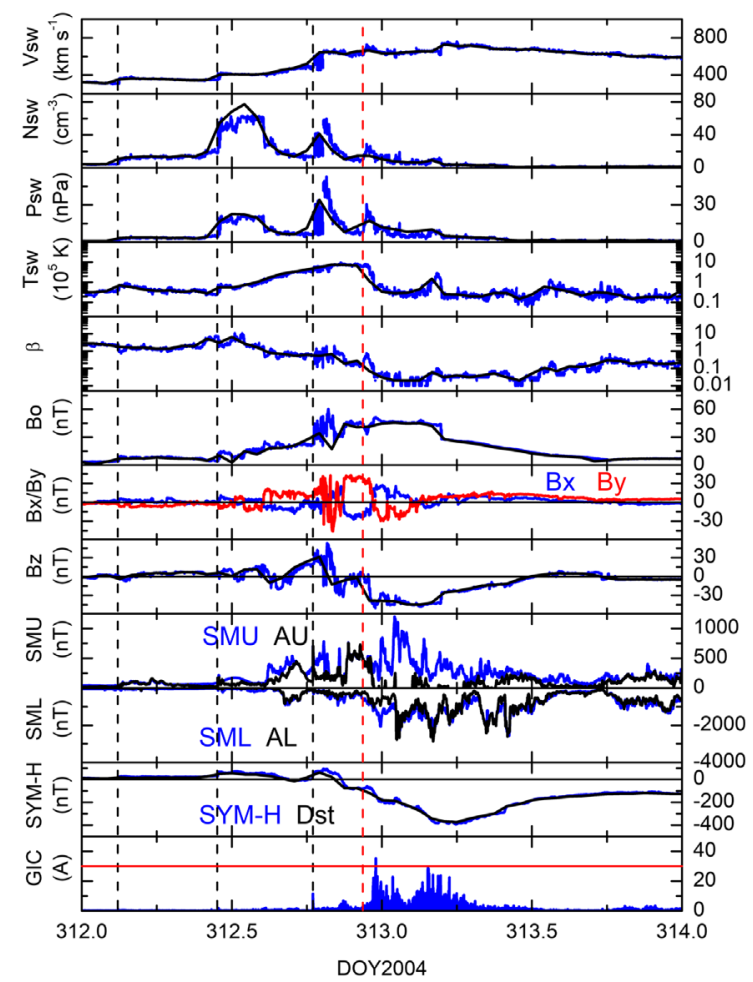

Fig. A32. Events on days 312-313 (7-8 November) 2004. Same format as in Figure A1. The horizontal red line in the GIC panel indicates the GIC $=30 \mathrm{~A}$ level. The vertical red dashed line indicates a PP incidence. 


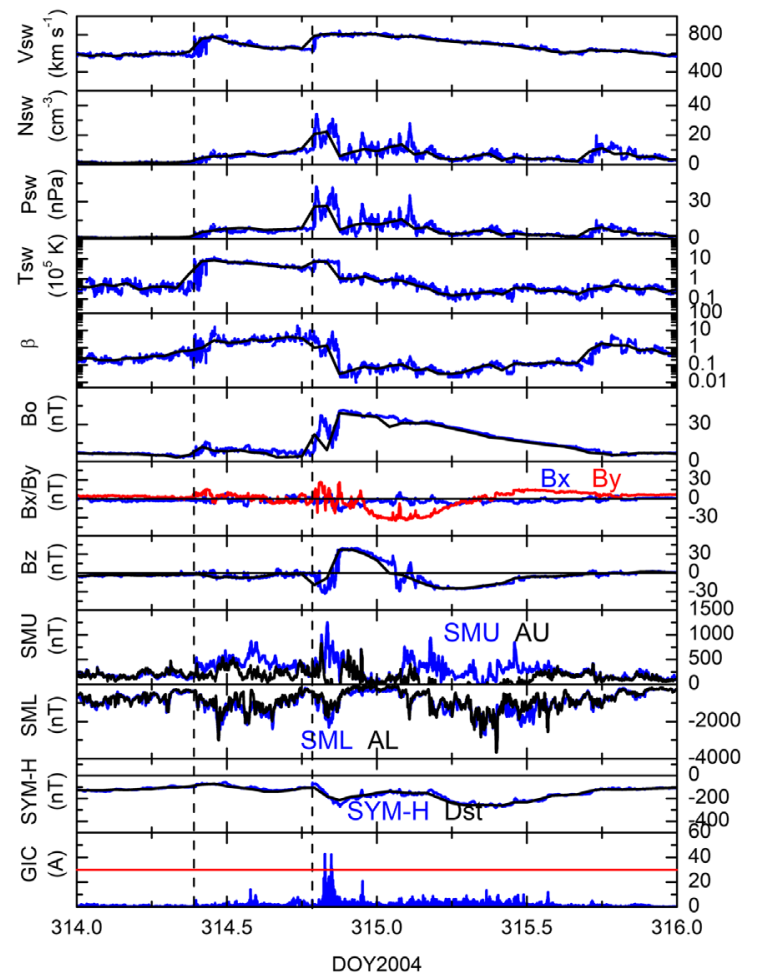

Fig. A33. Fig. A33. Events on day 314 (9 November) 2004. Same format as in Figure A1. The horizontal red line indicates the GIC = 30 A level.

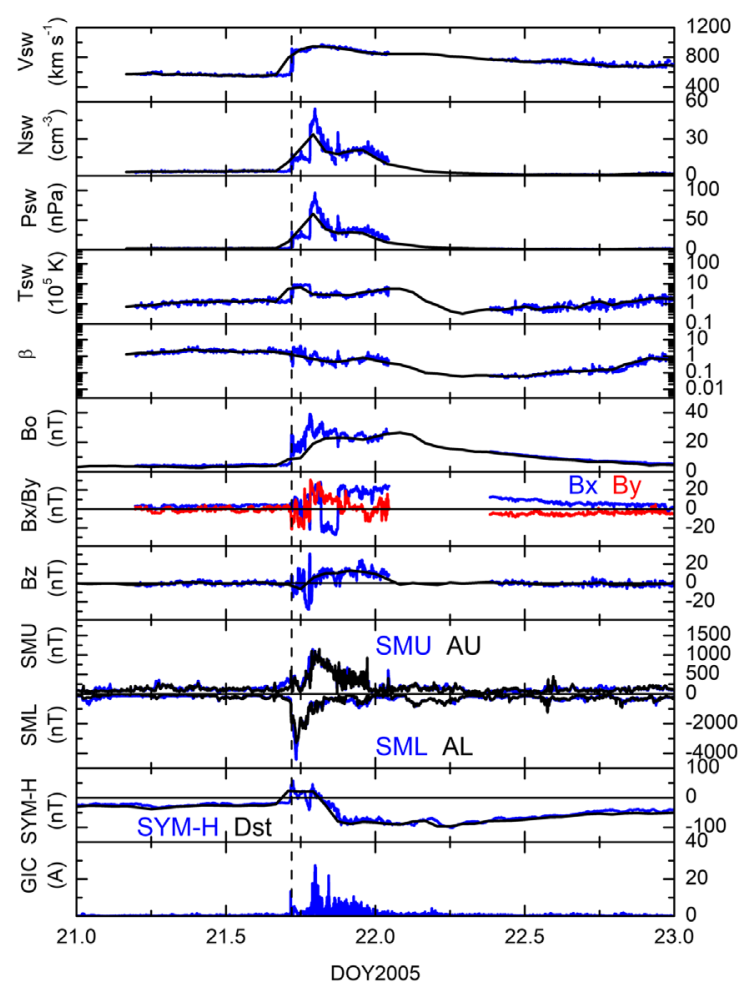

Fig. A34. Events on day 21 (21 January) 2005. Same format as in Figure A1.

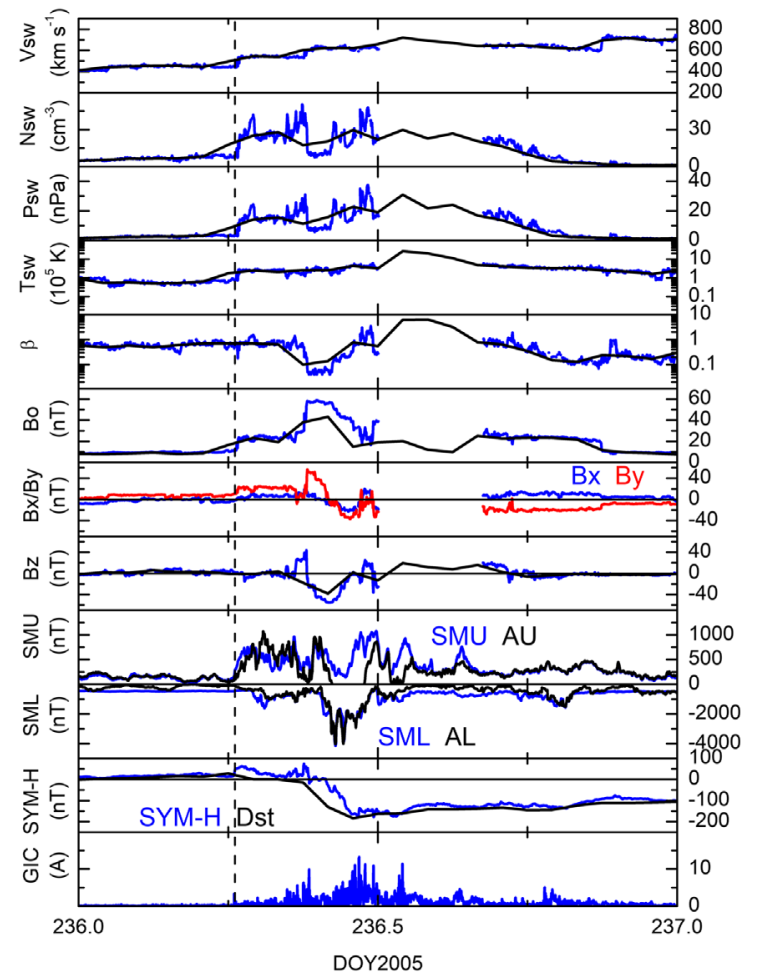

Fig. A35. Events on day 236 (24 August) 2005. Same format as in Figure A1.

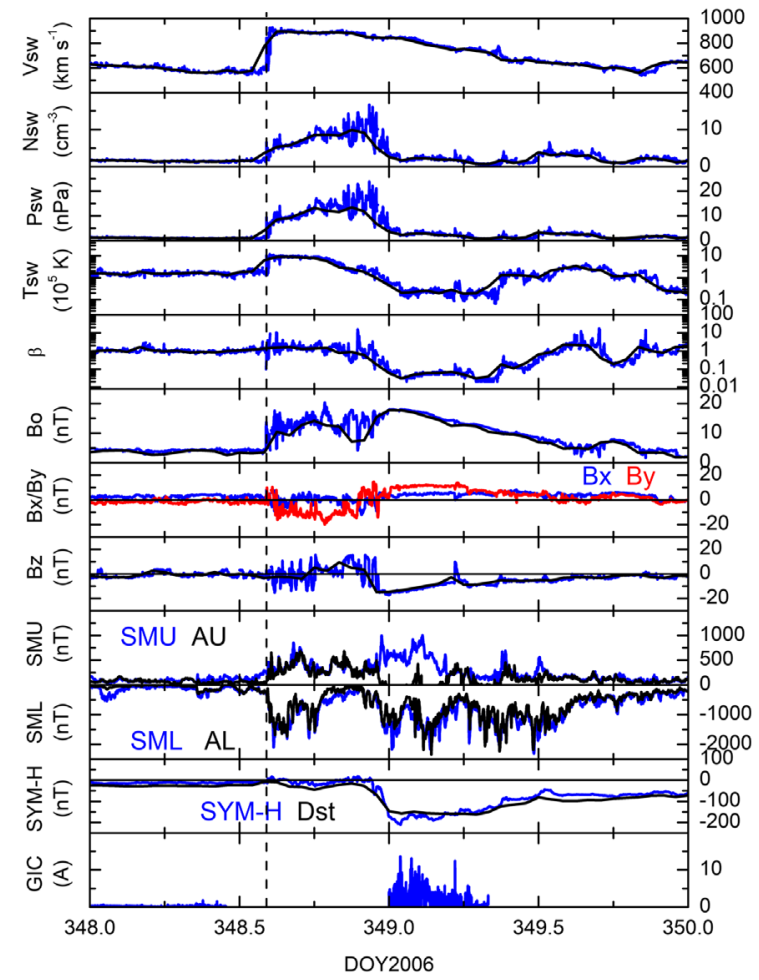

Fig. A36. Events on day 349 (15 December) 2006. Same format as in Figure A1. 


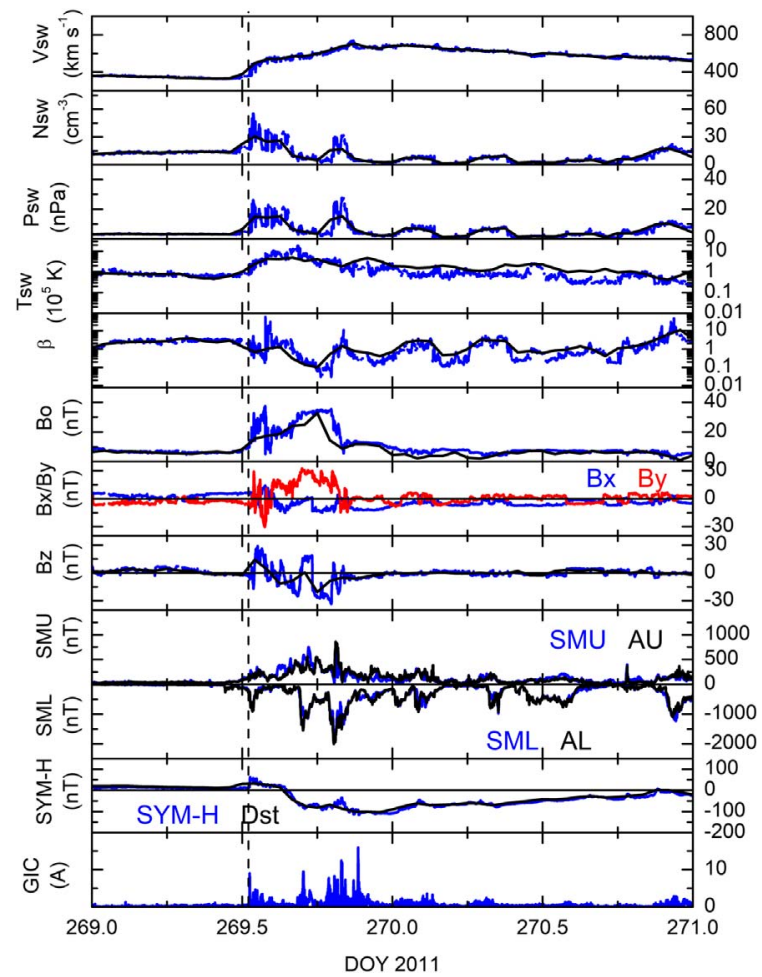

Fig. A37. Events on day 269 (26 September) 2011. Same format as in Figure A1.

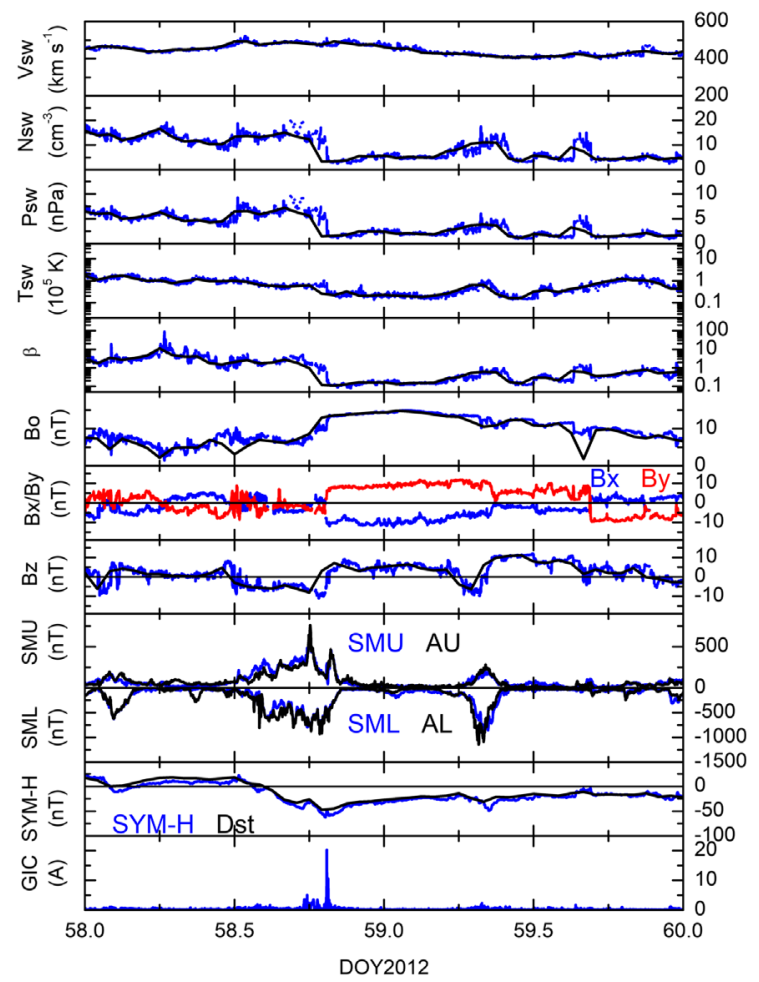

Fig. A38. Events on day 58 (27 February) 2012. Same format as in Figure A1.

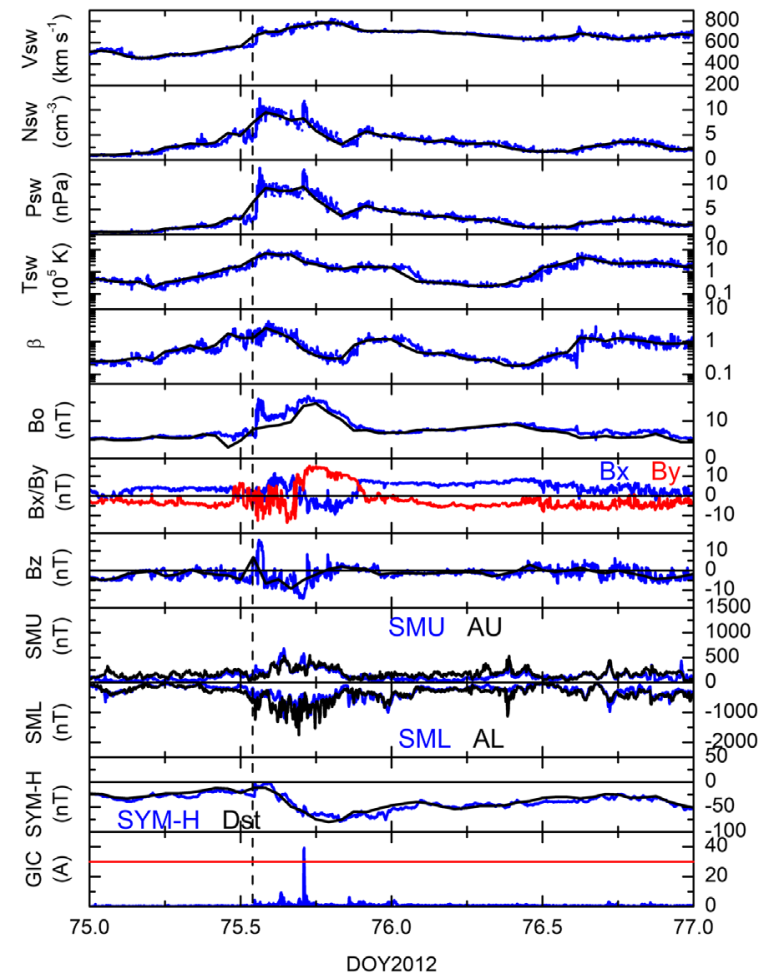

Fig. A39. Events on day 75 (15 March) 2012. Same format as in Figure A1. The horizontal red line indicates the GIC $=30$ A level.

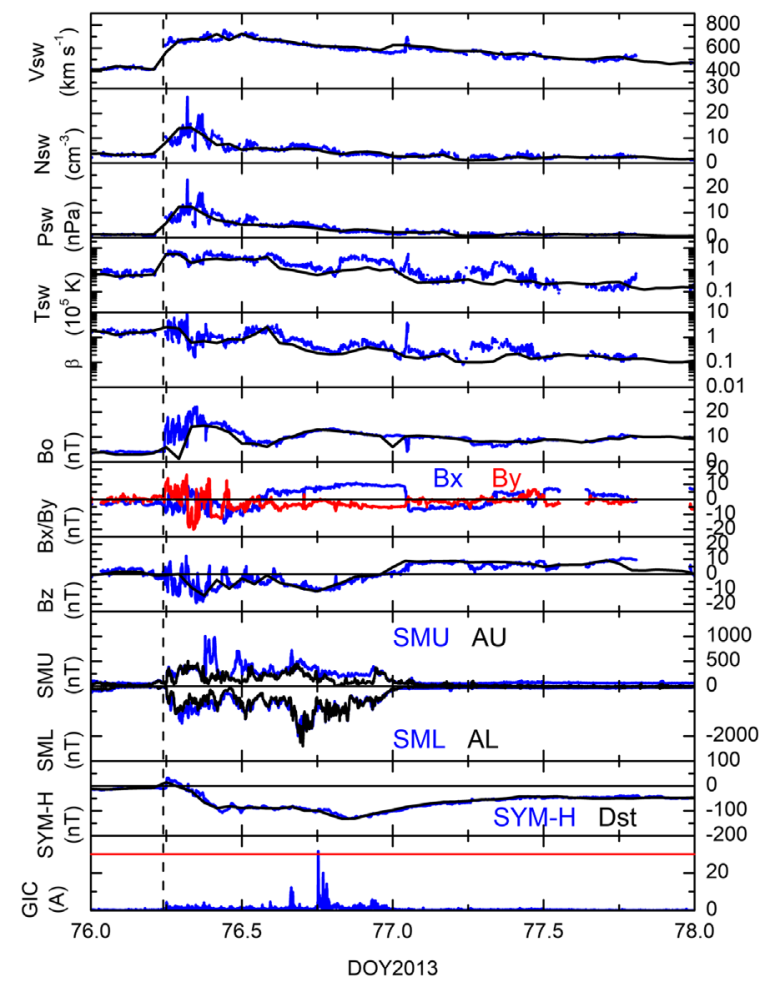

Fig. A40. Events on day 76 (17 March) 2013. Same format as in Figure A1. The horizontal red line indicates the GIC $=30$ A level. 


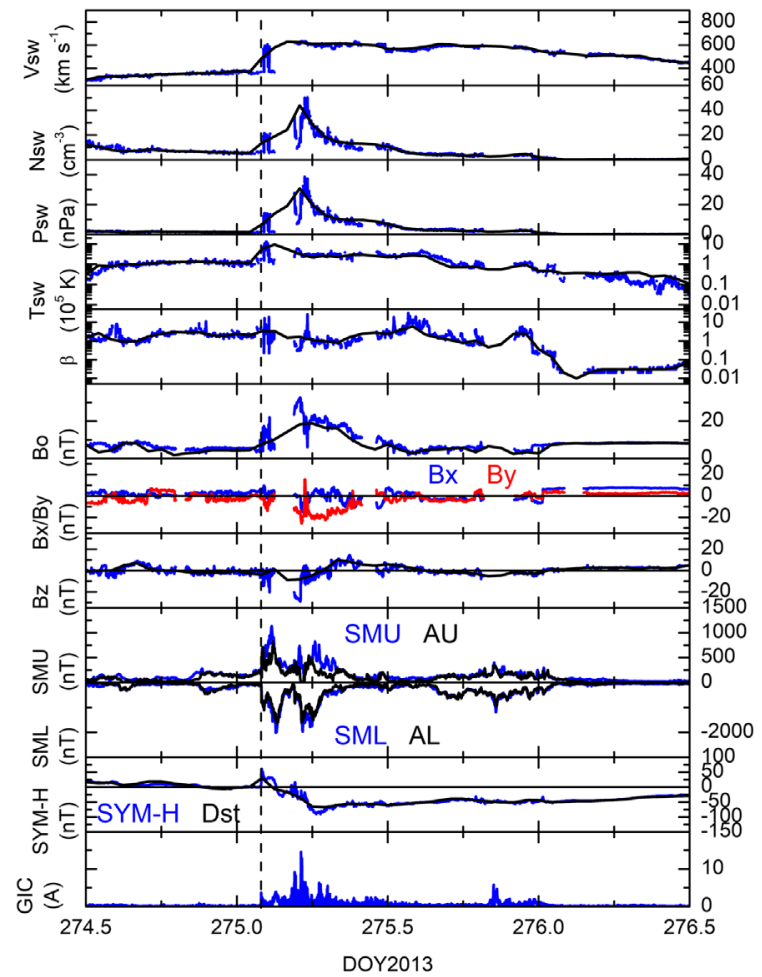

Fig. A41. Events on day 275 (2 October) 2013. Same format as in Figure A1.

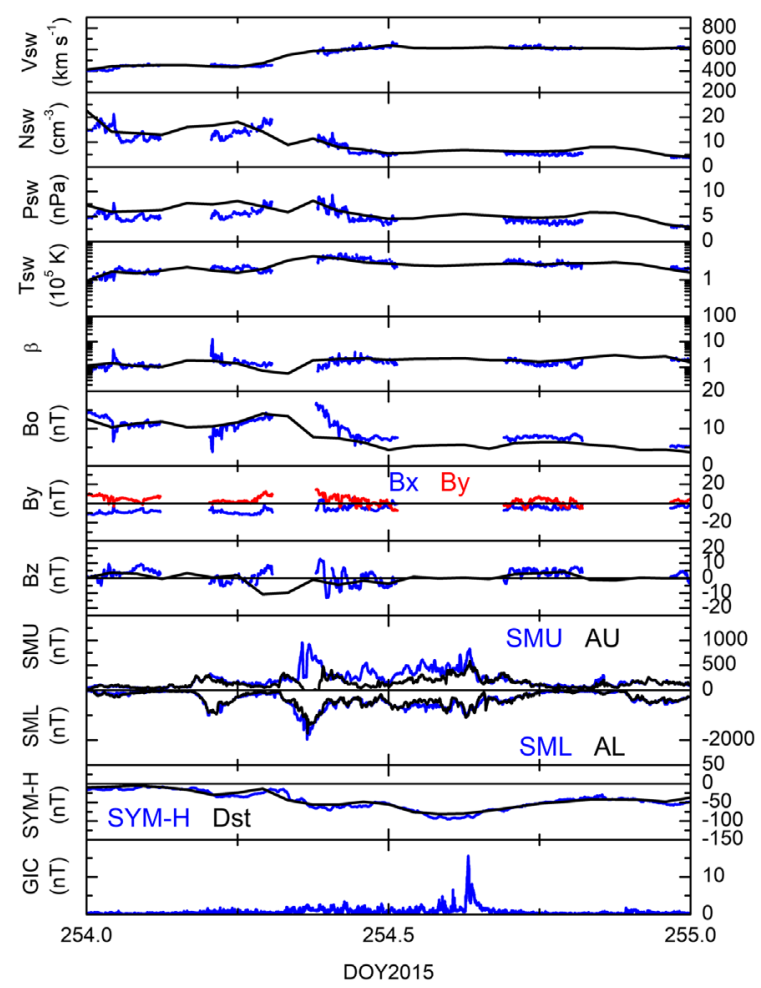

Fig. A42. Events on day 254 (11 September) 2015. Same format as in Figure A1.

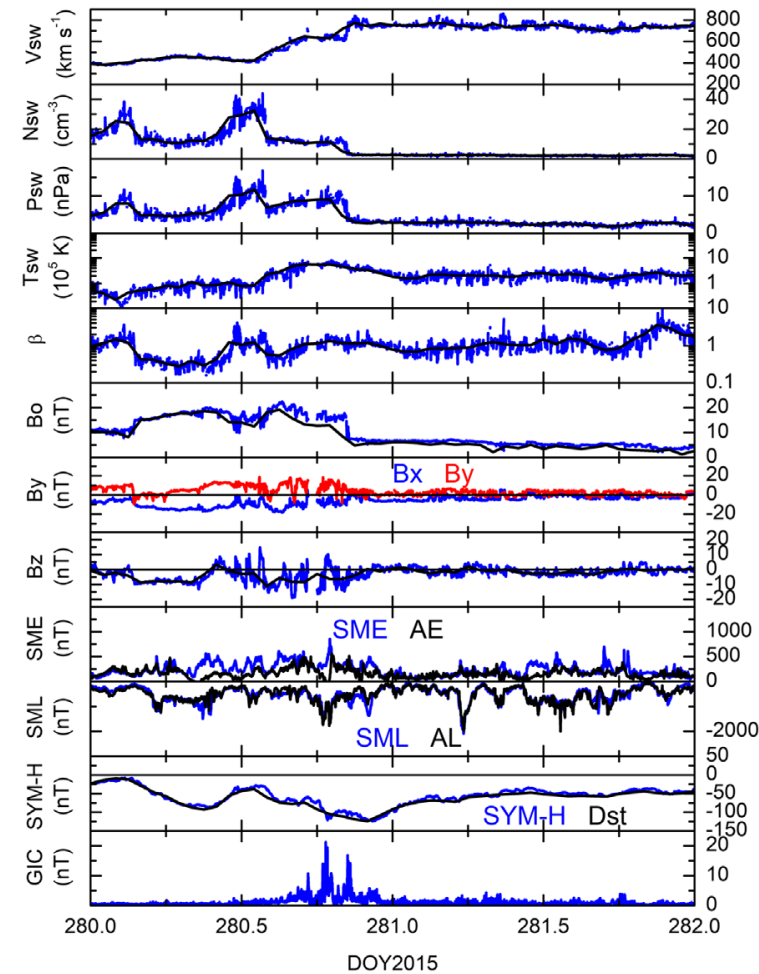

Fig. A43. Events on day 280 (7 October) 2015. Same format as in Figure A1.

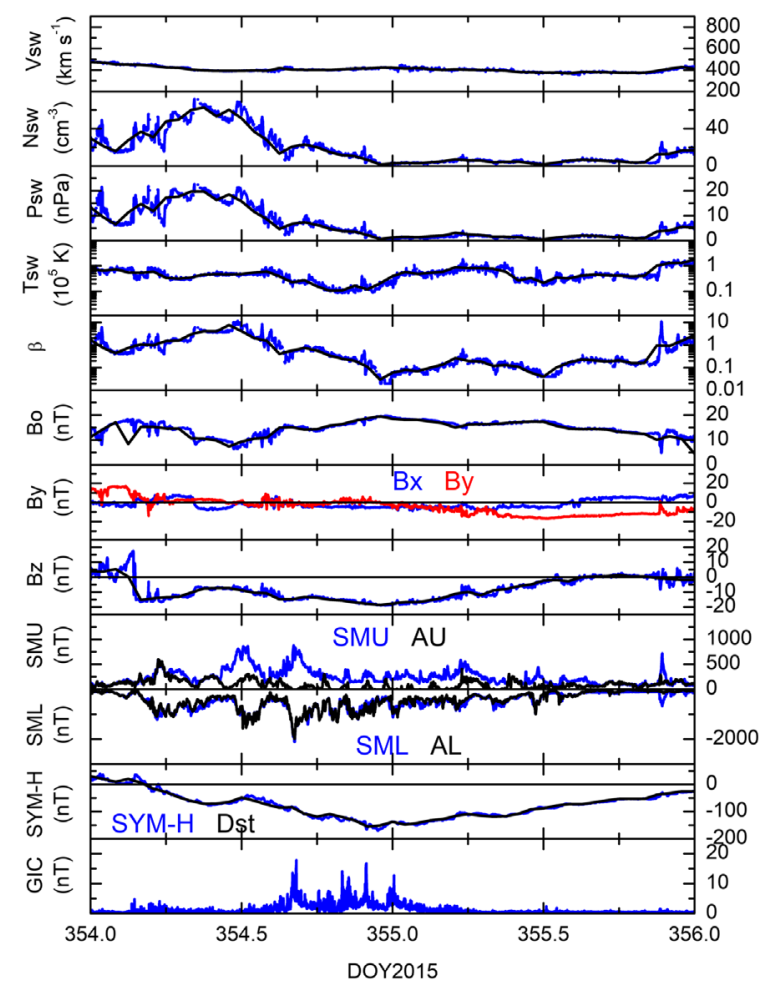

Fig. A44. Events on day 354 (20 December) 2015. Same format as in Figure A1. 


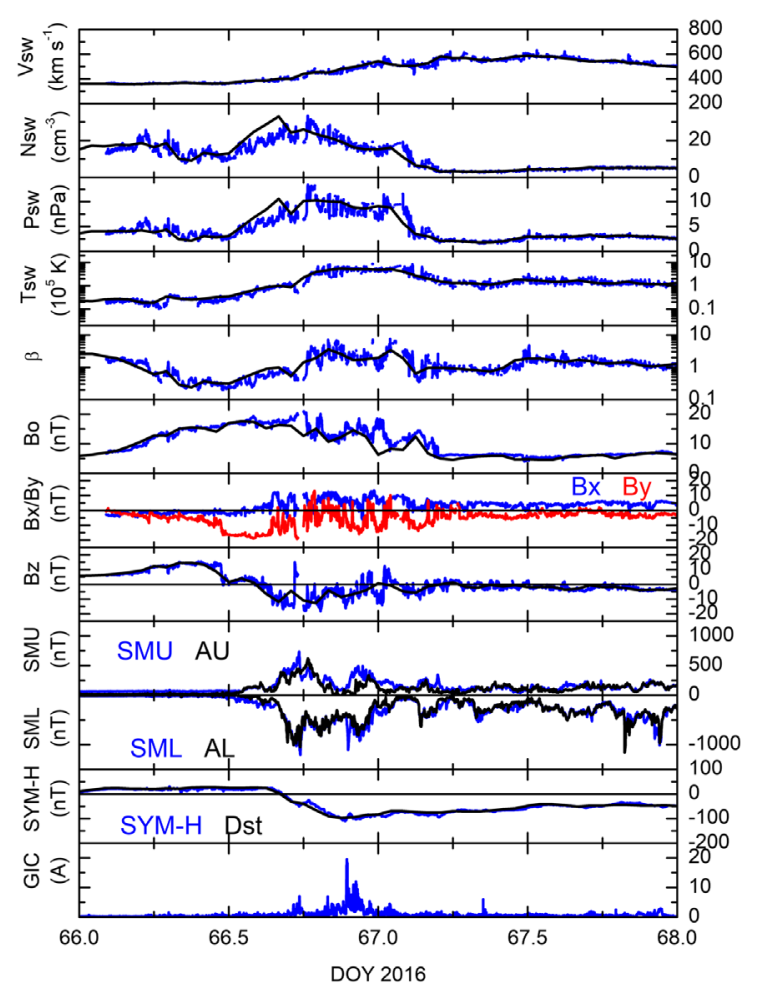

Fig. A45. Events on day 66 (6 March) 2016. Same format as in Figure A1.

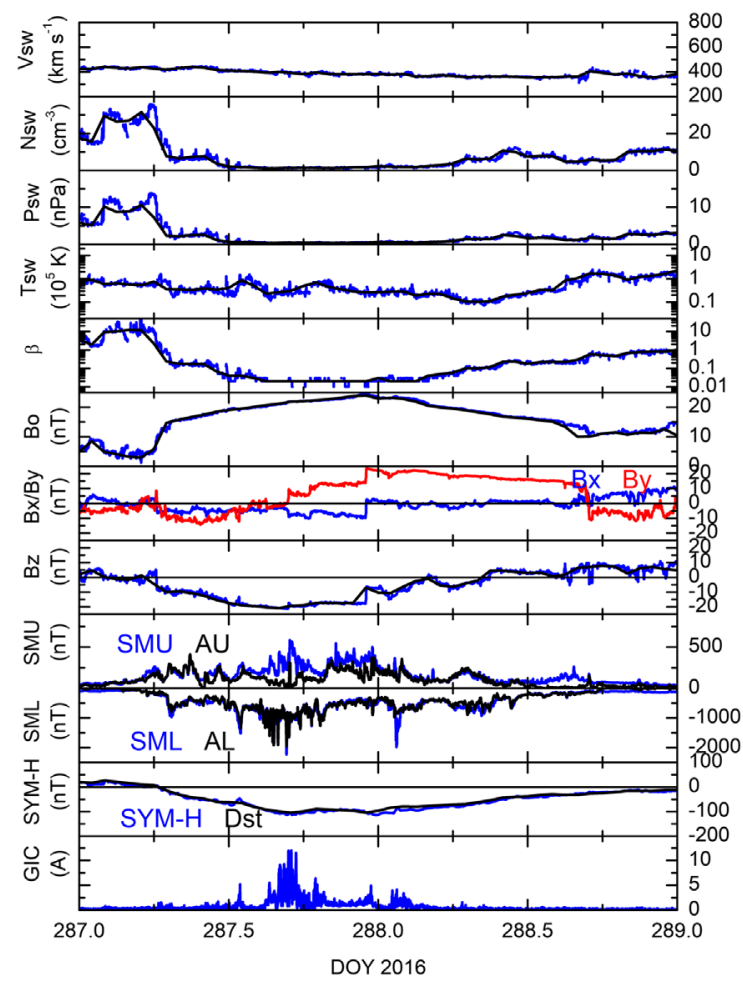

Fig. A46. Events on day 287 (13 October) 2016. Same format as in Figure A1.

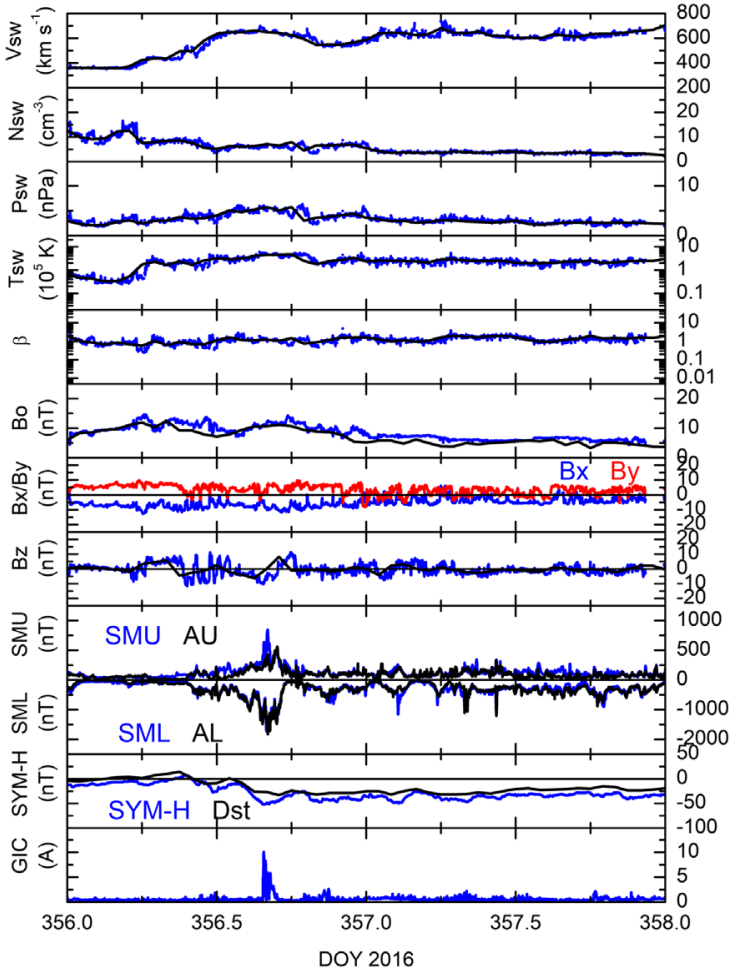

Fig. A47. Events on day 356 (21 December) 2016. Same format as in Figure A1.

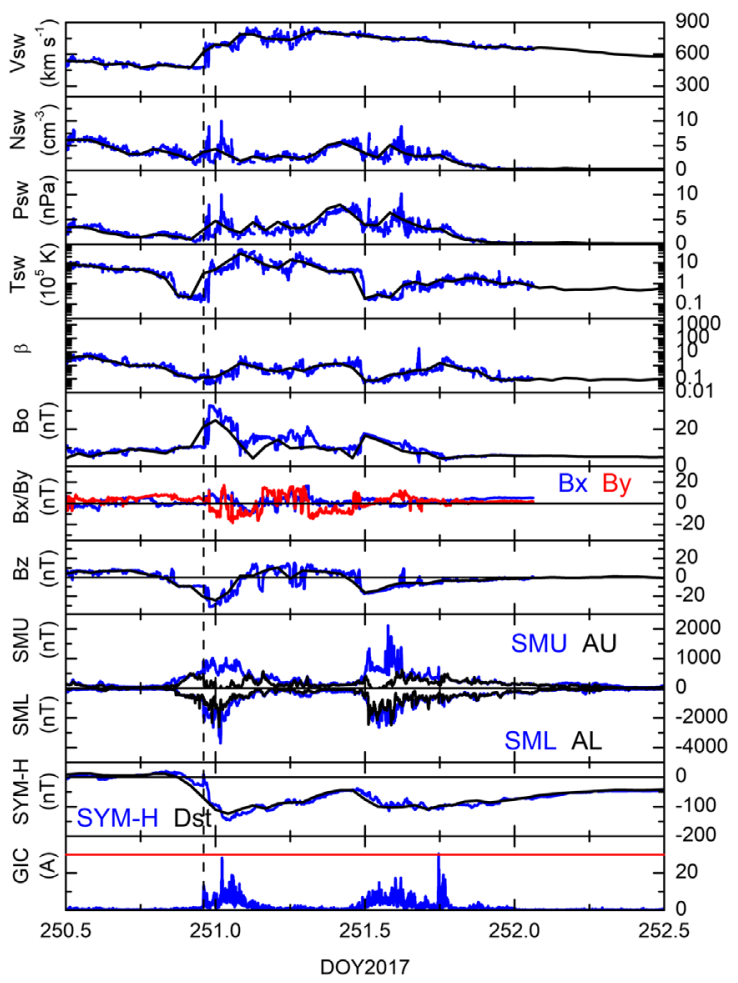

Fig. A48. Events on day 251 (8 September) 2017. Same format as in Figure A1. The horizontal red line indicates the GIC $=30 \mathrm{~A}$ level. 
any obvious substorm spike. It was associated with an IMF Bsto-Bn turning at a solar wind ram pressure decrease caused by a fast reverse shock.

Day 354 (20 December), 2015 (Fig. A44). A slow Vsw $\sim 430 \mathrm{~km} \mathrm{~s}^{-1}$ ICME MC event. Pileup region Bs and MC Bs created a double dip storm with SYM-H $=-77 \mathrm{nT}$ and $-170 \mathrm{nT}$ intensities. The pileup region Bs created the first storm dip and the slow MC Bs the second dip. There is no shock. There are no intense GICs in the first dip storm. Although the MC contains a constant Bs field here is a lot of auroral (SML) activity throughout the second dip storm. A double peak GIC of 14 and $18 \mathrm{~A}$ intensities is associated with an intense substorm of $S M L=-2106 \mathrm{nT}$. There are 3 more GIC clusters of smaller intensities with peak GICs $>10$ A that appear to be associated with smaller amplitude substorms.

Day 66 (6 March), 2016 (Fig. A45). A slow speed stream/ HSS (Vsw $\sim 590 \mathrm{~km} \mathrm{~s}^{-1}$ ) interaction created a CIR, and the Bs in the CIR caused a SYM-H = $-110 \mathrm{nT}$ storm. There was no shock. Within the CIR are Bz fluctuations superposed on a constant Bs biased interval, the latter of which caused the storm main phase. There is large geomagnetic activity up to $S M L=$ -1207 nT but no associated GICs above study threshold. A 20 A GIC event was caused by a $S M L=-1105 \mathrm{nT}$ short duration substorm. This SME spike was not detected in the $A L$ index. This GIC occurred at storm maximum.

Day 287 (Oct 13), 2016 (Fig. A46). A low speed MC of Vsw $\sim 410 \mathrm{~km} \mathrm{~s}^{-1}$ with peak magnetic field magnitude of $\sim 24 \mathrm{nT}$ and peak Bs of $\sim 21 \mathrm{nT}$ caused a $S Y M-H=-114 \mathrm{nT}$ magnetic storm. There is a large cluster of GICs with peak values reaching 12 A occurring near the storm peak. The GICs were associated with large substorms which have a peak intensity of $S M L=-2230 \mathrm{nT}$. One substorm of $S M L=-1323 \mathrm{nT}$ intensity is correlated with a GIC spike of 12 A intensity.

Day 356 (21 December), 2016 (Fig. A47). A $\mathrm{Vsw} \sim 650 \mathrm{~km} \mathrm{~s}^{-1} \mathrm{HSS}$ Bs created a weak magnetic storm of $S Y M-H=-52 \mathrm{nT}$. A small long duration Bs (with oscillations) caused a cluster of high frequency substorms with a delayed peak intensity of $S M L=-1721 \mathrm{nT}$. The peak of the substorm is associated with a $\sim 10$ A GIC cluster. In the cluster there is one event with magnitude greater than $10 \mathrm{~A}$.

Days 251 (8 September), 2017 (Fig. A48). A double dip magnetic storm of $S Y M-H=-146 \mathrm{nT}$ and $-115 \mathrm{nT}$ were generated by the upstream shock/sheath Bs and the trailing MC Bs, respectively. The shock compresses pre-existing Bs leading to a storm of $-146 \mathrm{nT}$. The shock at 2303 UT (0203 LT) causes a GIC below the study threshold. At the time of the shock Mäntsälä was in the midnight-dawn sector. A cluster of substorms occurred in the first storm main phase. A maximum intensity GIC of $28 \mathrm{~A}$ is correlated with a short duration $S M L=$ $-3712 \mathrm{nT}$ supersubstorm. There is a cluster of intense substorms/SSSs with amplitudes up to SML $=-2511 \mathrm{nT}$ and GICs up to $18 \mathrm{~A}$ intensity associated with the second storm main phase. There are no obvious 1-to-1 relationships between GIC events and substorms. The largest GIC of this interval occurred in the storm recovery phase, had an intensity of $30 \mathrm{~A}$ and cannot be associated with any large substorm or any solar wind feature.

Cite this article as: Tsurutani BT \& Hajra R 2021. The Interplanetary and Magnetospheric causes of Geomagnetically Induced Currents (GICs) > 10 A in the Mäntsälä Finland Pipeline: 1999 through 2019. J. Space Weather Space Clim. 11, 23. https://doi.org/10.1051/swsc/ 2021001. 